Portland State University

PDXScholar

$1-1-2011$

\title{
Now Accepting Applications Online: An Examination of Privacy Concerns, Explanations, and Control in Applicant Reactions to Internet-Based Selection Procedures
}

Clayton Alan Yonce

Portland State University

Follow this and additional works at: https://pdxscholar.library.pdx.edu/open_access_etds Let us know how access to this document benefits you.

\section{Recommended Citation}

Yonce, Clayton Alan, "Now Accepting Applications Online: An Examination of Privacy Concerns, Explanations, and Control in Applicant Reactions to Internet-Based Selection Procedures" (2011). Dissertations and Theses. Paper 697.

https://doi.org/10.15760/etd.697

This Dissertation is brought to you for free and open access. It has been accepted for inclusion in Dissertations and Theses by an authorized administrator of PDXScholar. Please contact us if we can make this document more accessible: pdxscholar@pdx.edu. 
Now Accepting Applications Online: An Examination of Privacy Concerns, Explanations, and Control in Applicant Reactions to Internet-Based Selection Procedures

by

Clayton Alan Yonce

A dissertation submitted in partial fulfillment of the requirements for the degree of

Doctor of Philosophy

in

Applied Psychology

Dissertation Committee Members:

Donald Truxillo, Chair

Talya Bauer

Jeanne Enders

Liu-Qin Yang

Peter Collier

Portland State University

(C)2011 


\begin{abstract}
This dissertation explores applicant reactions to Internet-based selection procedures in order to advance theory and practice related to the use modern employee selection tools. Previous authors have explored this topic area (e.g., Harris et al., 2003). However, this dissertation goes beyond previous research by proposing and testing a model that incorporates the measurement of multiple constructs that are highly relevant to organizations when utilizing Internet-based selection procedures. Such constructs include privacy concerns, explanations, control, fairness perceptions, litigation intentions, organizational intentions, and test-taking motivation. Current organizational justice theory, previous findings from studies on applicant reactions to selection procedures, and research on Internet privacy concerns provided the foundation on which this research is based. This dissertation also pulls from theory in the legal, information sciences, and psychology literatures. A model of applicant reactions that included privacy concerns and multiple outcomes relevant to organizations was proposed. Hypotheses examining this model were tested via a high-fidelity laboratory study with student participants. One-third of the participants in this study were seeking jobs at the time of participation. Findings indicated that privacy concerns are an important predictor of both proximal (i.e., fairness perceptions) and distal (i.e., organizational intentions, test-taking motivation) applicant reaction outcomes. Results also demonstrated support for a mediating role of fairness perceptions in the relationships between privacy concerns and organizational intentions as well as between privacy concerns and test-taking motivation. Providing applicants with control and explanations were found to have no moderating effect on the
\end{abstract}


relationship between privacy concerns and fairness perceptions. However, post-hoc analyses indicated that excuse explanations moderated the effect of privacy concerns on test-taking motivation. Theoretical implications of this dissertation include support for a one-factor model of organizational justice as well as a call for more integration of research from outside of industrial-organizational psychology. Additionally, areas for future research, including opportunities for improvement of study design involving timing of measures, are presented. Finally, implications for practice are discussed in regard to the possible impact of privacy concerns to large numbers of applicants participating in Internet-based selection processes, including a discussion on the importance of applicant privacy concerns to organizations and the use of multiple, inexpensive methods that may aid organizations in increasing fairness perceptions among applicants. 


\section{DEDICATION}

In honor of Eleanor F. Oitzinger Nienhaus, my maternal grandmother to whom I owe much gratitude. Though Eleanor was unable to attend college, she always stressed the importance of education and stressed the value of hard work. A salutatorian in high school, Eleanor played the saxophone, violin, and spoke both English and German. I can only hope that her fun-loving attitude, personality, and intelligence were instilled in me. Eleanor not only built a foundation on which higher education could be possible for me, but she made it probable. 


\section{ACKNOWLEDGEMENTS}

I would like to acknowledge my advisor and academic mentor, Donald Truxillo, for his great contributions to my education, as well as this dissertation. As an advisor and mentor, Donald was not only an understanding advocate, but also a motivating force. His attention to detail and in-depth knowledge enabled my success. Talya Bauer (along with Donald) helped me devise the early concepts of this dissertation, but perhaps more importantly, she was instrumental in teaching me how to effectively write for the research environment. Other members of my dissertation committee, including Jeanne Enders, Liu-Qin Yang, and Peter Collier provided excellent insights and invaluable constructive criticism. Their suggestions were much appreciated. Todd Bodner served as a consultant on statistical issues related to factor analysis, and I thank him for the expertise he provided. Finally, David Meier, Tony Sinclair, and Josh Carlsen served as research assistants and made this dissertation possible by assisting with data collection.

No list of acknowledgements would be complete, however, without mentioning the friends and family who provided support. My parents, Frank and Linda Yonce, have supported me every day of my life, and made it possible to continue my education. They always told me that I could accomplish anything. My sister, Lauren Yonce, has also provided much encouragement along the way. My friends and coworkers, including Scott Rockwell, Laura Estes, Lisa Stephany, Laura Gallaher, Adam and Larissa Dalton, Sabrina Kalish, Gordy Degear, David Penn, Michelle Fierle, Jodi Farbstein, and many others not listed here have also supported me throughout my journey. I would like to acknowledge their willingness to encourage me through the toughest of challenges. 
TABLE OF CONTENTS

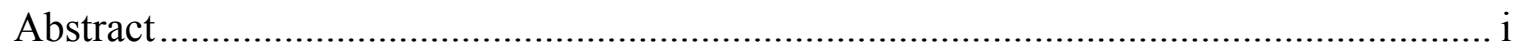

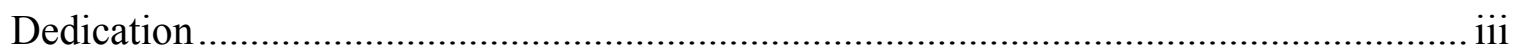

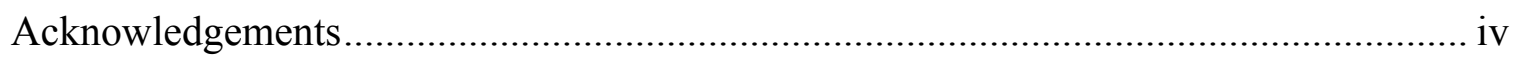

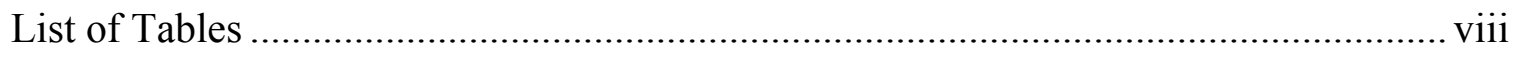

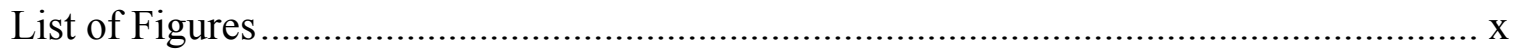

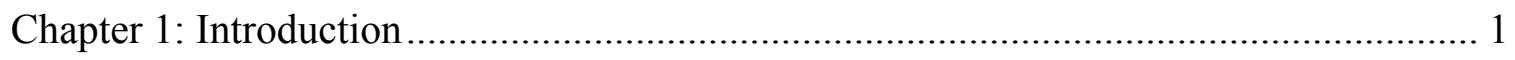

Chapter 2: The Organizational Justice Perspective …………........................................ 6

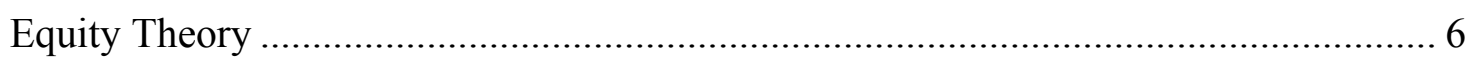

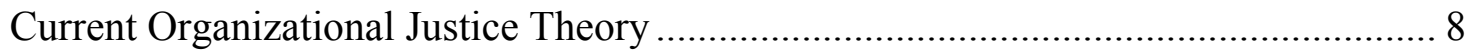

Summary of Current Organizational Justice Theory .................................................. 12

Extensions of Organizational Justice Theory ……………...................................... 12

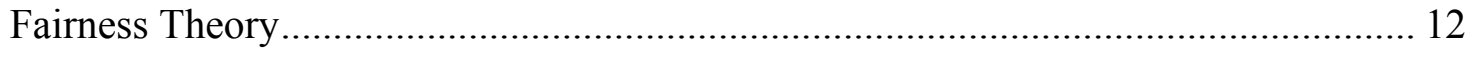

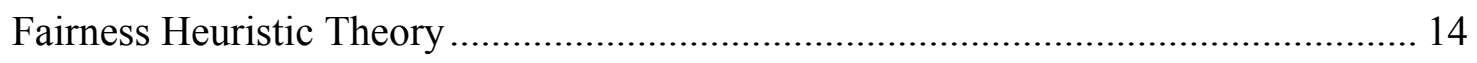

Newer Conceptualizations of Organizational Justice ……......................................... 15

Summary of Organizational Justice Approaches...................................................... 17

Chapter 3: The Study of Applicant Reactions ............................................................ 18

Historical Approaches to Applicant Reactions Research ………............................... 18

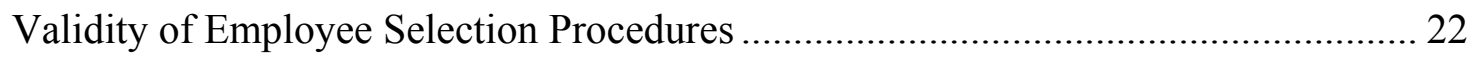

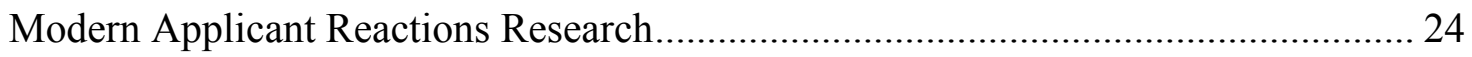

Applicant Reactions and Organizational Justice....................................................... 24

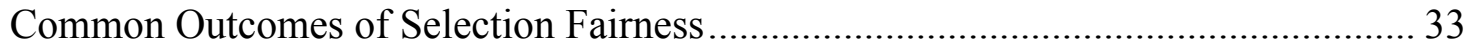


Internet-based Selection Procedures ................................................................... 42

Chapter 4: Explanations Provided to Applicants ......................................................... 45

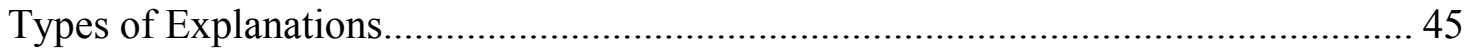

Explanation Provision and Adequacy ................................................................. 47

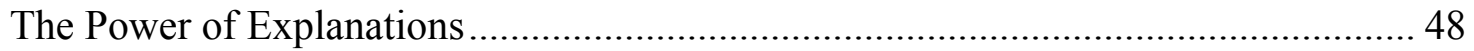

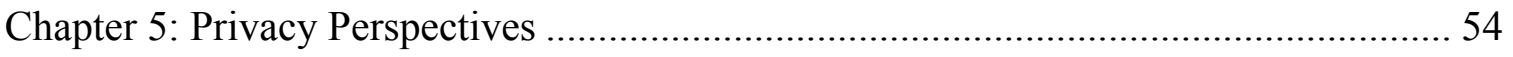

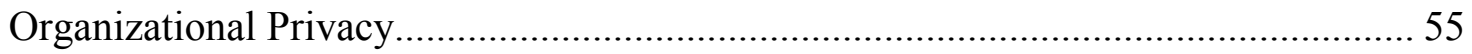

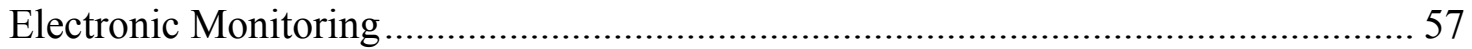

Privacy in Personnel Selection..................................................................... 58

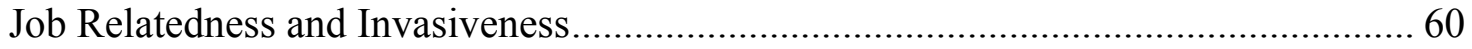

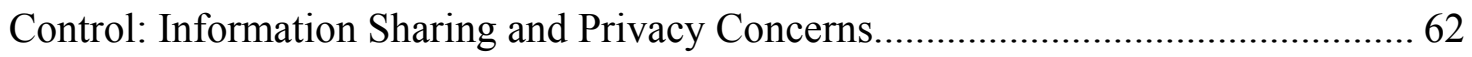

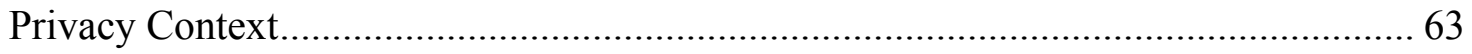

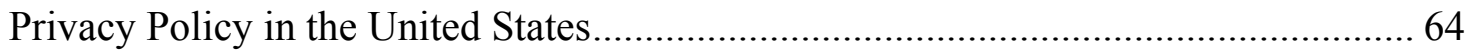

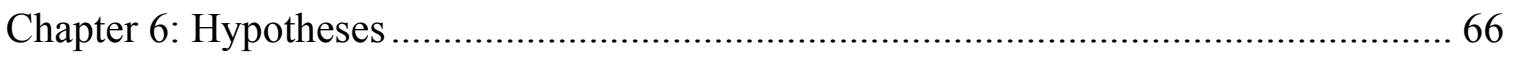

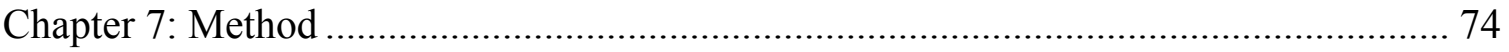

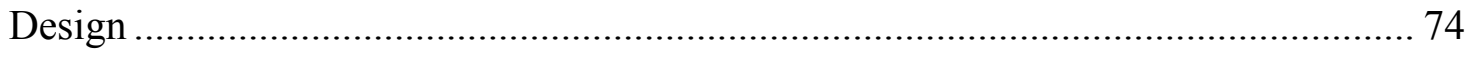

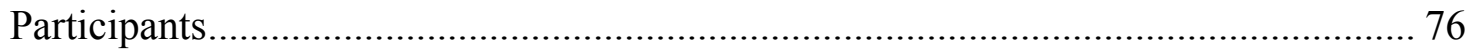

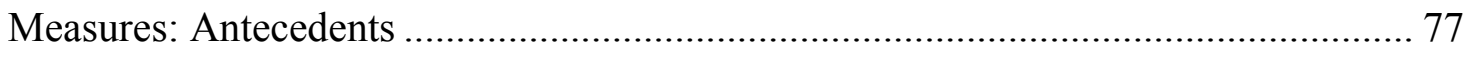

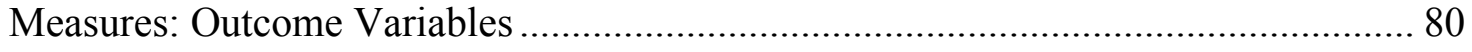

Measures: Potential Control Variables .............................................................. 82

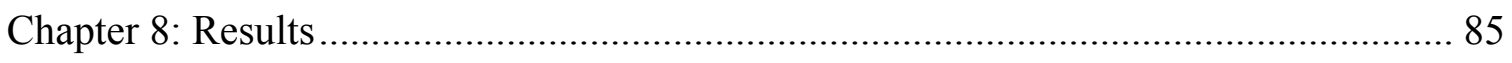

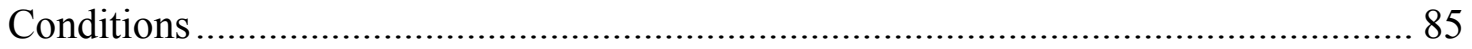




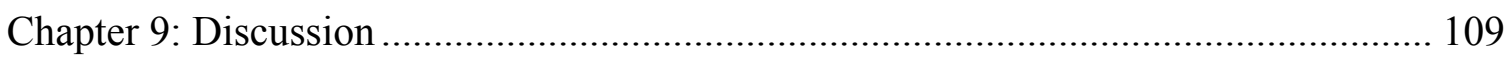

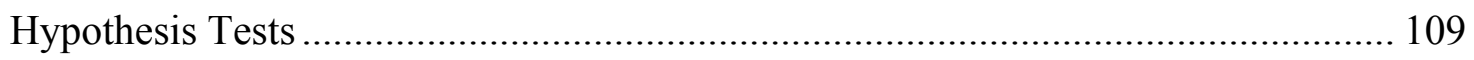

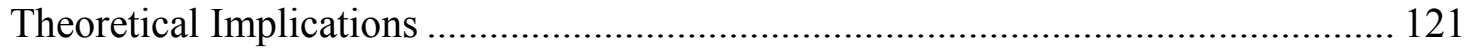

Future Research ........................................................................................ 123

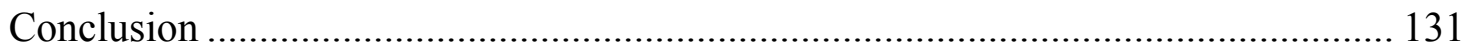

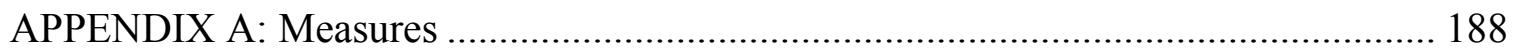

APPENDIX B: Recruitment Form ................................................................ 198

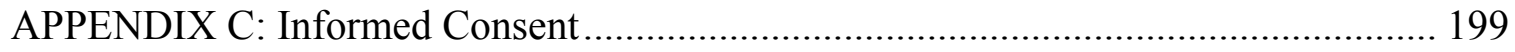

APPENDIX D: Letter to Participant.................................................................... 200

APPENDIX E: Employment Application and Test.................................................. 201

APPENDIX F: Excuse Explanation Condition ..................................................... 209

APPENDIX G: Privacy Justification Explanation Condition................................... 210

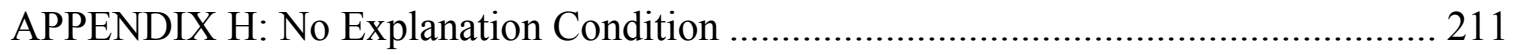

APPENDIX I: Control Over Information Condition ............................................. 212

APPENDIX J: No Control Over Information Condition ....................................... 213

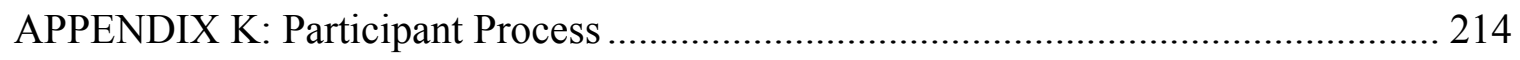

APPENDIX L: Decision Letter - Post-Test Excuse Explanation Condition ................. 215 APPENDIX M: Decision Letter - Post-Test Privacy Justification Explanation Condition 216

APPENDIX N: Decision Letter - Post-Test No Explanation Condition....................... 217

APPENDIX O: Thank You/Debrief Letter........................................................... 218

APPENDIX P: Prize Award ............................................................................. 219 
List of Tables

Table 1. Table of Experimental Conditions.

Table 2. Listing of Measures

Table 3. Means, Standard Deviations, Correlations, and Internal Consistency Estimates

for Pilot Study Variables.

Table 4. Summary of ANOVA: Differences between Central Florida Sample and Pacific

Northwest Sample

Table 5. Summary of ANOVA: Differences between White/Caucasian and Non-

White/Non-Caucasian Participants

Table 6. Means, Standard Deviations, and Intercorrelations of Study Control Variables

and Study Scales

Table 7. Means, Standard Deviations and Intercorrelations for Study Pre-Test and Post-

Test Measures 143

Table 8. Summary of Hierarchical Regression Results for Hypotheses 1-3 ................ 146

Table 9. Summary of Hierarchical Regression Results for Research Question 1 ........ 148

Table 10. Summary of Hierarchical Regression Results for Hypothesis 4a................. 149

Table 11. Summary of Hierarchical Regression Results for Hypothesis 4b ................ 150

Table 12. Summary of Hierarchical Regression Results for Hypothesis 4c................. 151

Table 13. Mediation Tests: Litigation Intentions..................................................... 152

Table 14. Mediation Tests: Organizational Intentions............................................ 153

Table 15. Mediation Tests: Test-Taking Motivation ............................................ 154

Table 16. Summary of Hierarchical Regression Results for Research Question 2 ........ 155 
Table 17. Pre-Test and Post-Test Means by Study Condition for Distal Outcome

Variables 156 
List of Figures

Figure 1. Model of Applicants’ Reactions to Employment Selection Systems .............. 157

Figure 2. Hypothesized Overall Model of Applicant Reactions to Privacy Concerns ... 158

Figure 3. Hypothesized (Revised) Overall Model of Applicant Reactions to Privacy

Concerns

Figure 4. Hypothesized Relationship between Privacy Concerns and Explanations in

Predicting Fairness Perceptions ............................................................................ 160

Figure 5. Hypothesized Relationship between Privacy Concerns and Control in Predicting

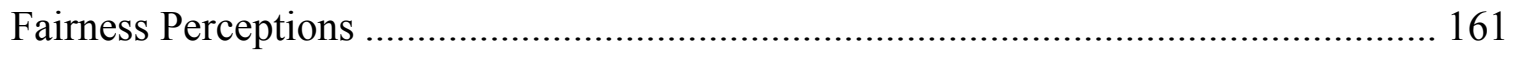

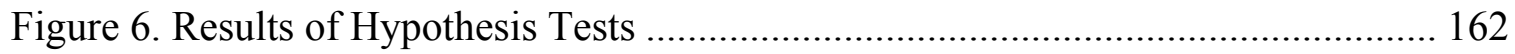

Figure 7. Confirmatory Factor Model for Test-Taking Motivation.............................. 163

Figure 8. Confirmatory Factor Model for Organizational Intentions .......................... 164

Figure 9. Confirmatory Factor Model for Litigation Intentions ................................ 165

Figure 10. Initial Confirmatory Factor Model for Overall Fairness Perceptions ........... 166

Figure 11. Final Confirmatory Factor Model for Overall Fairness Perceptions............. 167

Figure 12. Confirmatory Factor Model for Dimensions of the Concerns for Information

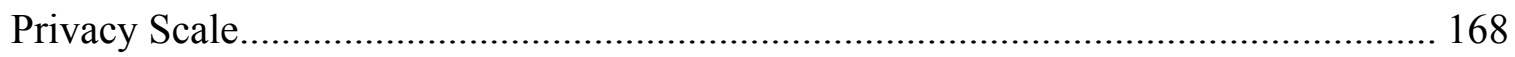

Figure 13. Relationship between Privacy Concerns and Explanations in Predicting Test-

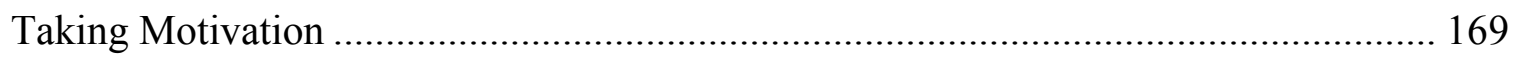




\section{Chapter 1:}

Introduction

New technology provides organizations with the opportunity to electronically automate many of their Human Resource (HR) functions. Employee files have been moved out of the file drawer and onto mainframe servers, performance appraisals that were once tangible forms are now completed on organizational intranets, and employment applications that were once paper-based are now completed via the Internet or computer kiosk.

Internet-based selection tools are increasingly common following the emergence of numerous organizations which provide electronic hiring management services to large organizations (Scarborough \& Somers, 2006), many of whom accept thousands of applications for various positions worldwide every day (Handler \& Hunt, 2003). As a result, many job-seekers are required to submit personal information electronically via Internet-based selection tools. More individuals than ever have their highly-sensitive personal information captured, transmitted, and stored electronically (Dinev \& Hart, 2004), and job-seekers may approach such electronic processes with great acceptance, great apprehension, or both.

When considering what it means to transmit personal data over the Internet, one must consider the issue of privacy. Privacy issues have been studied from the theoretical orientations of several different fields, including communications (e.g., Millberg, Smith, \& Burke, 2000), law (e.g., Taylor, 2008), popular culture (e.g., Russell, 1999), information sciences, (e.g., Zhang \& Zhao, 2008), consumer behavior (e.g., Lauer \& 
Deng, 2007), and psychology (e.g., Stone \& Stone, 1990). In the past several years, privacy issues have garnered significant attention outside of academic fields and in the mainstream media. News websites such as MSNBC.com feature sections devoted to privacy issues in the information age. In fact, in MSNBC.com's "Privacy Lost" article series, readers can learn about what is referred to as real-life privacy "nightmares" in which "seemingly innocuous actions" had "devastating consequences" due to the use of personal information by outside entities (Sullivan, 2006). Other articles include in-depth investigations of differences in privacy regulations in the United States versus the European Union (Sullivan, 2006), and surveys regarding peoples' views on losing privacy ("Is your privacy important?," 2006). Privacy has also been a concern as the use of social media tools, such as Facebook.com, has increased. In fact, the Electronic Privacy Information Center, a public interest research center, stated that "Facebook has several features with a significant impact on privacy and security of personal information. These features raise issues of data collection, retention, distribution, and control," (2011). As such, media has raised an awareness of possible concerns related to privacy. Issues in Internet-based selection are far-reaching, as they are not just an issue of importance to the United States, but also to the international community. The popular media's attention to this issue continues to grow due to the fact that the transmission of personal data across networks and between entities is becoming more common as multinational organizations seek to market and sell their goods across borders (Bellman, Johnson, Kobrin, \& Lohse, 2004; Dinev \& Hart, 2004). As a result, many multi-national companies receive large amounts of personal data, leaving consumers and scholars with 
questions regarding the protection of such data. As companies expand globally, they also seek to place personnel in strategic global locations. This often results in the implementation of Internet-based selection tools that capture applicants' personal information (Handler \& Hunt, 2003). From both the consumer and job applicant view points, there remain questions regarding the privacy of the personal data transmitted over such networks.

Whether applying for a job online, purchasing a shirt from a clothing website, or participating in web-based social media tools like Facebook, end-users (the applicant or the consumer) submit some type of personal information over an Internet-based network. In question here are the reactions that individuals have who must apply for jobs using the Internet. A central theme regarding job applicants' concerns with online selection tools focuses on the privacy of the personal information shared. Several authors have studied the invasiveness of particular selection procedures/ tools (Stone-Romero, Stone, \& Hyatt, 2003), justice perceptions as they relate to electronic performance monitoring (Alge, 2001; Ambrose \& Alder, 2000), general applicant reactions toward Internet-based selection procedures (Bauer et al., 2006; Sinar \& Reynolds, 2001), and attitudes toward privacy and using the Internet (Dinev \& Hart, 2004; Jensen, Potts, \& Jensen, 2005). Still, no author has presented a model of applicant reactions to Internet-based selection procedures that both incorporates applicant privacy concerns and uses concepts from organizational justice theory to present practitioners with manipulations that can be used in real-world settings. Such manipulations, like control and explanations, can be used in real-world settings to create more positive applicant reactions. 
First, this dissertation built on the work of Bauer et al. (2006) and Harris, Van Hoye, and Lievens (2003) by investigating applicant privacy concerns from an organizational justice perspective. Specifically, this dissertation examined the effect that privacy concerns have on applicants' reactions to the selection process itself and the organization. Consistent with the themes introduced by the organizational justice perspective (Bies \& Moag, 1986), explanations and control were manipulated in an investigation of applicant privacy concerns. These two manipulations are supported by previous research related to fairness perceptions and privacy concerns.

Second, the use of a laboratory setting to conduct this study provided for greater control over the manipulations, random assignment of participants to conditions, and allowed for the measurement of variables that are more difficult to measure in real-world settings, such as litigation intentions (see Chapter 3: The Study of Applicant Reactions). Though laboratory setting can limit the generalizability of study results, the highly realistic design of the procedure used in this study, as well as participant demographics (see Chapter 8: Results), helped to mitigate this concern. Furthermore, the measurement of outcomes often restricted from measurement in real-world contexts (i.e., litigation intentions) provides a solid connection between manipulations and outcomes that are highly relevant to organizations.

Third, this dissertation sought to advance both theory and practice as it proposed and tested hypotheses related to a model of applicant reactions that incorporated applicant privacy concerns and moderating variables including explanations and applicant control. The results of this study provide a more comprehensive view of applicant reactions 
theory and recommendations to aid practitioners in eliciting positive applicant reactions from candidates who apply for positions via Internet-based selection procedures.

Fourth, this dissertation utilized findings and knowledge gained from multiple disciplines. Such "interdisciplinarity" has allowed bodies of research to rapidly advance and mature (Thagard, 2010). This study integrates findings from the legal, psychological, and information sciences literatures. For example, the most common approach to the study of applicant reactions stems from organizational justice theory, which has roots in the legal literature (e.g., Bies \& Moag, 1986; Thibaut \& Walker, 1975). Organizational justice is now a well-accepted approach to research in psychology, and in particular, industrial-organizational psychology (e.g., Greenberg, 1993). This dissertation also incorporated theory derived from the information sciences literature, as this literature has previously demonstrated the measurement of privacy concerns has been demonstrated in this literature (e.g., Stewart \& Segars, 2002).

In all, this study represents a significant contribution to theory and practice by building on the work of previous authors, utilizing a rigorous design, proposing and testing a model which incorporates important determinants of applicant perceptions, and taking a multi-disciplinary approach. The results obtained from this study offer researchers more insight into the study of applicant reactions and offer practitioners more insight into actions which can mitigate negative responses and increase positive applicant reactions to Internet-based selection procedures. 


\section{Chapter 2:}

The Organizational Justice Perspective

Organizational justice has received a large amount of attention from researchers in industrial-organizational psychology. As a result, organizational justice theory has been applied to many different research streams, including leadership (e.g., Pillai, Schriesheim, \& Williams, 1999), performance management (e.g., Masterson, Lewis, Golman, \& Taylor, 2000), and applicant reactions (e.g., Gilliland, 1993). To clearly understand organizational justice theory, it is important to recognize its development.

As such, I will first explore Equity Theory as it relates to the development of organizational justice theory. Next, I will describe organizational justice theory by examining the four key types of justice described by the theory (distributive justice, procedural justice, informational justice, and interpersonal justice). Then, I will examine three recent organizational justice theories which are relevant to this study: fairness theory, fairness heuristic theory, and the event mediation approach. Finally, I will summarize the key outcomes associated with organizational justice theory. Equity Theory

Equity theory served as the foundation upon which organizational justice theory was built. Adam's (1965) Equity Theory, which is based on social comparison, suggests that individuals evaluate "inputs" relative to "outputs". Inputs and outputs refer to individuals' respective contributions to a relationship. For example, an employee's input may be viewed as the time and effort he/she put into a work project. An output related to this input may be viewed as the monetary rewards given to the employee from his/her 
supervisor as a result of the aforementioned inputs. The subsequent evaluation of time and effort (inputs) relative to the supervisor's assignment of monetary rewards results in behavioral adjustments. In this example, a behavioral adjustment may manifest itself in the form of less time and effort put forth by the employee given that the employee views the monetary reward distributed by the supervisor as inadequate. Conversely, should the employee view the monetary reward as adequate, he/she may maintain or increase performance to ensure that subsequent monetary distributions continue.

Central to the process described above is the existence of a "referent other" which serves as a point of comparison. This is similar to social comparison theory, where individuals compare themselves to others (Festinger, 1954). Often, the referent other is a “coworker, individual in a previous situation, a hypothetical 'ideal other,' or a system referent" (Donovan, 2001, p.55). Therefore, individuals may target multiple referents for comparison and adjust behavior based on such comparisons. The referent other in the example provided may be a coworker who the employee sees as putting forth comparable effort. If the reward given to the referent other is larger than that given to the employee, the employee may adjust his/her behavior accordingly.

Although the theory states that individuals will make adjustments in behavior, there is little research that provides an explanation of how individuals choose a particular method of adjustment in response to inequity (Donovan, 2001). Rather, Adams (1965) suggests only that attempts will be made to restore equity in situations. It is believed that such equity restoration attempts often involve holding an entity accountable for his 
actions, and adjusting "inputs" to match "outputs" in terms of the relationship (Folger \& Cropanzano, 2001).

\section{Current Organizational Justice Theory}

Borne out of the general ideas presented by Adam's Equity Theory, organizational justice has emerged as a popular conceptualization of fairness perceptions in work-related contexts. Organizational justice is comprised by four main perceptions. First, distributive justice is concerned with individual perceptions of the fairness of particular outcomes associated with the process (Greenberg \& Cropanzano, 2001). Perceptions of distributive justice are akin to the reactions an individual has relative to the "outputs" described in Adam's (1965) Equity Theory. In other words, distributive justice perceptions are formulated based upon what an individual receives as a result of a procedure.

Relatedly, the second component of organizational justice theory is procedural justice. Procedural justice concerns individual perceptions of the fairness of a particular process (Thibaut \& Walker, 1975) rather than solely the outcome of that process. Unlike distributive justice, which focuses on economic benefit (i.e., comparing inputs to outputs), procedural justice emphasizes socio-emotional aspects of decisions in relation to the means by which a decision is rendered (Cropanzano \& Ambrose, 2001), as highlighted by Levanthal's (1980) six criteria. Though procedural justice was first derived from legal contexts, Levanthal later applied procedural justice to the organizational context. In his theory, Levanthal proposed six criteria under which procedures face judgment in order to be perceived as fair. These included (1) consistent 
application of procedures across people and across time, (2) lack of bias or vested interest of a third party in a particular outcome, (3) assurance of accurate information collection and use in decision-making, (4) a corrective mechanism by which to provide reparations for disparate decisions, (5) conformity to moral/ethical standards, and (6) respect for the opinions of the entities affected by decisions.

A third component of organizational justice theory has been described as “interactional justice.” However, this type of justice has since been further refined by some authors to include two distinct types of justice: interpersonal justice and informational justice. The concept of interactional justice is related to the belief that relationships supply people with both economic and socio-emotional benefits, as asserted by Cropanzano and Schminke (2001). Such benefits are of particular relevance to fairness theory and organizational justice theory because they signal "the present or future attainment of a material or socioemotional benefit" whereas "injustice signals the absence of such valued beliefs" (Folger \& Cropanzano, 2001). Bies and Moag (1986) identified interactive justice as unique and different because they believed that procedural justice did not "capture the breadth of people's concerns about a "fair process"” (Bies, 2001, p.90). Consistent with the aforementioned relationships between fairness and socioemotional benefits, interactional justice is concerned with the perceptions of fairness related to interpersonal interactions between the individual and the person or entity distributing the outcome (Bies \& Moag, 1986).

The interactions inherent to interactional justice are comprised of perceptions of interpersonal relations, such as individuals' beliefs that entities involved in rendering the 
decision treated them fairly (interpersonal justice). The interactions are also comprised of perceptions of the information provided by entities involved in decision making, such as individuals' beliefs that they were provided with adequate amounts of information about processes and outcomes (informational justice). Thus, since the introduction of interactional justice to the organizational justice perspective, some authors have refined interactional justice by asserting that it is comprised of both interpersonal justice and informational justice (Greenberg, 1993). In fact, Greenberg specified that interpersonal justice is said to capture individuals' perceptions of interpersonal treatment throughout a process. Informational justice is said to capture individuals' perceptions of the explanations offered for outcomes. Colquitt, Conlon, Wesson, Porter, and Yee (2001) confirmed that interpersonal justice and informational justice are related to different outcomes, thereby implying that interactional justice is made-up of two distinct components. Due to the Internet-based context of this study, this dissertation does not explicitly explore interpersonal justice, informational justice, or the interactional justice concept.

The debate regarding the multi-faceted nature of interactional justice has not been the sole debate in the organizational justice literature. In fact, there has been much debate regarding all of the different types of justice and their relationships with one another. This debate was motivated by individual studies that showed that each type of justice was moderately to strongly correlated with one another. (e.g., Gilliland, 1994; Kernan \& Hanges, 2002; Simons \& Roberson, 2003). Meta-analytical methods have since 
corroborated these findings (Colquitt et al., 2001). As a result, authors have questioned the distinctiveness of the justice constructs (Folger, 1987).

One assertion of the similarities among the types of justice is the monistic perspective. As described by Cropanzano and Ambrose (2001), the monistic perspective argues that perceptions of procedural justice are dependent on the outcomes distributed from the procedure. Cropanzano and Ambrose propose that individuals are concerned with processes because they are instrumental to outcomes. They state:

... when people make judgments of procedural justice, they are actually attending to their possible long-range economic or instrumental consequences. Under this view, procedural fairness can be viewed as a type of enlightened outcome fairness; procedures are evaluated relative to the (economic) consequences that they may produce. (p.124)

This view suggests a strong, cognitively salient link between procedural justice perceptions and distributive justice perceptions.

Colquitt et al. (2001) sought to address the issue of similarity between justice constructs in their meta-analysis of 183 organizational justice studies. Their findings indicated that the different types of justice contribute to the prediction of incremental variance in fairness perceptions. Colquitt et al. suggested that even though the justice constructs are correlated, they are each unique. Furthermore, Colquitt et al.'s investigation highlighted the differential aspects of interpersonal and informational justice, resulting in further support for the dissolution of the interactional justice construct. Therefore, the term "interactional justice" noted herein refers to both the 
interpersonal justice and informational justice constructs, rather than a distinct “interactional justice” construct.

Summary of Current Organizational Justice Theory

As a result of the consideration of the perceptions of outcomes, processes, interpersonal interactions, and the provision of information, organizational justice theory presents a relatively comprehensive view of individuals' reactions to organizational events. More importantly, organizational justice underscores the relevance of the decision processes used by organizational representatives, the final decisions rendered by organizational representatives, and the information and interpersonal treatment an individual receives during and after a decision process to an individual's motivation and future behavior.

\section{Extensions of Organizational Justice Theory}

\section{Fairness Theory}

Since the development of organizational justice theory as it is known today, the theory has been further extended to include more specific ways of measuring perceptions. For example, Folger and Cropanzano (2001) presented a theory of accountability known as "Fairness Theory." According to Folger and Cropanzano, three conditions must be met in order to elicit a fairness perception. First, an injurious condition must be present. Second, such an injurious condition must be attributable to an entity's discretionary behavior. Finally, such discretionary behavior must violate an individual's moral beliefs and/or ethical standards. 
In addition, three judgments are subsumed in relation to perceptions of fairness. Such judgments are referred to as "counterfactuals" and have been identified by Folger and Cropanzano (2001) as "would," "could," and "should" types of thinking. "Would" counterfactuals are concerned with imagined alternatives. The easier an alternative is to imagine, the more unfavorable the reaction to the event. For example, if an individual is denied bonus pay for his performance because the organization was not able to quantify his effort, but can easily imagine what would have happened had the company recognized his performance, he feels upset. On the other hand, "could" counterfactuals stress an individual's evaluation of the possible alternative actions that "could" have been taken by the entity that initiated the event. For example, if an individual is denied a pay raise, but believes that the organizations could have done a better job at assessing how much he deserved a pay raise, he feels slighted. Finally, "should" counterfactuals are ethical or moral in nature. "Should" counterfactuals are in regard to an individual's perception that an event "should" have occurred in a particular way or fashion. For example, if an individual is denied extra vacation days, but believes that the organizations should have given him extra vacation days to reward him for his ongoing commitment to organizational goals, he feels angry.

Related to the topics of interest in this dissertation, the counterfactuals described above were investigated in terms of applicant reactions by Gilliland et al. (2001). Their research indicated that different types of explanations using the counterfactuals presented in fairness theory had differential effects on justice perceptions. Specifically, "could" and "would" explanations affected justice perceptions and recommendation intentions. 
"Should" explanations, however, had to be combined with a "could" or "would" explanation in order for the explanation to have a positive effect on fairness perceptions and recommendation intentions. The results of this study are particularly important to the study of applicant reactions, as they demonstrate that different types of explanations have differential affects on fairness perceptions.

Further study of explanations by Shaw, Wild, and Colquitt (2003) explored explanation adequacy and provision using meta-analysis. Shaw et al. concluded that explanations had "powerful" effects on organizational justice perceptions. This study is further described in Chapter 4: Explanations Provided to Applicants.

\section{Fairness Heuristic Theory}

Another extension of organizational justice theory is known as Fairness Heuristic Theory (FHT) (Lind, Kray, \& Thompson, 2001). FHT rests on the assertion that "people use their judgments of fairness as a heuristic to guide decisions about the appropriate level of personal investment and involvement in groups, organizations, and institutions" (Lind et al., 2001). The theory assumes that people anticipate future interactions with organizational entities, and describes the process involved in making fairness judgments in relation to previously formed justice perceptions. In this way, the approach is sequential. According to FHT, there is a primacy effect such that early organizational events influence later perceptions of justice. The primacy effect can only be mitigated by a particularly salient action by the organization. As a result, the theory suggests that "policies or actions that seek to enhance feelings of fairness must ... be implemented early in the encounter" (Lind et al., 2001, p.191). 
Several studies have demonstrated support for the existence of a fairness heuristic. For example, van des Bos, Vermunt, and Wilke (1997) found that when fairness information timing was manipulated, information perceptions of a fair process predicted perceptions of fair outcomes and perceptions of fair outcomes predicted perceptions of fair processes. Their findings demonstrated that:

The order in which information about procedures and outcomes is received plays a crucial role in what people consider to be fair and that this can be found when people judge the fairness of a hypothetical procedure and an outcome as well as when they directly experience the fairness of a procedure and an outcome. (p.102) In this way, initial perceptions of fairness effect subsequent perceptions of fairness, providing an important segue for a newer conceptualization of organizational justice, known as the "event mediation approach." Newer Conceptualizations of Organizational Justice

Ambrose, Hess, and Ganesan (2007) introduced what they termed the "event mediation approach" to organizational justice. The event-mediation approach incorporates the sequential aspects of Fairness Hueristics Theory and challenges the current conceptualization of organizational as having four distinct dimensions, as identified in Colquitt et al.'s (2001) research. Ambrose et al.'s in-depth analysis of several common organizational justice models provided evidence to dispel the commonly held belief that the most parsimonious model under which organizational justice perceptions function involves orthogonally predictive dimensions of distributive, interpersonal, procedural, and informational justice. 
In 2001, Colquitt and colleagues proposed a four-factor model of organizational justice where (1) distributive justice and interpersonal justice affected event attitudes and (2) procedural justice and informational justice affected attitudes toward the organization. Ambrose et al. (2007) sought to determine the role of event-related attitudes in relation to organizational justice. The authors surveyed 285 people waiting for airplanes at an eastern international airport in the United States. The participants were asked to recall a customer-service related problem they had experienced over the past six months. Then, the participants completed various scales measuring justice perceptions, event attitudes, and system-related attitudes. Rather than supporting Colquitt et al.'s (2001) conceptualization of organizational justice, data analysis revealed that each organizational justice dimension predicted event-based attitudes and in turn, these attitudes mediated the relationship between the organizational justice dimensions and overall attitudes toward the organization.

The implications of this study are particularly pertinent to applicant reactions research, as it demonstrates the importance of organizational justice perceptions in relation to particular events rather than overall attitudes toward the organization. That is, the event (in this case, the selection process) becomes paramount to the prediction of future attitudes toward the organization, underscoring the importance of applicant reactions to such events/selection procedures. When studying Internet-based selection procedures, this approach is useful because it aligns the statistical testing of hypotheses with a sequence of events that are common in the real world. Specifically, this dissertation first measured privacy perceptions to determine how they affect applicant 
reactions to the Internet-based selection process. Then, fairness perceptions were measured. This structure allowed for manipulations of explanations and control to be studied in terms of their effects on both proximal outcomes (fairness perceptions) and distal outcomes (litigation intentions, organizational intentions, and test-taking motivation). Therefore, the true "power" of the manipulations was evaluated.

\section{Summary of Organizational Justice Approaches}

The multiple approaches to organizational justice provide for a way to better explain, understand, and predict behavior in organizations. Specifically, organizational justice theory helps explain individuals' reactions to organizational decisions. Fairness theory (Folger \& Cropanzano, 2001) provides a means of making sense of the conditions under which organizational justice perceptions are elicited. Fairness heuristic theory (Lind, Kray, \& Thompson, 2001) stresses the importance of primary events in formulating justice perceptions. The event-mediation approach (Ambrose, Hess, \& Ganesan, 2007) demonstrates the sequence of events in individuals' formulations of organizational perceptions. Taken together, these theories provide an overarching framework that is useful in studying applicant privacy concerns in relation to Internetbased selection procedures. 


\section{Chapter 3:}

The Study of Applicant Reactions

\section{Historical Approaches to Applicant Reactions Research}

Research on applicant reactions to employee selection procedures began as a small niche area of research in the late 1960s and early 1970s. Whereas several researchers in Industrial/Organizational Psychology, Organizational Behavior, and related fields had previously focused their efforts on appraising the utility and validity of employee selection procedures (Office of Strategic Service Assessment Staff, 1948; Smith, 1948), early applicant reactions research attempted to address the effects of using such methods. These authors began to examine the effects of "recruitment" practices on applicant behavior. Though present day conceptualizations of job applicant experiences often discern between the recruitment and selection processes, research prior to the late 1980 's viewed the selection process as a complimentary component to recruitment. For example, Rynes, Heneman, and Schwab (1980) wrote:

While the boundaries between recruitment and selection are admittedly somewhat fluid, the job seeker effectively remains a recruit until the point at which the organization decides to extend or withhold a job offer. Hence, the organization must use its recruiting process not only to attract applicants via its administrative procedures, but also to evaluate the probable satisfactoriness of each recruit as an eventual employee. This is commonly done through techniques such as the employment interview, pre-employment testing, and the analysis of information provided in various application materials required of the job seeker. (p. 531) 
Rynes et al (1980) thereby exposed the organizational perspective of applicant recruitment -- that organizations viewed recruitment as inclusive of the selection process due to the role an applicant plays before hire. However, the applicant may mentally distinguish between recruitment and selection. For example, an individual may dislike a recruiter, but may react favorably to a selection process; the two processes can be mutually exclusive. Therefore, two types of studies exist within the early applicant reactions literature: those that focus on applicant perceptions of the interviewer (Schmitt $\&$ Coyle, 1976) and those that focus on applicant reactions to the selection process (Downs, 1969; Matarrazo, Wiens, Jackson, \& Manaugh, 1970; Thronson \& Thomas, 1968). Given that modern organizational justice theory was under development and not yet suitable for application to this context, the omission of justice variables (e.g., interpersonal justice) that could possibly rectify this dissociation is not surprising. Within this dissertation, a discussion of how organizational justice encompasses these two types of studies will follow.

The majority of early applicant reactions research remained focused on applicant perceptions of interviewers (e.g., Schmitt \& Coyle, 1976) and applicant reactions to information they received about the job (job attributes; e.g., Taylor \& Bergman, 1987). However, there were several studies that focused on applicant perceptions of the face validity of selection procedures (e.g., Taylor \& Sniezek, 1984). The typical applicant outcome variable in these investigations was simple in that researchers measured individual "preference" for different selection procedures. For example, several studies highlighted that applicants had different preferences for the content of face-to-face 
interviews (Downs, 1969; Matarrazo, Wiens, Jackson, \& Manaugh, 1970; Thronson \& Thomas, 1968). Authors such as Taylor and Sniezek (1984) investigated the face validity of selection procedures when they assessed applicant attitudes regarding organizational attractiveness and opportunities to present job qualifications. The authors' findings suggested that when applicants were asked questions about general qualifications they felt that they had the opportunity to present their qualifications, which resulted in more positive applicant reactions than when such questions were not asked.

By the late 1980s, applicant reactions research began to question the effects that the selection process had on applicants at different time points during their experience. For example, Taylor and Bergmann (1987) identified several phases to the "recruitment process" for a real-world sample of college student job applicants. Such phases included (1) the [campus] interview, (2) the post [campus] interview phase, (3) the site visit, (4) the job offer, and (5) the job offer decision. Taylor and Bergmann proposed that there would be a significant relationship between recruitment activity and applicant reactions throughout the process. However, this relationship was significant in only the initial campus interview phase. Hence, Taylor and Bergmann (1987, p.281) concluded that “recruitment practices predicted applicants' reactions only at the initial campus interview stage. Recruiter demographic characteristics, interview characteristics, and applicants' perceptions of recruiter empathy were related to applicants' organizational attractiveness and the probability of their accepting the job offer." Still, the authors failed to recognize the relative importance of the selection tool itself, as they did not specifically measure perceptions of fairness of the process. They also did not measure perceived fairness of the 
outcome, as most applicant reactions researchers would today. Furthermore, the authors did not attempt to measure the incremental variance predicted by recruiter demographics, interview characteristics, and perceptions of recruiter empathy.

The final conclusions drawn by Taylor and Bergmann (1987) give insight into the early perspectives of applicant reactions research. The authors concluded that "in summary, we believe that recruitment activities influence applicants' reactions primarily through their impact on the individuals' inferences about job attributes. When applicants are able to obtain direct, even if limited, information about attributes (generally during later, more interactive recruitment stages), recruitment activities no longer appear to significantly influence their reactions" (p.282). In other words, Taylor and Bergman were attributing a lack of relationship between applicant reactions and later phases of the recruitment process to the receipt of information about job attributes rather than reactions to the actual process itself. Unknowingly, such researchers were only explaining one part of the applicant reactions puzzle. Whereas in some jobs learning about the job itself may be central to applicant reactions, such as in the case of police officer selection, in jobs where applicants have the opportunity to apply to multiple employers, the process may become central to applicant reactions.

As researchers began to explore applicant reactions to different types of selection procedures, the applicant reactions puzzle began to fit together. Much of the early applicant reactions research dealt exclusively with selection interviews (e.g., Taylor \& Sniezek, 1984), rather than other selection procedures such as written tests, work samples, or qualification and training reviews (e.g., Harris, 1989; Maurer \& Fay, 1988). 
This is probably because interviews were viewed as a typical method of employee selection. Still, much of the research in this area was concerned with interview validity rather than applicant reactions.

Validity of Employee Selection Procedures

The validity of a selection tool can be measured and thus defined in several different ways. Some of the most common calculations of validity for employee selection procedures include criterion-related validity, construct validity, and content validity (Gatewood, Feild, \& Barrick, 2007). As previously mentioned, early validity studies on employee selection tools involved assessing the ability of written tests to predict human performance on the job (Office of Strategic Service Assessment Staff, 1948; Smith, 1948). As researchers became interested in other selection tools, particularly the selection interview, they began exploring how such tools functioned to predict performance in a variety of jobs. The concept of validity is important because it provides a reason why practitioners use selection tools. A selection tool is considered "valid" if it is able to accurately measure the construct in question. For example, selection tools are designed to measure various individual attributes (i.e., personality, cognitive ability) in order to predict future performance on the job. Therefore, a common way to establish the validity of selection tools is to statistically compute the correlation between applicants' performance on the selection tool and job performance criterion, such as applicants' later ratings of performance on the job (criterion-related validity).

Still, another conceptualization of validity exists that sometimes escapes the typical research performed by selection procedure validation studies. Unlike criterion- 
related validity, construct validity, and content validity, where the typical goal of the said type of validity is success in making the best selection decision, as noted earlier, there exists the concept of face validity (Guion, 1965). Face validity, however, is the perception that a selection tool or process measures what it proposes to measure. Face validity refers to the perception that a test or process is related to the job to which an applicant applies (Gatewood, Feild, \& Barrick, 2007; Guion, 1965). While some earlier texts did discuss the idea of "face validity" and its importance to the use of selection tools, more sophisticated analyses of applicant perceptions such as that used in more recent "applicant reactions" research were unknown.

Previous research has demonstrated that particular characteristics of the selection process, such as the degree to which applicants see the procedure as valid and/or jobrelated, can affect reactions to the process (Kluger \& Rothstein, 1993; Ployhart \& Ryan, 1998; Smither, Reilly, Millsap, Pearlman, \& Stoffey, 1993). In fact, as early as 1946, test developers were concerned with the "face validity" of employee selection tools (Cleeton, Kraft, \& Royster, 1946). Face validity of selection procedures is particularly important to applicant reactions, as applicants who view selection tools as face valid are more likely to view the process favorably (Macan, Avedon, Paese, \& Smith, 1994; Steiner \& Gilliland, 1996). Interestingly, Steiner and Gilliland (1996) found a strong relationship between applicant beliefs that employers had a right to obtain information using a particular selection process and favorability. Given such results, the perceived face validity of a procedure should be taken into account when studying applicant reactions. This study 
measured perceptions of face validity as a component of the Selection Procedural Justice Scale (see Chapter 7: Method) to consider such perceptions.

\section{Modern Applicant Reactions Research}

A great deal of research has been completed regarding applicant reactions to selection procedures. Though some studies in the literature approach applicant reactions with Attribution Theory as the paramount theoretical orientation (e.g., Ployhart, Ryan, \& Bennett, 1999), most investigate applicant reactions through the lens of an organizational justice perspective (e.g., Arvey \& Sackett, 1993; Gilliland, 1993). Whereas attribution theory in applicant reactions research focuses on the placement of responsibility on entities involved in the selection process in order to alleviate cognitive dissonance (Ployhart et al., 1999), organizational justice most commonly refers to individuals' perceptions of the fairness of procedures (the hiring process) used to determine an outcome (e.g., Gilliland, 1993), individuals' perceptions of the fairness of the outcome itself (the hiring decision) (e.g., Gilliland, 1993), and individuals' perceptions of interpersonal treatment and the adequacy of the information provided (e.g., Gilliland et al., 2001).

\section{Applicant Reactions and Organizational Justice}

Applicant reactions research is concerned with the ability of organizations to elicit positive reactions from job applicants. A commonly accepted approach to measuring applicant reactions involves measuring organizational justice perceptions and specifically, applicants' perceptions of fairness. The Principles for the Validation and Use of Personnel Selection Procedures (Society for Industrial/Organizational 
Psychology, 2003), a guide commonly used by psychology practitioners in developing selection procedures, states that "Fairness has no single meaning, and, therefore, no single statistical or psychometric definition. Fairness, or lack of fairness, is not a property of the selection procedure, but rather a joint function of the procedure, the job, the population, and how the scores derived from it are used" (p.31). This definition demonstrates the importance of several variables affecting the fairness of selection procedures. Such fairness may lead to fairness perceptions of applicants and underscores the importance of multiple aspects of fairness. The Principles further asserts that: In addition to the organization's needs and objectives, researchers also need to consider the acceptability of the selection procedure to candidates. A number of factors influence candidates' reactions to a selection procedure, including individual characteristics (e.g., work experiences, demographics, and cultural backgrounds), the role of the individual (e.g., applicant, incumbent, manager), the extent to which the content of the selection procedure resembles the work, the individual's capability with respect to the constructs measured, and the perceived passing or selection rate. (p. 40)

Clearly outlined in the above statement are several factors that are considered important when evaluating selection procedures for their propensity to elicit positive reactions. Given the importance placed by the Principles for the Validation and Use of Personnel Selection Procedures on both fairness of selection procedures and applicant reactions to such procedures, an overall model that highlights the importance of several variables in applicant reactions is merited. 
Arvey and Sackett (1993) were among the first to put forth several hypotheses regarding an overall theoretical model of applicant reactions to selection procedures. In particular, Arvey and Sackett proposed that applicant perceptions of fairness were paramount to applicant reactions, going into detail about potential determinants of selection system fairness, including content, development, administration, context, and outcomes. Arvey and Sackett also pointed out the possible consequences of perceptions of unfairness among applicants, many of which were supported in later empirical research. Still, coverage of the topic area was limited and required further explication, which resulted in a well-known article by Gilliland (1993).

Gilliland (1993) proposed a comprehensive model of applicant reactions that incorporated organizational justice theory (Figure 1). In his article, Gilliland mapped Leventhal's (1980) ten procedural justice rules onto a proposed model of applicant reactions to the selection process. Gilliland identified three categories in which to group these rules which included formal characteristics of the selection process, explanations offered to applicants during the selection process, and interpersonal treatment perceived by the applicant.

As identified by Gilliland (1993), the formal characteristics grouping of procedural justice rules included the following rules:

Job-relatedness refers to the "extent to which a test appears to measure content relevant to the job situation or appears to be valid" (Gilliland, 1993, p.703). Research has demonstrated that particular types of tests elicit more positive perceptions of jobrelatedness than others. For example, Smither et al. (1993) found that cognitive ability 
tests were perceived to be more job-related than personality tests. Bauer, Maertz, Dolen, and Campion (1998) found that job-relatedness of tests were positively related to applicants' perceptions of fairness.

Opportunity to perform refers to a job applicants' ability to demonstrate their knowledge, skills, and abilities in a selection process (Gilliland, 1993). In applicant reactions research, opportunity to perform is believed to be related to the organizational justice concept of “voice.” (Arvey \& Sackett, 1993). Several studies have demonstrated that applicants' perceptions of the opportunity to perform are related to positive perceptions of the process (e.g., Bertolino \& Steiner, 2007; Nikolaou \& Judge, 2007; Schleicher et al., 2006). In the most comprehensive investigation of "opportunity to perform" to date, Schleicher, Venkataramani, Morgeson, and Campion (2006) found that opportunity-to-perform “does in fact drive fairness perceptions (confirming Gilliland's (1993) model and Truxillo et al.'s (2002) findings)" (p.580). Other research demonstrates strong, positive correlations between opportunity to perform and positive applicant reactions. For example, in a recent study by Bertolino and Steiner (2007), opportunity to perform was shown to be a strong predictor of fairness perceptions. Similarly, Nikolaou and Judge (2007) found a strong correlation between favorability of selection test type and opportunity to perform.

Reconsideration opportunity was described by Gilliland (1993) as an opportunity for a "second chance." Bauer et al. (2001) described the construct more explicitly when they defined it as "the opportunity to challenge or modify the decision making/evaluation process and the opportunity to review and/or discuss scores and scoring" (p.391). 
Consistency of administration refers to the standardization of the selection processes (Gilliland, 1993). Consistency of administration appears important to job applicants, as Ployhart and Ryan (1998) discovered that administration inconsistency was significantly related to negative fairness perceptions among applicants.

As identified by Gilliland (1993), the explanation variables grouping of procedural justice rules included the following rules.

Feedback often refers to both the timeliness and content of information given to applicants (Gilliland, 1993). Common approaches to determining the effect of feedback on applicant reactions involve the use of fairness theory (e.g., Folger \& Cropanzano, 2001). In such research authors have investigated the use of counterfactuals (Gilliland et al., 2001), specificity of explanations (e.g., LaHuis, Perreault, \& Ferguson, 2003), interpersonal sensitivity, (e.g., Mayer \& Ployhart, 2007), and timeliness (Waung \& Brice, 2000). Feedback is closely related to selection information. Participants in this study were provided feedback in the form of an electronic letter about the selection decision in this study. Selection information/explanations were provided based on a randomly assigned study condition (see Chapter 7: Method).

Selection information, according to Gilliland (1993), refers to the provision of justifications for decisions. The idea that selection information is related to organizational justice stems from the concept of informational justice as it was derived from Greenberg (1993). As previously noted, informational justice is proposed to capture individuals' perceptions of the explanations offered for outcomes. Whereas Gilliland (1993) considered selection information to be related to procedural justice, this study will 
consider it to relate to informational justice, consistent with current organizational justice research (i.e., Colquitt et al., 2001). Information regarding the use of the Internet-based selection procedure was used as a variable of interest in this study.

Honesty was proposed as a unique and distinct predictor of applicant reactions (Gilliland, 1993). Often, honesty and trustworthiness is viewed as a quality noticed in selection test administrators by applicants, as identified in research on employment interviews (Schmitt \& Coyle, 1976). Honesty is often an attribute applied to people/administrators of selection procedures. As a result of the absence of a person who served as a test administrator in this study, honesty was not explored.

As identified by Gilliland (1993), the interpersonal treatment variables grouping of procedural justice rules included the following.

Two-way communication involves an applicant's ability to share their views and opinions during the selection process (Tyler \& Bies, 1990). Two-way communication is similar to the feeling-sharing aspects of "voice" in that applicants experience more positive perceptions of the process when they are able to share how they feel about the process. Building on Thibaut and Walker's (1975) idea of process control, whereby individuals had the opportunity to voice their position to decision-makers, Tyler, Rasinski, and Spodick (1985) identified the "value-expressive" component of procedural justice. The value expressive component was said to involve an individual's ability to express his/her opinions and concerns without impact on the outcome of decisions. Tyler (1987) later refined this idea by demonstrating that value expression was most effective when individual's believed that their concerns were evaluated by decision makers. Based 
on this concept, "voice" emerged as a component of procedural justice, as evidenced in Gilliland's (1993) model.

The positive effects of voice have been demonstrated by several researchers (Lind, Kanfer, \& Earley, 1990; Lind \& Tyler, 1988; Tyler \& Lind, 1992). The voice effect has been explained by:

... presuming that persons given an opportunity to express their views will believe that voice will help them control their outcomes - their arguments might persuade the decision maker to provide a better outcome---and that these expectations lead to higher procedural fairness judgments. (Lind, Kanfer, \& Earley, 1990, p.952)

Voice has been shown to be related to positive perceptions of many human resource processes. For example, Cawley, Keeping, and Levy (1998) found that both valueexpressive participation and instrumental participation in performance appraisals were strongly correlated with overall reactions. Cawley and his colleagues asserted that their results were "consistent with a growing body of research in the justice literature suggesting that the opportunity to voice one's opinions regarding a decision increases the perceived fairness of the process, even if one does not influence the decision" (p. 625). Therefore, Cawley et. al. suggests that by simply allowing individuals to voice their opinions organizations may be able to increase positive perceptions of procedural justice. These same ideas can be applied to applicant reactions to selection processes. In fact, in early applicant reactions research, Bies and Shapiro (1988) allowed study participants in one condition to ask questions about the job and the organization during a simulated 
interview. Compared to applicants who did not have this opportunity, these participants had higher perceptions of procedural justice. Voice was not a key variable of interest in this study, but may be an area of possible future research.

Interpersonal effectiveness of the administrator is similar to honesty in that it deals with the perceptions of the individual(s) administering the selection procedure (Gilliland, 1993). The "warmth and respect" a test administrator demonstrates toward an applicant is believed to define the administrator's interpersonal effectiveness (Gilliland, 1993). Given the computer-based nature of the experiment manipulation, interpersonal effectiveness was not examined in this study.

Propriety of questions includes both "improper questioning and prejudicial statements" (Gilliland, 1993, p.709). Invasiveness of selection procedures may be related to propriety of questions in that certain types of questions or tests may be perceived as obtrusive by applicants. For example, Stone-Romero et al., (2003) found that certain types of selection procedures (i.e., drug tests) were viewed more negatively than other types of selection procedures (i.e., structured interviews) due to their perceived invasiveness. Invasiveness has been similarly studied by privacy researchers (e.g., Stone \& Stone, 1990), but did not serve as a key variable of interest in the present study. Rather, this dissertation will explore individuals' concerns regarding privacy of personal information and data that is provided during an Internet-based selection process.

According to Gilliland (1993), the above rules will mediate the relationship between characteristics of the selection process and overall perceptions of the selection process. Common characteristics of the selection process include test type, human 
resource policy, and human resource personnel. Gilliland proposed several relationships, such that formal characteristics were said to mediate the relationship between test-type and fairness perceptions, explanations were said to mediate the relationship between human resource policy and fairness perceptions, and interpersonal treatment was said to mediate the relationship between human resource personnel and fairness perceptions.

Gilliland (1993) described the outcome of the selection process to be "the most salient outcome" (p.715). Therefore, in addition to procedural justice rules, Gilliland also mapped commonly researched "distributive justice rules" that included the rules of equity, equality, and needs to his model. Gilliland's equity rule mirrored Adam's (1965) Equity Theory in that job applicants were said to evaluate their performance in terms of their inputs (effort) versus the gained outputs (the selection decision). Equality, however, focused more on equal opportunities for success than evaluations of inputs versus outputs. Finally, Gilliland identified needs as a distribution rule that captured the saliency of individuals' unique needs when evaluating outcomes, such as needs for accommodation in the case of disabled applicants.

The importance of perceptions of distributive justice should not be dismissed: It is necessary to point out that outcome favorability (i.e., selection versus rejection) is the key determinant of such perceptions (e.g., Ryan \& Ployhart, 2000). However, organizations are unable to control such perceptions without rendering a different hiring decision. Still, distributive justice perceptions are a key variable of interest in this study, as it was proposed to be a component of a mediating "Fairness Perceptions" variable. The present study did not manipulate the outcome of the selection procedure, as all applicants were 
rejected. It was expected that perceptions of fairness would be comprised of both perceptions of the procedure and the outcome/selection decision.

\section{Common Outcomes of Selection Fairness}

Of particular importance to applied contexts are the distal outcomes proposed by Gilliland in his model of applicant reactions. Perceptions of overall fairness of the selection process were proposed to mediate the relationship between procedural justice rules and reactions during hiring (job-application and job acceptance decisions, application recommendations, test motivation, legal battles), and reactions after hiring (performance, organizational citizenship behavior, job satisfaction, organizational climate). Perceptions of overall fairness of the selection outcome were proposed to mediate the relationship between the distributive justice rules and reactions during hiring, reactions after hiring, and self-perceptions (self-esteem, self-efficacy, future job-search intentions).

In order to test an overall model and summarize findings from the applicant reactions literature, Hausknecht, Day, and Thomas (2004) conducted a meta-analysis on applicant reactions studies. In their meta-analysis, Hausknecht et. al (2004, p.641) stated that "the basic premise of organizational justice theory in selection contexts is that applicants view selection procedures in terms these four facets of justice [distributive justice, procedural justice, interpersonal justice, and informational justice], and these perceptions influence future attitudes, intentions, self-perceptions, and behaviors." The Hausknecht et. al model was important because it demonstrated the unique contribution of each type of justice (i.e., distributive justice, procedural justice, interpersonal justice, 
information justice) to commonly studied applicant reactions variables. Overall, Hausknecht et. al. supported Gilliland's (1993) model of application reactions and confirmed that organizational justice was a relevant, appropriate theory on which to base applicant reactions research. Specifically, formal selection process characteristics (such as job relatedness and opportunity to perform), explanation variables (such as selection information), and interpersonal treatment in general were found to be most important in terms of applicant reactions.

Research has identified several benefits of eliciting positive applicant justice perceptions. Much of this work originated from Gilliland's (1993) article in which he proposed that perceived justice has an impact on applicant reactions during hiring, applicant reactions after hiring, and self-perceptions (see Figure 1). More recent research discovered that positive perceptions of fairness are related to support for the organization (Brockner, Paruchuri, Idson, \& Higgins, 2002). Positive perceptions of selection procedures among job applicants has been shown to be related to perceptions of the organization, recommendation intentions, and job acceptance intentions (Hausknecht et al., 2004), further underscoring the importance of such perceptions.

Of the many possible outcomes of applicant reactions, this study focused on five. The five applicant reactions outcomes explored in this study were carefully selected based on their potential impact to organizations. Such outcomes have been explored in previous research, including meta-analytic investigations by Hausknecht et al. (2004). These outcomes included litigation intentions, recommendation intentions, job acceptance intentions, purchasing intentions, and test-taking motivation. 


\section{Litigation Intentions}

Over the years, selection processes perceived as unfair by applicants have resulted in widely publicized court proceedings, including a recent case where gender-bias was alleged by a group of over one-million female employees (Dukes v. Wal-Mart Stores, Inc., 2010). Thus, a major concern for many organizations is the possibility of litigation stemming from the use of selection procedures. Outside of applicant reactions research, the relationship between organizational justice perceptions and legal claiming has been highlighted. For example, Goldman (2003) demonstrated that interactions between justice perceptions predict legal-claiming following an employee's termination. Goldman, Paddock, and Cropanzano (2004) examined the content of employee complaints about organizations (focused on multiple targets) and provided further support to the finding that organizational justice perceptions were related to incidents of legal claiming behavior. Still, in applicant reactions research, few authors have been able to study actual legal claiming behavior as a variable, as there are multiple barriers to measuring this phenomenon in applied research. Such barriers include perceptions that organizations participating in such research studies will inadvertently encourage legal claiming by placing the idea into the minds of applicants who would not have pursued litigation otherwise. Instead, applicant reactions researchers have focused on applicants' intentions to litigate, and usually in laboratory settings. For example, Bauer et al. (2001) used a study design whereby participants imagined themselves applying for a job. The participants then responded to questions regarding their likely intentions to litigate following the outcome of the selection process. Results from the Bauer et al. study 
demonstrated that intentions to litigate were positively correlated with negative fairness perceptions regarding the selection process. Given the difficulties in assessing actual litigation behavior as well litigation intentions in real-world contexts, the proposed study employed a more psychologically salient experimental context in order to measure applicant litigation intentions among a sample of study participants.

\section{Recommendation Intentions}

Selection practices may have broader consequences than simply the effects on the applicant. Consistent with this notion, recommendation intentions are considered to be a possible "spillover effect" of applicant reactions, as they have been described as an impression that is "transmitted from current to potential applicants, thus affecting the organization's general image or reputation" (Rynes \& Barber, 1990, p.290). Researchers have demonstrated that these effects are real, and have shown that applicant reactions may affect whether not an applicant will recommend the organization to other potential job applicants (e.g., Gilliland, 1994; Hausknecht et al., 2004; Ployhart \& Ryan, 1998; Smither et al., 1993). In their meta-analysis, Hausknecht and colleagues found a strong relationship between applicant reactions (i.e., procedural justice perceptions, distributive justice perceptions, attitudes toward tests) and recommendation intentions $(K=17, r=$ .52). Studies in their meta-analysis included those performed by Ployhart and Ryan (1998), who found a positive relationship between perceptions of fairness and recommendation intentions when applicants perceived a fair process.

Among the authors in the Hausknecht et al. (2004) meta-analysis who found similar effects, Smither et al. (1993) found that perceived predictive validity was 
positively related to recommendation intentions one month after applicants had participated in the selection procedure. Unlike Gilliland (1994), Smither and colleagues failed to design a study that sought to mitigate the effects of applicant reactions on recommendation intentions. Fortunately, Gilliland (1994) went a step further than Smither et al. (1993) when he found a positive correlation between perceptions of fairness and recommendation intentions. Though the correlations he found were fairly weak (i.e., process fairness, $r=.30$; outcome fairness, $r=.23$ ), Gilliland found that those who were rejected via the selection process but who also received an explanation indicated that they had significantly higher recommendation intentions than those who were rejected and did not receive an explanation. Similarly, this dissertation tested the moderating effects of explanations, as manipulated in both pre-test information and notifications of the hiring decision, on applicant reactions and resulting recommendation intentions.

\section{Job-Acceptance Intentions/Organizational Attraction}

One of the most important outcomes of applicant reactions is whether or not an applicant accepts a job offer upon its receipt. This is intuitive, as employers would not want to "turn off" accepted applicants to their respective organizations. Research on job acceptance intentions and applicant reactions have shown mixed results, in part because of the mixed use of applicant reactions variables. The Hausknecht et al. (2004) metaanlaysis found a moderate correlation between several applicant reactions variables and job acceptance intentions $(K=13, r=.33)$. Among the studies included in their metaanalysis, Smither et. al (1993) found that there was a strong relationship between 
applicant reactions (perceptions of face validity) and applicant attraction to the organization, which included aspects of attraction such as job-acceptance intentions. Unlike Smither et al., Ployhart and Ryan (1998) found that job acceptance intentions were unaffected by applicant reactions (perceived fairness of the process). Ployhart and Ryan asserted that "intentions to accept the job were high regardless of how the selection procedures were administered" (p.14). On the other hand, a prior study by Macan et al. (1994) demonstrated that applicant reactions variables were related to job acceptance intentions. However, there was one caveat to their findings- applicants' impression of the organization as a whole, not just the selection process, served as a "key predictor" of how job acceptance intentions. Macan et al. investigated further and found an interaction effect. Applicants who participated in the selection process and who held more favorable impressions of organization "indicated more favorable job acceptance intentions than those with less favorable intentions" (p.732-733).

Given the mixed results demonstrated in the above studies, it was important to carefully hypothesize specific relationships between applicant reactions variables and job acceptance intentions. Therefore, this dissertation sought to discern which applicant reactions variables were most relevant to job acceptance intentions. Specifically, I examined relationships between fairness perceptions and a higher order factor comprised of job acceptance intentions. I hypothesized that as perceptions of fairness increased, job acceptance intentions would also increase. 
Reapplication Intentions \& Reapplication Behavior

Though not directly measured in this dissertation, applicants' plans to reapply for future jobs with an organization are known as their reapplication intentions. Gilliland et al. (2001) went a step further and studied actual reapplication behavior with a real-world sample of 32 tenure-track professorship applicants who had the opportunity to reapply for a similar position within the same organization the following year. Reapplication behavior was described by Gilliland et al. (2001) as a behavior that may be beneficial to many organizations. The authors pointed out that:

Although reapplication behavior may not always be a good thing, particularly if the rejected candidates lack necessary qualifications, in times of low unemployment, reapplication from qualified candidates who failed to receive a job because of a hiring freeze represents a valuable addition to the applicant pool. In addition, some applicants who lack the qualifications for the immediate position may be highly qualified for future positions. Organizations do not want to discourage reapplication from these individuals. (p. 677)

Candidates who apply for one job may fail to be the most qualified. However, this does not mean that they are not jobs for which they would be the best candidate. Therefore, reapplication behavior becomes important to organizations; specifically, organizations with high volume hiring. For example, if an individual applies for a position as a sales associate in a sporting goods department of a major retail chain, he/she may be rejected due to his/her lack of knowledge of sporting goods equipment. However, the individual may be well qualified and the best candidate for a position in the apparel department. 
Research has demonstrated that not only do applicant reactions affect individuals' reapplication behavior (Gilliland et al., 2001), but they also affect individuals' reapplication intentions (Ployart \& Ryan, 1998). Ployhart and Ryan found a weak, positive correlation $(r=.14)$ between applicant fairness perceptions and reapplication intentions. In studying behavior, Gilliland and colleagues discovered that justice perceptions could affect reapplication behaviors, as they found that 24 of 32 original applicants reapplied for a similar position with the same organization the next year. Given these findings, it is sensible to assert that applicant reactions can affect reapplication intentions and may cut-off a potential supply of valuable, qualified candidates. This dissertation did not measure reapplication behavior or intentions, specifically, but did seek to measure other organizational intentions variables that are believed to be highly related.

\section{Test-Taking Motivation}

Test-taking motivation has also been identified as an outcome of applicant reactions to selection procedures. For example, Arvey, Strickland, Drauden, \& Martin (1990) examined test-taking motivation in relation to test-taking attitudes. Their research is notable because they were the first to measure these two constructs directly through the use of a sample of actual test-takers. The studies within the Arvey et al. (1990) publication created and validated a measure of test-taking attitudes that included testtaking motivation, known as the Test Attitude Survey (TAS). Overall, results from the investigation indicated that the motivation of test-takers affected test performance and test performance affected the motivation of test-takers. 
After several studies yielded results that supported the validity of Arvey et. al's TAS instrument (e.g., Chan, Schmitt, DeShon, Clause, \& Delbridge, 1997; Schmit \& Ryan, 1992; Schmit \& Ryan, 1997), Sanchez, Truxillo, and Bauer (2000) sought to develop a more robust, multidimensional assessment of test-taking motivation in order to "ask more specific questions about why and in what ways some people are more motivated to take a test than others" (p. 740). As a result, Sanchez and his colleagues (2000) used the Valence-Instrumentality-Expectancy (VIE) theory of motivation (Vroom, 1964) as a basis for their Valency, Instrumentality, Expectancy Motivation Scale (VIEMS). The VIEMS was used as a scale within this study to measure test-taking motivation as an outcome in the overall model of applicant reactions.

Support for the Organization through Purchasing Intentions

Similar to recommendation intentions, Rynes and Barber (1990) indicated that support for the organization is another important spillover effect of applicant reactions. Support for the organization may manifest itself in the form of consumer behavior or general dialogue with others regarding the organization.

Few studies have examined the "support for the organization" spillover effect. However, a study by Macan and colleagues (1994) called their results "consistent with consumer behavior research" as their results showed that applicant reactions are related to whether or not an applicant chooses to patron an organization (p.733). Macan et al. suggested that their findings were in-line with previous research because of the link between intentions and behavior, such that market research has "consistently found that to predict and understand purchasing intentions, intentions toward the specific acts of 
buying or using a product, rather than a general attitude toward the product, need to be assessed" (p.733). Hence, a link between consumer intentions and applicant reactions is implied, and was studied further in this dissertation.

Internet-based Selection Procedures

Much of the modern literature concerning applicant reactions investigates the issue as it relates to commonly used types of procedures such as behavioral interviews and paper-and-pencil based tests (e.g., Gilliland et al., 2001; Ployhart \& Ryan, 1998; Truxillo, Bauer, Campion, \& Paronto, 2002). Technology has altered the applicant experience, as more applicants apply for jobs using Internet-based selection systems. As a result, researchers have identified a greater need for applicant reactions research related to such systems (Bauer et al., 2006; Harris et al., 2003; Sinar \& Reynolds, 2001). Harris et al. (2003), Sinar and Reynolds (2001), and more recently, Bauer, Truxillo, Tucker, Weathers, Bertolino, Erdogan, and Campion (2006), were among the first to investigate applicant reactions to Internet-based selection procedures.

Harris et al. (2003) investigated applicant reactions to Internet-based selection procedures but focused on cross-cultural differences in privacy perceptions. Specifically, Harris and his colleagues proposed that privacy perceptions, Internet knowledge, and country of participant origin would be related to reluctance to submit personal information over the Internet. Their investigation demonstrated that applicants' knowledge level of the Internet was related to privacy concerns in that individuals who rated themselves as knowing a lot about the Internet were less likely to be concerned that their personal information would be handled inappropriately. Their results are important, 
as they suggest that familiarity with the Internet results in fewer privacy concerns when applying to jobs using the Internet.

Sinar and Reynolds (2001) focused their efforts on identifying the key themes of reactions to Internet-based selection procedures. Additionally, these authors connected reactions and outcomes such as satisfaction. Though their study was a minor addition to the literature, it provided for a deeper understanding of the specific types of reactions elicited from Internet-based selection procedures.

More recently, Bauer et al. (2006) examined applicant reactions to Internet-based screening procedures in both a laboratory study and a field study. Their results indicated that privacy perceptions predicted perceptions of fairness. Specifically, structural equation modeling yielded results that supported an overall model where procedural justice partially mediated the relationship between privacy concerns and outcome variables that included test-taking motivation, organizational attraction and intentions toward the organization. Still, the Bauer et al. (2006) study examined screening procedures rather than selection procedures. Therefore, individuals who passed the screening did not necessarily get hired for the job, which could affect overall procedural justice perceptions.

The three aforementioned studies established a foundation on which to base further investigation of applicant privacy reactions to Internet-based selection procedures. These studies demonstrated that Internet-based selection procedures provide a unique context in which to study applicant reactions (Sinar \& Reynolds, 2001), that privacy perceptions may have an effect on applicant behavior such as willingness to complete 
Internet-based applications (Harris et al., 2003), and that privacy concerns affect justice perceptions and other important organizational outcomes (Bauer et al., 2006). 


\section{Chapter 4:}

\section{Explanations Provided to Applicants}

Research on the provision of explanations has focused on increasing positive applicant reactions while mitigating the negative effects of decisions and processes. Given such focus, it is not surprising that this research stream fits well with organizational justice theory. Pertinent to this study, explanations can be used as a way to aid organizations in reducing the negative effects of rejecting applicants; that is, explanations have been explored as a means to mitigate negative effects of selection decisions (Truxillo, Bodner, Bertolino, Bauer, \& Yonce, 2009). Types of explanations, explanation provision and adequacy, and the power of explanations will be explored here in order to demonstrate how explanations are an important variable in applicant reactions research.

\section{Types of Explanations}

Explanations may be said to fall into one of two categories. As identified by Scott and Lyman (1968), explanations are classified as either justifications or excuses. According to Scott and Lyman:

Justifications are accounts in which one accepts responsibility for the act in question, but denies the pejorative quality associated with it ... Excuses are accounts in which one admits that the act in question is bad, wrong, or inappropriate but denies full responsibility. (p. 47)

Scott and Lyman further describe justifications as "socially approved vocabularies that neutralize an act or its consequences when one or both are called into question... 
Justifications recognize a general sense in which the act in question is impermissible, but claim that the particular occasion permits or requires the very act” (p.51). For example, in the context of applicant reactions, an organization may notify an applicant that he/she was not selected because the applicant did not obtain as high a score as other applicants on a valid assessment. Therefore, the organization justifies a decision that would be recognized by an applicant as negative by presenting a situation that would require such a decision in the minds of most applicants. Alternatively, excuses are "socially approved vocabularies for mitigating or relieving responsibility when conduct is questioned" (Scott \& Lyman, 1968, p.47). Thus, an organization may issue a notification of rejection to an applicant with an explanation that notifies that applicant that the organization could only afford to hire one applicant, even though two or more applicants were viable candidates.

The research of Scott and Lyman (1968) is important because it influenced applicant reactions researchers who searched for a way to increase positive applicant reactions. For example, Gilliland et al. (2001) cited the work of Scott and Lyman when developing a framework for providing explanations to applicants. The synthesis of the “procedural justice rules" and Scott and Lyman's proposed use of justifications and excuses provided a means to formulate theory-driven explanations to applicants. Researchers like Shaw and his colleagues (2003) have found positive relationships between explanations and justice perceptions; justifications and excuses have different strengths of effects on justice perceptions, such that excuses are more powerful. 


\section{Explanation Provision and Adequacy}

Research on explanations has lacked clarity due to mixed research findings and a lack of consistency in the application of the construct as a study variable. Still, Shaw et al. (2003) were able to examine research on explanations and identify commonalities in order to conduct a meta-analytic investigation. Shaw and his colleagues' work recognized that the most common way to measure the explanation construct is by assessing whether or not an explanation is provided (e.g., explanation provision) and/or by assessing whether or not an explanation is adequate (e.g., adequacy). Explanation provision is defined as "the extent to which an explanation is given for a decision" (Shaw et al., 2003, p.444). For example, in a study that assesses the effects of explanations on applicant reactions, explanation provision would usually be manipulated by either providing an explanation of selection/rejection to an applicant or withholding such an explanation. Explanation adequacy is defined as "the extent to which provided explanations are clear, reasonable, and detailed" (Shaw et al., 2003, p.444). Explanation adequacy is related to content of explanations rather than the actual provision of such information. In the previous example, explanation adequacy would usually be manipulated by varying the content of explanations provided to applicants regarding their selection or rejection. Specifically, whereas some individuals may receive a brief explanation that states what the test was purported to measure, other individuals may receive more comprehensive explanations that speak to the validity of the selection procedure, rendering the explanation as more "adequate." 
Explanation adequacy may be even more involved than it appears. For example, explanation adequacy may include perceptions of the style with which information is communicated. Providing explanations to individuals requires communication, and such communication is judged based on its perceived fairness. In fact, Bies and Moag (1986) proposed that people judged the fairness of communication on four principles that included truthfulness, respect, propriety of questions, and justification. They stated that "for most people, fairness requires that decisions be justified so that the action might be understood and found acceptable" (p.50). Truthfulness and respect appear to be more stylistic, whereas propriety of questions and justifications appear to have more substance. Explanations research that is focused on the style of information sharing is scant. However, several researchers have explored the use of explanations involving sensitive notifications (e.g., Ployhart et al., 1999; Yonce, 2005). Explanations research that is focused on the substance of explanations has been more popular in the research than research on style (e.g., Gilliland et al., 2001; Horvath, Ryan, \& Stierwalt, 2000). Such research received more attention when Shaw and his colleagues (2003) conducted a comprehensive meta-analysis on explanations.

\section{The Power of Explanations}

Shaw et al. (2003) summarized the use of explanations in a meta-analytic investigation of explanations across a range of contexts (i.e., not primarily in the context of applicant reactions). Shaw et al.'s investigation identified the effects of explanations on individual perceptions of organizational justice. Shaw and his colleagues analyzed 54 independent samples that included the work of Gilliland (1994), Gilliland et al. (2001), 
Ployhart et al. (1999), although the preponderance of the studies used were not in the applicant reactions context. Shaw et al. then coded the manipulations for several key variables. Dependent variables included distributive justice and procedural justice, independent variables included explanation provision and explanation adequacy, and moderating variables included type of explanation, outcome favorability, and context. Results demonstrated that providing explanations was significantly related to procedural justice $(r=.28)$ and distributive justice $(r=.21)$. The perceived adequacy of explanations demonstrated a strong relationship with justice variables, as it was significantly related to procedural justice $(r=.49)$ and distributive justice $(r=.40)$. Moderating variable analyses indicated that the effects of explanations were stronger with excuses versus justifications $(r=.39)$.

From Shaw et al.'s (2003) results we can assume that both the provision of explanations and increased explanation adequacy are related positively to justice perceptions. Furthermore, excuses rather than justifications appear to be more powerful types of explanations. Shaw et al.'s meta-analysis was limited as it did not fully capture the nuances of how explanations function in the context of applicant reactions. In fact, Truxillo and his colleagues (2009) asserted that the Shaw et al. meta-analysis "does not adequately summarize the specific literature on giving explanations to job applicants" (p.5).

\section{The Effect of Explanations on Applicant Reactions}

As indicated, several researchers have explored the use of explanations in the context of selection procedures. Such researchers have used a variety of different 
manipulations of the explanations variable and have obtained varying results. Some of the most cited works in the area of explanations research and applicant reactions have included studies by Gilliland (1994), Ployhart et al. (1999), Gilliland et al. (2001), and Truxillo et al. (2002).

Gilliland (1994) was among the first to study the use of explanations to mitigate negative applicant reactions. Gilliland used a high-fidelity laboratory study where undergraduate students completed a selection procedure for a paid, project-based research assistant position. Gilliland manipulated explanations such that a justification focused on job-relatedness was provided to applicants as an explanation for the use of the selection test versus no explanation. Results indicated that only recommendation intentions of those who received an explanation were significantly higher than those who received no explanation. Those who received an explanation perceived no significant difference in fairness perceptions compared to those who received no explanation.

Ployhart et al. (1999) designed a slightly more complex study that tested more than one type of explanation. The authors presented scenarios to student participants who were asked to imagine that they were applicants for a job, and then participants were asked to respond to letters that rendered a hiring decision and varied in terms of information-sharing (explanations). Manipulations included a "procedural information" condition, in which the selection procedure is described, a "justification information" condition, in which the organization justifies a selection decision in order to avoid blame, a "personal information" condition, in which a reason related to the applicant's own performance is provided, and a no explanation condition. Results indicated that providing 
rejected applicants with procedural information explanations and personal information explanations were related to more positive perceptions of fairness than when applicants were provided with no explanation. There were no significant differences in fairness perceptions between individuals who received justification information explanations and no explanation.

Gilliland et al. (2001) went beyond the work of Ployhart et al. (1999) by using two scenario-based studies and one field study to explore the effects of rejection letters (containing explanations) that were constructed using counterfactuals outlined by fairness theory (see Chapter 2). Consistent with fairness theory, "would," "could," and "should" explanations were crafted for rejected applicants. Results indicated that explanations designed to explain the superior qualifications of other candidates (would explanations) and explanations that focused on a lack of ability to hire a candidate (could explanations or excuses) were better than explanations focused on the appropriateness of the selection process (should explanations or justifications). Should explanations were not significantly different than no explanations in predicting fairness perceptions.

Truxillo et al. (2002) conducted a longitudinal field study in which information was varied within explanations provided to applicants in a real-world selection process. Given the real-world observation that human resource staff observed, Truxillo and colleagues designed a manipulation that focused specifically on individuals' reactions to the video-based test that applicants' completed. Job-relatedness and feedback timeliness were the focus of the explanation-information manipulation. Results indicated that the 
explanation condition provided in the study resulted in more positive fairness perceptions than the no explanation condition.

In response to increased research on applicant reactions and lingering questions regarding the effects of explanations on specific applicant reactions outcomes, Truxillo and his colleagues (2009) conducted a meta-analysis on 25 independent samples. Consistent with the findings of Shaw et al. (2003), explanations were found to have a significant effect on fairness perceptions $\left(Z=5.31, M_{r}=.12, p<.01\right)$. Pertinent to the present study, Truxillo and his colleagues also found that explanations had an effect on applicant perceptions of the organization $\left(Z=2.10, M_{r}=.06, p=.04\right)$ and test-taking motivation $\left(Z=5.80, M_{r}=.21, p<.01\right)$.

Given the results of Truxillo et al.'s (2009) meta-analysis, the present study investigated the effect of explanations on applicant reactions including fairness perceptions, perceptions of the organization, and test-taking motivation. Specifically, explanations were hypothesized to moderate the relationship between privacy concerns and fairness perceptions, which were expected to affect distal outcomes including litigations intentions, organizational intentions, and test-taking motivation. In the context of Internet-based selection procedures, explanations include information that addresses the privacy concerns of applicants (i.e., "privacy justifications") as well as provide reasoning for test usage (i.e., “excuse” explanations), consistent with Gilliland (2001).

This dissertation explored the provision of explanations to applicants participating in an Internet-based selection process. From previous research it is known that two variables affect the effectiveness of explanations- provision and adequacy. At the most 
basic level, the provision of explanations is more advantageous to organizations than a lack of provision (Shaw et al., 2003). A more complex issue is the adequacy of explanations, which is directly related to explanation content. As demonstrated by previous authors, explanation content can be varied in many ways. The most popular way to date has been to provide justifications or excuses to applicants. Though Shaw found that excuse explanations were more powerful than justification explanations over a number of HR contexts, I proposed that either type of explanation would be helpful to the applicant reactions context, especially if justifications take into account the specific needs and privacy concerns of applications. That is, privacy justification explanations, when taking into account the specific concerns of applicants, will be related to more positive fairness perceptions. Consistent with Truxillo et al. (2002), addressing specific concerns of applicants appears to be powerful in the mitigation of negative applicant fairness perceptions when using explanations. Given the unique context of Internet-based selection, explanations specifically designed to address privacy concerns should be similarly powerful to excuse explanations in mitigating negative applicant fairness perceptions. 


\section{Chapter 5:}

\section{Privacy Perspectives}

Organizations often request, and sometimes require, applicants to share personal information as a part of the application process. Such personal information may include current and previous addresses, social security numbers, and wage and income information. All of this information may be requested by employers and submitted by applicants via the Internet. Applicants' perceptions of privacy often stem from their reactions to such requests (Alge, 2001). Furthermore, the selection tools (e.g., personality-based tests, work history forms, interview questions) used to assess applicants for jobs may be perceived as invasive (Stone-Romero et al., 2003). Research has demonstrated that several variables influence perceptions of privacy including control (Eddy, Stone, \& Stone-Romero, 1999), level of invasiveness of selection procedures (Rosenbaum, 1973; Stone-Romero et al., 2003), and the possible misuse of personal information shared in the job application (Harris et al., 2003). This dissertation will explore how perceptions of privacy affect applicant reactions including both fairness perceptions and more distal outcomes (e.g., litigation intentions, recommendation intentions). As demonstrated in this review, negative applicant reactions are important to consider because they may be related to withdrawal of candidates from the selection process, a lack of positive recommendation intentions, and lower commitment among those who accept the job (Connerly et al., 2001). 


\section{Organizational Privacy}

In the field of Industrial/Organizational psychology, Stone and Stone (1990) were among the first to posit a comprehensive definition of "organizational privacy." They proposed that organizational privacy is:

... a state or condition in which the individual has the capacity to (a) control the release and possible subsequent dissemination of information about him or herself, (b) regulate both the amount and nature of social interaction, (c) exclude or isolate him or herself from unwanted (auditory, visual, etc.) stimuli in an environment, and, as a consequence, can (d) behave autonomously . . . in a context of an individual's dealings with organizations. (p.358)

Under this definition it is apparent that control over information and interaction is an integral component to perceptions of privacy. For example, individuals' privacy perceptions about personnel selection procedures may be altered by their ability to control the information released to the organization. Often, information required by the organization may be different from the information an individual wishes to provide the organization. Stone and Stone (1990) highlighted this disjunction when they identified competing interests between organizations and individuals within organizations. Stone and Stone asserted that the ways in which organizations use information about individuals may be perceived as a threat to an individual's perceptions of privacy. Individuals who participate in selection procedures may be aware of such competing interests and may experience different types of privacy concerns based upon the type of 
information being collected, the reasoning for the information collection, and the method through which the information is collected (Stone \& Stone, 1990).

Stone and Stone's (1990) proposed model has been supported by research studies conducted both prior to and following the publication of their review. Over twenty years ago, Stone, Guetal, Gardner, and McClure (1983) examined beliefs, values, and attitudes about information privacy in organizations. From their results, Stone et al. (1983) suggested that individuals view control over their personal information as important. Eddy, Stone, and Stone-Romero (1999) confirmed this assertion when they found that individuals who had control over who could view or handle their private information felt as though their privacy was less invaded and that the procedures used in reviewing their information were fair.

However, with the advent of the information age, individuals may view the electronic submittal of information with skepticism and may believe that they have significantly less control over how and by whom their personal information is used. The very nature of submitting an application with personal information over the Internet may have an effect on the applicant's privacy perceptions. Additionally, the fact that the Internet may be viewed as being more susceptible to unanticipated disclosure of information to external entities (individuals or organizations for whom the information is not intended) may have an effect on a job applicant's fairness perceptions. This would be consistent with Eddy et al.'s (1999) finding that individuals who believe that their information will be released to external entities will see the disclosure of their personal information as more invasive and will view the procedures used in reviewing their 
information as less fair. Given the findings regarding the control and release of personal information, this dissertation sought to determine whether or not perceived control over the dissemination of personal information when applying for jobs using the Internet had an effect on individuals' overall fairness perceptions of the selection process.

\section{Electronic Monitoring}

Within the broader study of organizational privacy, the specific ways in which privacy perceptions emerge is of interest to researchers. For example, individuals have long been skeptical of the ways in which organizations obtain information about current and prospective employees. Though beyond the scope of the present investigation, employee monitoring via Electronic Performance Monitoring and Control Systems (EPMCSs) and other Information Technologies are examples of information collection methods that have received a significant amount of attention from researchers (e.g., Alder, Noel, \& Ambrose, 2006). This is due to criticisms based on the perceived infringement of employee rights (Alder et al., 2006). Specifically, critics contend that electronic monitoring is in conflict with privacy rights (Alder et al., 2006). Although not directly related to privacy among applicants, the investigation of employee reactions to such monitoring provides insights into how applicants might perceive privacy issues. Though little academic research is available in this area, authors such as Alder and colleagues (2006) and Ambrose and Alder (2000) examined how monitoring affects employee attitudes toward the organization. Other research has demonstrated that more common methods of employee monitoring, such as supervisor observation, is perceived as less invasive than electronic means of monitoring employees (McNall \& Roch, 2007). 
Such research demonstrates the skepticism that employees (or prospective employees) may feel when using organizations' computer systems, which could translate into utilizing Internet-based selection systems to apply for jobs. Therefore, simply utilizing a computer-based resource to apply for a job with an organization may predispose a job applicant to having more negative perceptions of the selection process, at least in terms of privacy issues. This could be particularly true among those applicants who have greater concerns with privacy to begin with. This dissertation measured whether or not applicants preferred Internet-based selection processes or pencil-and-paper-based selection processes through questions asked following the experiment. This information was examined as a possible control variable.

Privacy in Personnel Selection

Research on privacy in personnel selection is not a new concept; researchers examined this issue as early as 1973 (e.g., Rosenbaum, 1973). Early research on privacy in the selection process focused on test content. For example, Rosenbaum (1973) investigated applicant reactions to different types of questions used in job interviews. Rosenbaum sought to determine whether or not particular types of questions were considered invasive to applicants' perceptions of personal privacy. Five groupings of questions were found that included (1) questions about family background and influences, (2) questions about personal history, (3) questions about personal interests and values, (4) financial management questions, and (5) questions about social adjustment. Results indicated that inquiries into applicants' management of finances and family background were seen as most invasive to privacy. Still, conclusions from this 
study are difficult to generalize to the present employment environment because (1) several questions in each of Rosenbaum's groupings are now illegal to ask of job applicants (i.e., questions about ethnic origin in the "Questions about Family Background" grouping) as outlined in Title VII of the Civil Rights Act of 1964 and (2) this study was completed before Internet-based applications were utilized. Simply stated, current policy and new technology present new questions regarding the privacy concerns of applicants.

As suggested, the advent of personnel selection systems that utilize Internet-based technologies to transmit data has added new complexity to the issue of applicant privacy by introducing a testing medium that was previously unfamiliar. Moreover, the transmission of data across geographical boundaries is more commonplace because it is more easily facilitated when it is done by electronic means. As Internet-based selection procedures become more common, answering questions regarding job applicants’ perceptions of privacy becomes more important than ever before.

Currently, the issue of personal data security has become a topic of scrutiny by mass media sources and has been highlighted in news reports that describe issues faced by both current and potential employees. As organizations and applicants began to increasingly use the Internet for job application purposes, individuals began to raise concerns regarding the use of the Internet for this purpose. In 2001, the Washington Post published an article titled "Report Criticizes Monster.com; Privacy Advocates Say JobSearch Site Misuses Data" (Johnson, 2001). This article covered a report issued by the Privacy Foundation, a nonprofit privacy advocacy group, which asserted that applicants 
who place their work histories and other personal information on the Internet using Monster.com inadvertently make their information available to other entities. The article further stated that "people who search for work via the Internet have long complained that they expose themselves to unwanted telephone calls and sales pitches -- not to mention threats from current employers that find out they are job-hunting" (p. 4E). More recently, news reports about the theft or disappearance of electronic employee records surfaced. In 2007, USA Today reported on the "disappearance of a computer drive holding personal and banking records of 100,000 Transportation Security Administration employees" (Frank, 2007, p.13A). Data on the missing hard drive was said to include names, addresses, and social security numbers. In 2009, Monster.com found itself in the news again when "Thieves took user IDs, passwords, e-mail addresses, names, phone numbers, birth dates, ethnicity and state of residence for an undisclosed number of job seekers and employers" (Acohido, 2009, p.3B). Reports of such breaches in data security could raise concern in the eyes of applicants who consider using the Internet to apply for jobs. This dissertation presents a timely investigation related to current events.

Job Relatedness and Invasiveness

As demonstrated by Rosenbaum's (1973) early study of privacy in personnel selection, one key variable in relation to privacy perceptions is the level of invasiveness that an individual perceives. In the context of personnel selection, Thibodeaux and Kudisch (2003) found that job relatedness was negatively related to invasiveness, leading them to assert that "selection tests that are perceived as face valid are generally viewed as non-invasive" (p.252). Several authors have focused on privacy perceptions in relation to 
specific test items (e.g., Sullivan \& Arnold, 2000). Organizations have the ability to choose the questions they ask in relation to test items. Still, some of the questions an employer must request from applicants are not job-related, but rather they asked due to federal regulation (Equal Opportunity Employment Commission, 2010). Therefore, depending on the questions posed to applicants, these individuals may perceive a selection procedure to be invasive. Though we know relatively little regarding perceived invasiveness and the construct's effect on more distal outcomes, we can gain insight from the work of Thibodeaux and Kudisch (2003). These researchers investigated the relationship between applicant reactions, perceived invasiveness, and perceived likelihood of legal claims with a real world sample of job applicants. Their findings indicated that participants who perceived the selection process to be invasive were more likely to believe that others would make a legal claim. Certain types of information disclosure may be viewed by an applicant more negatively than others. The effects of these negative perceptions may be demonstrated in applicant perceptions of justice.

As we are aware from organizational justice theory, the relevance of a particular procedure as it relates to the work an individual performs has been linked to individuals' perceptions of justice (Ambrose \& Alder, 2000). Therefore, providing detailed information about the use and security of personal information may prove to alleviate the negative effects the disclosure has on applicant reactions in this dissertation.

Furthermore, I propose that the concept of invasiveness is directly related to concerns regarding data security. In other words, applicants react to uncomfortable disclosures of personal information similarly to the way they react when they are concerned about data 
security. This is because in these situations applicants perceive that they are releasing personal information to entities with whom they do not wish to share it. Therefore, it is not only the content of the disclosure that frames the applicant reaction, but rather the perceived recipient of the personal disclosure.

\section{Control: Information Sharing and Privacy Concerns}

Stone and Stone (1990) cited "control" as a major component of the organizational privacy concept. Connerly et al. (2001) went a step further and applied "organizational privacy" to the selection context by proposing that applicants have a desire to control their personal information. Their proposal was a logical assertion that was consistent with other research, as individuals who use the Internet are often concerned with who can access their information and whether or not the information will be used inappropriately (Dinev \& Hart, 2004).

Control is a common theme in privacy research (Mael, 1998). For example, Dinev and Hart (2004) proposed that "Internet users are exposed to a risk of misuse and abuse of personal information that might raise the perception of vulnerability" (p.415). Indeed, these authors found that people who perceived that information submitted over the Internet was vulnerable were likely to be concerned with issues of privacy. In studies that deal with e-commerce, such as the aforementioned study, the privacy concerns of individuals are often focused on the misuse of personal information for purposes of marketing as well as identity theft (Dinev \& Hart, 2004). The latter appears to be more relevant to personal selection concerns, as well as general feelings of invasiveness. Unlike the privacy concerns that individuals may have when acting as consumers in an e- 
commerce context, job applicants may experience concerns related to questions typically asked of job applicants but not of consumers, such as Social Security Number.

Given that "personal information in a digital format can be easily copied, transmitted, and integrated ... " (Malhotra, Kim, \& Agarwal, 2004, p.336), it is reasonable to assume that job applicants who express concerns about personal information disclosure over the Internet may be apprehensive due to beliefs that their privacy may be compromised. Harris et al. (2003) supported this assertion when they found that reluctance to submit information over the Internet was related to job applicants' perceptions of privacy. Applicants may perceive risk when using Internetbased selection tools due to a belief that their personal information could be used inappropriately. Therefore, Stone and Stone's (1990) proposition that control can mitigate such beliefs is plausible; this dissertation proposed that perceived control over the sharing of information would mitigate high levels of privacy concerns such that overall perceptions of fairness of the Internet-based selection process would be increased.

\section{Privacy Context}

There are numerous differences between the consumer context found in much of the privacy concern literature and the employment context found in much of the organizational privacy literature. For example, several pieces of highly sensitive information are requested as a part of the employment application process that are not requested as a part of the purchasing process undertaken by a consumer. Data such as social security number, demographics, credit reporting, and welfare-to-work (incomerelated) are often requested in the United States by the Bureau of Labor Statistics and 
hence, are some of the types of information or releases for information requested by employers (Bureau of Labor Statistics, 2009). Therefore, a negative reaction by the job applicant may follow such requests due to applicants' concerns regarding data privacy. Privacy Policy in the United States

Bellman et al. (2004) described privacy regulation in the United States as "sectoral" in that it is aimed at specific businesses (mostly businesses that collect personal data from individuals and government entities), is undertaken voluntarily, and relies on consumers to file claims when they perceive wrongdoing. On the other hand, privacy regulation in the European Union is "omnibus" in that it is aimed at both public and private sectors, is aimed at all businesses, and is enforced under directive (e.g., Directive on Privacy and Electronic Communications, 2002).

In the United States, piece-meal court decisions stemming from civil lawsuits originating from individuals/groups of individuals have driven employment-related privacy policy rather than specific legislation. Several court cases have come about regarding applicant perceptions of invasiveness and privacy in regards to selection procedures. Though some do not involve Internet-based methods ( $\underline{\text { Soroka v. Dayton }}$ Hudson, 1991), such cases are worthy of attention because they give the community insight into the concerns of applicants in the United States. For example, one major court decision involved the invasiveness of items included in a personality-based selection test (Soroka v. Dayton Hudson, 1991). In this case, the plaintiff claimed that their personal privacy was invaded due to invasive questions included in the test, a measure of personality. On the other hand, the defendant argued that the test was a valid measure. 
The case was eventually settled out of court. This case demonstrates that questions for selection, whether statistically valid or not, could be considered by applicants as too invasive for use by organizations when selecting applicants.

To date, at least one court case in the United States has garnered attention regarding data integrity/protection and the selection of job applicants. A class-action lawsuit was filed against Aetna, an employer, in Pennsylvania District Court. The lawsuit claims that Aetna "allowed hackers to enter its Web site and gain access to Social Security numbers from about 65,000 current, former and potential employees" (Bordonaro, 2009, p.1). Though the outcome of this lawsuit remains pending, it is directly relevant to this dissertation. The measurement of privacy concerns in this study focused on concerns about data protection, data integrity, and the protection of personal information considered to be private (social security number, income, etc.). It also explored how such privacy concerns related to applicants' fairness perceptions. 


\section{Chapter 6:}

Hypotheses

The literature presented in the previous chapters of this dissertation supports a model of applicant reactions to Internet-based selection procedures presented in Figure 2. The model is unique in that it incorporates variables uniquely affected by the context of Internet-based selection but heretofore untested in the literature. Results obtained from testing this model are more up-to-date than the results presented by previous authors, as the use of the Internet has grown substantially in a short amount of time (Miniwatts Marketing Group, 2009). Privacy concerns were hypothesized to predict applicant fairness perceptions, which were proposed to result in distal applicant reactions including litigation intentions, purchasing intentions, job-acceptance intentions, recommendation intentions, and test-taking motivation. Moderators of these relationships were tested, to include applicants' perceived control of their private information and explanations provided to applicants about the Internet-based selection process. Finally, I proposed research questions that were designed to help explain differences in privacy concerns. Privacy Concerns

Privacy concerns are often related to fairness perceptions. For example, Eddy et al. (1999) demonstrated that employee privacy concerns were correlated with perceptions of fairness such that higher levels of privacy concerns were related to lower levels of perceived fairness. More specifically related to this study, Bauer et al. (2006) demonstrated that privacy concerns predicted fairness perceptions in the Internet-based 
selection context. Consistent with the results of these studies, this dissertation sought to replicate these findings by proposing that:

Hypothesis 1: Overall privacy concerns will predict fairness perceptions such that applicants with lower levels of privacy concerns will have significantly greater perceptions of fairness than applicants with higher levels of privacy concerns.

In order to better understand the individual dimensions related to the overall construct of privacy concerns, and thereby create a more comprehensive model, this dissertation proposed several hypotheses that sought to identify the relationships privacy concerns have with fairness perceptions. Whereas Harris and colleagues (2003) used a scale that lacked the statistical foundation on which to aggregate individual aspects of privacy concerns into an overall perception, this dissertation utilized a revised version of the "Concern for Information Privacy Scale" (Stewart \& Segars, 2002) that has previously demonstrated the multidimensional nature of privacy concerns. These dimensions include four factors: collection, improper access, errors, and unauthorized secondary use. Therefore, as an extension of the literature, the use of this scale allowed for the proposition of several hypotheses related to the individual dimensions of the privacy concerns construct such that:

Research Question 1: Will individual dimensions of privacy concerns predict fairness perceptions? Specifically, will participants with (a) greater concerns over data 
collection, (b) greater concern over improper access, (c) greater concern over errors, and (d) greater concern over unauthorized secondary use experience more negative overall perceptions of fairness?

\section{Explanations}

Providing explanations to applicants has been shown to elicit more positive reactions from job applicants (Truxillo et al., 2009). As demonstrated in previous research, informational "excuses" tend to be more powerful than "justifications" when providing explanations (Shaw et al., 2003), although this finding was not upheld in the Truxillo et al. meta-analysis of applicant reactions specifically. Furthermore, explanations that are perceived as adequate such that they are comprehensive are related to more positive perceptions of fairness (Shaw et al, 2003), although again, this has not been specifically examined in the applicant reactions literature. Consistent with the findings of Truxillo et al. (2002), explanations designed to address the specific concerns of applicant were expected to be related to higher levels of fairness perceptions. Given that privacy is proposed to be a key concern of applicants in the Internet-based selection context, I proposed that explanations would interact with privacy concerns such that they negative perceptions of privacy would be mitigated by explanations. Specifically, I proposed the following in order to advance our understanding of the effects of explanations on applicant reactions (Figure 2): 
Hypothesis 2: There will be a negative relationship between privacy concerns and fairness perceptions. However, this effect will be reduced by providing applicants with explanations. Specifically, privacy explanation will reduce this effect the most, followed by an excuse explanation.

Perceived Control over Personal Information

Eddy at al. (1999) demonstrated that control over an individual's personal information affected fairness perceptions when they found that individuals who had control over who could view or handle their information felt as though their privacy was less invaded and that the procedures used in reviewing their information were fair. In the present study, I manipulated control by allowing individuals to choose whether or not to release their personal identification number to the fictitious company to which the participant submits an application. Given the aforementioned previous findings that demonstrate control as moderating variable of perceived invasiveness and fairness perceptions, I expected these findings to translate to the Internet-based selection context. Therefore, this study sought to replicate the findings Eddy et al. (1999) and extend them to the selection context through which it was expected that (Figure 2):

Hypothesis 3: There will be a negative relationship between privacy concerns and fairness perceptions. However, this effect will be reduced by providing applicants with the opportunity to choose to release their personal information to the employer (control). 


\section{Fairness Perceptions}

As demonstrated in the review of literature presented in this dissertation, fairness perceptions are related to a variety of real-world outcomes that impact organizations. Per the event mediation approach to organizational justice (Ambrose et. al, 2007), the distal outcomes of an event or perception of an event is proposed to be mediated by a subsequent event. In the context of applicant reactions, the implication is that overall fairness perceptions will mediate the relationship between privacy perceptions and each distal outcome, rather than individual types of fairness perceptions (i.e., process fairness, outcome fairness) functioning to mediate the relationship between privacy perceptions and select distal outcomes.

Six distal outcomes were selected to be examined in this study for their propensity to affect organizations, including intentions to litigate, purchasing intentions, reapplication intentions, job acceptance intentions, recommendation intentions, and testtaking motivation. First, I proposed hypotheses regarding the relationships between privacy concerns, fairness perceptions, and ligation intentions (Hypothesis 4a, Hypothesis 5a). Previously, Bauer and her colleagues (2001) demonstrated that intentions to litigate were positively correlated with negative perceptions of fairness regarding the selection process in a procedure that involved participants who "imagined" applying for a job. This dissertation study employed a high fidelity application situation in order more accurately measure the relationship between litigation intentions and fairness perceptions while also determining whether or not privacy concerns specifically had an effect on such intentions. Second, I proposed hypotheses regarding the relationships between privacy 
concerns, fairness perceptions, and support for the organization through purchasing intentions (Hypothesis 4b, Hypothesis 5b). Aside from Macan et al.'s (1994) study that indicated that applicant reactions were related to consumer behavior, research on this outcome of fairness perceptions in the selection process is scant. This study sought to further our understanding of this variable while investigating the relationship purchasing intentions had with privacy concerns. Third, I proposed hypotheses regarding the relationships between privacy concerns, fairness perceptions, and reapplication intentions (Hypothesis 4c, Hypothesis 5c). Gilliland (2001) found that positive justice perceptions were related to increased reapplication behavior in a small sample of real-world applicants to a tenure-track professor position. This study sought to extend the generalizability of this finding by testing the proposed hypotheses with a larger sample of participants and measuring the effects of privacy concerns on this variable of interest. Fourth, I proposed hypotheses regarding the relationships between privacy concerns, fairness perceptions, and job acceptance intentions (Hypothesis 4d, Hypothesis 5d). Whereas Ployhart and Ryan (1998) found no relationship between fairness perceptions and job acceptance intentions, Smither et al. (1993), Macan et al. (1994), and a metaanalysis by Hausknecht et al. (2004) demonstrated positive relationships between applicant reactions variables (fairness perceptions) and job acceptance intentions. Therefore, this study sought to replicate the findings of Smither et al. and Macan et al. as well as test the relationship between privacy concerns and job acceptance intentions. Fifth, I proposed hypotheses regarding the relationships between privacy concerns, fairness perceptions, and recommendation intentions (Hypothesis 4e, Hypothesis 5e). 
Gilliland (1994) found a positive relationship between fairness perceptions and recommendation intentions in the selection context, which Hausknecht et al. (2004) further supported via a meta-analysis that demonstrated a strong relationship $(K=17, r=$ .52) between procedural justice perceptions, distributive justice perceptions, attitudes toward tests and the outcome variable of "recommendation intentions." Therefore, I proposed hypotheses consistent with these findings. Finally, I proposed hypotheses regarding the relationships between privacy concerns, fairness perceptions and test-taking motivation (Hypothesis 4f, Hypothesis 5f). Like Chan et al.'s (1997) findings that demonstrated a positive relationship between fairness perceptions (face validity) and testtaking motivation, I proposed similar hypotheses:

Hypothesis 4: Applicant reactions in the form of overall fairness perceptions will predict distal applicant reactions outcomes such that more positive overall perceptions of fairness will relate to (a) decreased intentions to litigate, (b) increased purchasing intentions, (c) increased intentions to reapply for positions with the same company, (d) increased job acceptance intentions, (e) increased willingness to recommend employment opportunities with the company to others (recommendation intentions), and (f) higher levels of test taking motivation.

Hypothesis 5: Applicant reactions in the form of overall fairness perceptions will mediate the relationship between privacy perceptions and each distal applicant reactions such that more positive overall perceptions of fairness will relate to (a) decreased intentions to 
litigate, (b) increased purchasing intentions, (c) increased intentions to reapply for positions with the same company, (d) increased job acceptance intentions, (e) increased willingness to recommend employment opportunities with the company to others (recommendation intentions), and (f) higher levels of test taking motivation.

\section{Exploratory Research Questions}

Perceived Belief in Legal Protection

Common knowledge informs researchers that applicants may have varying levels of belief in the protections offered by the law. Specifically, applicants using Internetbased selection process may have varying levels of concern with providing personal information using the Internet due to their beliefs that the law protects them. Therefore, belief that legal protection exists may influence individuals' privacy concerns. Hence, I asked:

Research Question 2: Will the perceived belief in legal protection predict overall privacy concerns such that participants with stronger beliefs that the law protects them will have less privacy concerns? 
Chapter 7:

Method

\section{Design}

This dissertation study used a 3 (No Explanation/Excuse Explanation/Privacy Justification Explanation) X 2 (Control/No Control) between subjects factorial design. Over 600 students were invited to participate from classes at two post-secondary institutions located in the Pacific Northwest and Central Florida regions of the United States. Students were invited to participate in the proposed study through a recruitment form received by the participant via email invitation (Appendix B). The process participants' followed is outlined in Appendix K. First, each participant proceeded to a website to participate in the study by entering a web address provided in the invitation email in their web browser. Participants were permitted to use any computer to which they had access. This is similar to Internet-based selection procedures used in real-world contexts, as applicants are often allowed to apply for positions using personal computers, computers at public libraries, etc. The first page of the website contained informed consent (Appendix C) and an introduction to the study that provided a brief description of the purpose of the study (Appendix D). Participants were informed that they would receive extra credit points for participation. Participants were also informed of a $\$ 100$ prize for the participant who would be selected for the position with the fictitious company (The Smith Company). The prize was intended to provide participants with motivation to take the selection procedure seriously, so as to create a more high fidelity test-taking situation. Next, participants completed pre-test measures, which were similar 
to the post-test measures (Appendix A). Results from the pre-measures were evaluated for use as controls. Then, participants proceeded to the fictitious 20-minute Internetbased application that included demographic information and an Internet-based selection test that consisted of personality-based items focused on measuring conscientiousness (Appendix E). One key attribute of the Internet-based selection test was that it requested a portion of the participant's Social Security Number (the last four digits), which was designed to heighten the test's invasiveness, such as in a real-world selection test. During the Internet-based selection test, each participant was randomly assigned and exposed to one of six conditions, as outlined in Table 1. Participants viewed the excuse explanation (Appendix F), the privacy justification explanation (Appendix G), or no explanation (Appendix $\mathrm{H}$ ) at the beginning of the fictitious application process. Then, on the last page of the fictitious application process, participants were exposed to either the control (Appendix I) or no control (Appendix J) condition. Following completion of the Internetbased application, each participant received a notification of rejection in the form of a letter that restated the explanation condition experienced before the fictitious application process (Appendix L, Appendix M, or Appendix N). All participants "failed" the selection process, as this is the likely outcome for most job applicants in real-world contexts. In addition, rejected applicants are those who are likely to react most negatively and for whom explanations and control were expected to have the strongest effects. Next, participants responded to a self-report post-test questionnaire (Appendix A) that assessed participants' privacy concerns, perceived belief in legal protection, fairness perceptions, job acceptance intentions, purchasing intentions, litigation intentions, 
recommendation intentions, and test-taking motivation. Finally, participants were debriefed one week later in a thank-you letter that exposed the deception used in the study and reassured participants about the protection of their personal data (Appendix O). Participants

Participants were recruited from multiple classes at post-secondary institutions in the Pacific Northwest (Portland State University in Portland, Oregon) and Central Florida (Florida Technical College in Orlando, Florida) regions of the United States. Incentives were provided to study participants in the form of extra-credit points applied to the course grade, and one participant was selected at random to receive a $\$ 100$ prize (Appendix $\mathrm{P}$ ).

Power Analysis. To date, no single method is recommended in the literature to make an a priori prediction for a desired sample size in a study that proposes a model that includes both mediation and moderation with categorical variables. Still, calculations can be used that are based on previous effect sizes for the distal outcome variables measured in this study. Such effect sizes range from .02 (reapplication intentions) (Ployhart \& Ryan, 1998) to .52 (recommendation intentions) (Hausknecht et al., 2004). Furthermore, Truxillo et al. (2009) meta-analysis found an effect size of .09 for the relationship between explanations and fairness perceptions (procedural justice). Given the wide range in effect sizes, a moderate approach to conducting power analysis involved using a reasonable effect size of $.20\left(f^{2}=.04\right)$. Upon using $\mathrm{G}^{*}$ Power, a statistical program for power analysis, (Faul, Erdfelder, Buchner, \& Lang, 2009), the estimated minimum sample size for the prediction of significant outcomes for the mediated portion of the proposed model, given .80 power, was found to be 304 participants. In order to determine 
the estimated minimum sample size for the prediction of outcomes for the moderated portion of the model, I used previous work by Aguinas, Beaty, Boik, and Pearce (2005). These authors suggest that the sample size for a model that contains categorical moderation and expects an effect size of $.20\left(f^{2}=.04\right)$ would be mostly likely 293 participants in previous research (p.99). Given these calculations, I expected that I would need 300 participants to participate in the study in order to achieve adequate power.

\section{Measures: Antecedents}

A complete list of measures is found in Table 2.

Privacy concerns. Previously, scales to measure the privacy concerns of job applicants were developed by authors using intuitive development of items with little evidence of statistical validity. For example, Harris et al. (2003) created a twelve-item scale that demonstrated correlations between individual items and relevant outcomes but did not appear valid when used as an overall measure of privacy concerns. Specifically, the scale lacks utility because it should not be used as a single measure of privacy perception because the "pattern of results differed from item to item" (p.232). Therefore, this dissertation used the Stewart and Segars (2002) "Concern for Information Privacy" (CFIP) scale, which was later adapted by Bellman et al. (2004) in order to fit the Internet context (Appendix A). Originally developed to measure the privacy concerns of consumers, the CFIP instrument was created using relevant theory and commonly accepted statistical procedures (i.e., Confirmatory Factor Analysis). Research by Stewart and Segars (2002) indicated the multidimensionality of privacy concerns, with collection, improper access, errors, and unauthorized secondary use being individual components 
with both first-order and second-order validity. In other words, both the individual factors and overall scale score demonstrate validity in predicting behavioral intentions (support for the organization). An example item from the adapted scale reads "Web sites should devote more time and effort to preventing illegal access to personal information." The authors report that this revised 15-item scale previously demonstrated internal consistency that was close-to or above $\alpha=.70$ (Bellman et al., 2004). Given the low internal consistency of the scale, this dissertation also investigated how each subscale or dimensions of privacy concerns predicted fairness perceptions (RQ1). The internal consistency of the overall scale, as used in the present study, was acceptable $(\alpha=.91)$. Internal consistencies for the collection factor $(\alpha=.91)$, the errors factor $(\alpha=.85)$, the unauthorized secondary use factor $(\alpha=.91)$ and the improper access factor $(\alpha=.92)$ were also acceptable in this study. Timing of the scale was both pre-test and post-test in the pilot study, but post-test only in the main study due to effects of the pre-test CFIP measure described in Chapter 8: Results.

Belief in legal protection. Participants' beliefs that they are protected from possible harm related to the use of the Internet-based selection procedure will be measured with three items created for the purpose of this study. An example item reads "I believe that laws and regulations protect my privacy." Internal consistency estimates were obtained during the proposed pilot but no changes were made based on findings. Timing of the scale was both pre-test and post-test in order to establish a baseline measure to compare with post-test perceptions. The internal consistency of this scale, as used in the present study, was acceptable $(\alpha=.93)$. 
Selection procedural justice. Fairness perceptions were measured using several scales included in the overall Selection Procedural Justice Scale (SPJS) developed by Bauer and colleagues (2001). This scale was created through a rigorous five-phase development process to provide a valid measure of justice consistent with the procedural justice rules outlined by Gilliland (1993). The SPJS is comprised of three high-order factors: the Social Factor $(\alpha=.93)$, the Structure Fairness Factor $(\alpha=.88)$, and the JobRelatedness-Content Factor $(\alpha=.89)$. Scales measuring lower-order dimensions within the Structure Fairness Factor (job-relatedness-predictive, information known, chance to perform), Social Fairness Factor (propriety of questions) and the Job-Relatedness Content Factor (job-relatedness-content) will be used. An example item reads "The content of the test did not appear to be prejudiced." Bauer et al. reported an overall internal consistency of $\alpha=.78$ for the SPJS. The internal consistency of the overall scale, as used in the present study, was acceptable $(\alpha=.90)$. Timing of the scale was post-test.

Overall perceptions of procedural justice (Fairness). In addition to the use of the SPJS, two items from Thibaut and Walker (1975) and four items from Leventhal (1980) were used to measure overall perceptions of procedural justice, consistent with Colquitt's (2001) use of these items, who found an internal consistency of $\alpha=.78$ for the combined use of these items. An example item reads "I had influence over the hiring decision that was made based on the Internet-based selection process." The internal consistency of this scale, as used in the present study, was acceptable $(\alpha=.86)$. Timing of the scale was post-test. 
Distributive justice. Four items from Leventhal (1976) were adapted and used to measure overall perceptions of distributive justice in the post-test measures, consistent with Colquitt's (2001) use of these items, who found an internal consistency of $\alpha=.92$. An example item reads "The hiring decision reflects the effort I put into the Internetbased selection process." The internal consistency of this scale, as used in the present study, was acceptable $(\alpha=.91)$. Timing of the scale was post-test.

\section{Measures: Outcome Variables}

Recommendation intentions will be measured with a three-item scale used in previous research by Bauer et al. (2001). An example item reads "I intend to encourage others to apply for a job with this company." This scale previously demonstrated high internal consistency consistency $(\alpha=.95)$ in the Bauer et al. study. The internal consistency of this scale, as used in the present study, was acceptable $(\alpha=.89)$. Timing of the scale was post-test and pre-test in order to determine change in behavioral intentions related to participation in the study.

Reapplication intentions were proposed to be measured with two items, one item adapted from Ployhart and Ryan (1997) that read "I would apply to a job with [company name] again" and a second item created by the author. Upon construction of the procedure in the web-based survey tool, the items for reapplication intentions were inadvertently omitted. Thus, reapplication intentions were not explicitly measured in this study, although it is likely that this behavioral intention would have functioned similar to those within the "Organizational Intentions" variable created through Confirmatory Factor Analysis in Chapter 8: Results. 
Job acceptance intentions were measured with a two-item scale based on that used in previous research by Ployhart and Ryan (1997). An example item reads "If selected, I intend to accept a position with [company name].” Ployhart and Ryan reported a high internal consistency of $\alpha=.98$. The internal consistency of this scale, as used in the present study, was acceptable $(\alpha=.98)$. Timing of the scale was to be post-test.

Purchasing intentions were measured by a three-items. Two items were created by the author for use in this study, and one item was used previously in research by Macan et al. (1994). An example item item reads "I would shop at a store owned by The Smith Company." The internal consistency of this scale, as used in the present study, was acceptable $(\alpha=.83)$. Timing of the measure was post-test and pre-test in order to determine change in behavioral intentions related to participation in the study.

Litigation intentions were measured by an adapted four-item scale used previously in the Bauer et al. (2001) study. An example item reads "An organization that uses an Internet-based application and test like this would likely be sued by applicants." Bauer and colleagues found high internal consistency for this measure $(\alpha=.91)$. The internal consistency of this scale, as used in the present study, was acceptable $(\alpha=.94)$. Timing of the measure was post-test and pre-test in order to determine change in behavioral intentions related to participation in the study.

Test-taking motivation was measured using the 10-item Test-taking Motivation, Valence, Instrumentality, and Expectancy Motivation Scale (VIEMS) developed and validated by Sanchez et al. (2000). An example item reads "I would like to be hired for this job." This overall scale previously demonstrated moderately high internal 
consistency $(\alpha=.80)$ in the Sanchez et al. study. Internal consistency reliabilities for the valence $(\alpha=.95)$, instrumentality $(\alpha=.84)$, and expectancy $(\alpha=.82)$ subscales were also satisfactory. The internal consistency of the overall scale, as used in the present study, was acceptable $(\alpha=.93)$. Timing of the measure was both pre-test and post-test as preand post-testing motivation have been found to be qualitatively different (Chan et al., 1997).

\section{Measures: Potential Control Variables}

Internet experience and knowledge of the Internet were measured as control variables in order to reduce the likelihood of a confounding effect on study results. Specifically, participants were asked to self-report their perceived experience level with the Internet as well as indicate their perceived knowledge of the Internet with two items using responses on a scale of one to ten with ten being the highest rated level of experience and/or knowledge. Timing of the scales was post-test.

Electronic application preference (Internet vs. Paper-basedl) was measured by three items developed by the author for the purpose of the proposed study. An example item reads "I would prefer to complete an Internet-based job application rather than a paper- based job application." The internal consistency of this scale, as used in the present study, was acceptable $(\alpha=.96)$. Timing of the scale was both pre-test and posttest.

Demographic variables including age, ethnicity, and gender were collected via the Internet-based selection process which volunteer applicants (participants) used in order to participate in the study. Demographic variables including employment status and 
time elapsed during job search were collected via questions created by the author. The relationships between these variables and privacy concerns were explored. Demographics that have a consistent relationship with the dependent variables were considered as control variables (See Results).

\section{Manipulations}

Explanations/Information was manipulated such that participants will be placed in one of three conditions. In the "excuse explanation" condition (Appendix F; Appendix L) an excuse was provided for the use of the Internet-based selection process. An excuse explanation was used because it had been shown to be the most effective explanation type (Gilliland, 2001). In the "privacy justification explanation condition” (Appendix G; Appendix M) the use of the Internet-based application was justified using an explanation that specifically addresses privacy concerns. Though Gilliland found that justifications were not as effective in eliciting positive applicant reactions from participants as excuses, it was hypothesized that in the Internet-based selection context the privacy justification explanation condition would be more effective (H3). In the no explanation condition (Appendix H; Appendix I) there was an absence of an explanation provided to applicants regarding the use of an Internet-based selection process.

Control was manipulated such that participants were placed in a "control over information" condition versus a "no control over information" condition. The control over information condition (Appendix I) provided participants with the opportunity to release their personal information to the employer. The no control over information condition (Appendix J) did not afford such an opportunity to participants. 
Manipulation check items were used to determine whether a participant was paying adequate attention to the Internet-based selection procedure. Three items were used to determine the salience of the experimental conditions to participants. One item read "An explanation was provided to me regarding the purpose or use of the selection procedure" and a second item read "I had the opportunity to decline the release of my personal information to The Smith Company." A third item was used to be sure the participant was aware of rejection and read "I was not offered a job with the Smith Company." 


\section{Chapter 8:}

Results

\section{Conditions}

Table 1 outlines the multiple conditions to which participants were exposed during the study. As previously stated, a manipulation check was used to identify whether study participants were aware of the manipulations in the study.

\section{Pilot Study}

A pilot study targeting 50 participants was conducted for two reasons. First, the pilot study aimed to assess the study participant completion rate. Second, the pilot study sought to determine if there were priming effects associated with the administration of the Concern for Information Privacy Scale (CFIP) both pre-test and post-test versus solely post-test administration.

A total of 52 participants agreed to participate in the study. Of the 52 participants who began the survey, 5 abandoned the survey after agreeing to participate, 47 completed only the pre-test measures, 41 completed the pre-test measures and at least a portion of the Internet-based selection procedure/test, and 39 completed the pre-test measures, at least a portion of the test, and at least a portion of the post-test measures. Therefore, the overall number of pilot study participants was 39, establishing a completion rate of $65 \%$. Means, standard deviations, internal consistencies, and intercorrelations for the pilot study variables are found in Table 3 .

Aside from determining whether the procedure would work with actual participants, the second reason for the pilot was to determine whether or not the Concern 
for Information Privacy Scale (CFIP), when administered pre-test and post-test, would "prime" the responses of participants. Therefore, the CFIP was administered both before and after the test for half of the pilot participants. The other half of the pilot participants only received the CFIP at the post-test. I used Analysis of Variance (ANOVA) to determine if the two groups differed significantly in their responses to dependent variables of interest in this study, including justice perceptions.

The one-way ANOVA indicated that there were significant differences in the responses of individuals who received the CFIP both pre-test and post-test as compared to those who received the CFIP only post-test when responding to a key scale in my study-- the distributive justice scale $F(1,36)=107.734, p<.01$, such that participants who received the CFIP at both pre-test and post-test timing had significantly higher perceptions of distributive justice $(M=2.79, \mathrm{SD}=.96)$ than those who received the CFIP only at post-test timing $(M=1.94, \mathrm{SD}=.91)$. Given this finding, the pre-test CFIP measure was eliminated, which may have also helped to increase the study completion rate, because this difference suggests that the pre-test CFIP could act as an intervention in itself and affect participant responses.

\section{Data Preparation}

Data from the 18 participants who received the CFIP at the post-test only timing during the pilot study were combined with the data from the 472 individuals who participated in the primary study, equaling a total of 490 participants. The dataset was then cleaned using a three-step process that included a visual scan for bogus data, the examination of the manipulation check, and the use of elimination criteria based on study 
completion. Participants included in the final dataset had to have completed at least a portion of the pre-test measures, a portion of the selection procedure, and a portion of the post-test measures.

First, the dataset was visually scanned by the author in order to determine if there were any bogus data. "Bogus data" were regarded as cases where participants responded the same to every question or appeared to enter data in a suspicious pattern (e.g., everyother question was rated the same). This visual scan for bogus data eliminated 8 participants during the data cleaning process. Next, responses to the three manipulation check items were examined (Appendix A). For analysis purposes, one point was assigned per manipulation check question correctly answered by each participant. Participants who did not answer at least two questions correctly were eliminated. Based on these criteria, 65 participants were removed from the dataset. Finally, a standardized set of elimination criteria was applied to each remaining participant. In order to be included in the final dataset, the remaining participants had to have (a) completed at least one scale in the premeasures in entirety, (b) had to have responded to at least one item in the selection procedure that could be perceived as invasive by the applicant (i.e., provided the partial social security number, responded to a demographic question, or responded to more than one personality item), and (c) had to have responded to at least one scale in entirety in the post-test measures section. Based on this final test of inclusion, 37 participants were removed from the dataset.

Given that privacy concerns are of central concern to this study, it was important to determine if participants who were eliminated due to a lack of completion were 
somehow significantly different from those who completed the study. Specifically, those who did not complete the study could have been concerned about the implications of the study, thereby ceasing participation, and restricting the range of study participants such that only those with the most positive reactions participated. In order to determine whether or not the 37 excluded participants were different from those who were included, several $t$-tests were performed on the pre-test variables as well as a visual review of the excluded participant data. Excluded participants had significantly higher recommendation intentions $(M=3.93, \mathrm{SD}=.75)$ than included participants $(M=3.61, \mathrm{SD}=.85)$, significantly lower job acceptance intentions $(M=4.36, \mathrm{SD}=.76)$ than included participants $(M=4.47, \mathrm{SD}=.73)$, and significantly higher litigation intentions $(M=2.70$, $\mathrm{SD}=1.09)$ than included participants $(M=2.26, \mathrm{SD}=.78)$. Excluded participants did not differ significantly on the other pre-test measures. Additionally, the visual examination of the excluded participants' data did not reveal any patterns or commonly avoided items.

\section{Sampling}

Given that participants in this study came from two geographically different areas (Central Florida and the Pacific Northwest), it was important to determine if the two samples were equivalent. Therefore, ANOVAs were conducted to determine if the samples differed on key variables (Table 4). ANOVAs revealed that differences between samples existed such that the Central Florida Sample differed significantly from the Pacific Northwest Sample among outcome variables such as test-taking motivation $F(1,371)=18.85, p<.01$, recommendation intentions $F(1,375)=22.22, p<.01$, purchasing intentions $F(1,375)=18.715, p<.01$, procedural justice $F(1,368)=73.36, p$ 
$<.01$, and distributive justice $F(1,377)=69.47, p<.01$ (Table 3 ). It was noted that participants from the Pacific Northwest had lower test taking motivation $(M=3.57, \mathrm{SD}=$ .89), lower purchasing intentions $(\mathrm{M}=3.49, \mathrm{SD}=.85)$, and lower recommendation intentions $(M=3.25, \mathrm{SD}=1.06)$ than participants from Central Florida $(M=4.13, \mathrm{SD}=$ $.96 ; M=3.94, \mathrm{SD}=1.04 ; M=3.99, \mathrm{SD}=.86)$. Additionally, participants from the Pacific Northwest had lower procedural justice perceptions $(M=2.45, \mathrm{SD}=.75)$ and distributive justice perceptions $(M=2.17, \mathrm{SD}=.92)$ than participants from Central Florida $(M=3.40, \mathrm{SD}=1.02 ; M=3.28, \mathrm{SD}=1.22)$.

The significant differences between the Pacific Northwest sample and the Central Florida sample on key variables of interest presented an issue when moving forward with the data analysis in the present study. Although sample origin could be controlled for in hierarchical regression analysis, the differences between samples would present problems when confirming the factor structure of the scales used in this study. Providing support for rigorous measures of psychological constructs was central to testing hypotheses. Therefore, measures that had not previously been used in investigations of applicant reactions (i.e., the Concern for Information Privacy Scale) and measures that may be best conceptualized as a single concept (i.e., recommendation intentions, purchasing intentions, and job acceptance intentions), were lent support by establishing factor structures through Confirmatory Factor Analysis (CFA). The substantial differences in group means between the two samples would cause problems with error variance when conducting a CFA. 
In addition to issues with combining the samples for the purposes of the CFA, the relatively large size of the Pacific Northwest sample in comparison to the Central Florida sample provided more rationale by which to eliminate the Central Florida sample. As outlined in Chapter 8: Method, a sample of approximately 300 participants was required per the number of variables and the expected effect sizes. Therefore, participants from the Central Florida sample were eliminated from the dataset and participants from the Pacific Northwest were included in the final dataset in order to ensure that statistical analysis could be performed.

Study Participants

Following the elimination of the Central Florida sample, 315 participants remained. The demographics of these participants, all from the Pacific Northwest, were examined. The mean age of participants was $25.36(\mathrm{SD}=7.38)$, and they had over 3 years of college education. Participants identified themselves mostly as female $(70.2 \%)$, mostly White/Caucasian (70.8\%), and mostly psychology students (90.5\%). Roughly one-third (33\%) of the participants were "currently seeking jobs" at the time of participation in the study. Most participants chose to complete the Internet-based selection procedure at home $(71.4 \%)$ or at work or school $(22.5 \%)$. Identification of Control Variables

Gender. Significant differences did not exist between males and females in responses to any of the variables of interest in this study. Therefore, gender is not used as a control variable in the present study. 
Ethnic background. Due to small sample sizes for ethnic background variables, several ethnic background groups were collapsed before analysis into a dichotomous variable. American Indian/Native American $(n=0)$, Asian $(n=26)$, Black/African American $(n=5)$, Latino/Hispanic $(n=22)$, Pacific Islander $(n=3)$, and two or more ethnicities $(n=15)$ were transformed into a dichotomous variable. This new variable for ethnic background was comprised of two groups where participants were identified as either White/Caucasian $(n=223)$ or Non-White/Non-Caucasian $(n=71)$. Following the collapse of several variables that indicated participants' ethnicities, ANOVA was used to examine possible significant differences in responses to variables of interest in this study including fairness (procedural and distributive justice) and the distal outcome variables (test-taking motivation, recommendation intentions, purchasing intentions, job acceptance intentions, and litigation intentions). White/Caucasian participants differed from Non-White/Non-Caucasian participants on one variable of interest - litigation intentions $F(1,292)=11.16, p<.01$ (Table 5), such that White/Caucasian participants had lower litigation intentions $(M=2.38, \mathrm{SD}=.95)$ than Non-White/Non-Caucasian participants $(M=2.83, \mathrm{SD}=1.06)$. Given this significant difference on a key variable of interest in this study, ethnic background is used as a control variable in the present study.

Electronic application preference. The preference for electronic applications was correlated with several variables of interest in this study, but correlations were mostly weak and with distal outcome variables (Table 5). Specifically, significant correlations were found with test-taking motivation $(r=.13, p<.05)$, litigation intentions $(r=-.12, p$ $<.05)$, recommendation intentions $(r=.13, p<.05)$, job acceptance intentions $(r=.17, p$ 
$<.01)$, and purchasing intentions $(r=.19, p<.01)$. Electronic application preference was not significantly correlated with procedural justice $(r=.04, p=.44)$ or distributive justice $(r=.00, p=95)$. Given these weak correlations, as well as the fact that the scale has not been used in previous research, electronic application preference was not used as a control variable in this study.

Internet knowledge \& Internet experience. Internet knowledge and Internet experience, as measured in this study, were highly correlated $(r=.76, p<.01)$. The high correlation between these two variables indicated that one of the variables, rather than both, could be used as a control, so as to minimize the degrees of freedom used in subsequent analyses. Theoretically, participants may have responded with more thought when responding to the Internet-experience item, as this variable may have been more salient to participants in this study. This may have been due to the fact that participants were not only using the Internet to complete the study, but also answering questions about their experiences with the Internet (e.g., CFIP scale). Furthermore, significant correlations between Internet experience and distributive justice $(r=.12, p<.05)$, testtaking motivation $(r=.13, p<.05)$, recommendation intentions $(r=.13, p<.05)$, and purchasing intentions $(r=.12, p<.05)$, indicated that using the Internet experience measure as a control variable would be most prudent.

\section{Scale Structure}

Confirmatory Factor Analysis was used to assess the structure of several scales used in this study. The distal outcome scales used to measure test-taking motivation (VIEMS), litigation intentions, recommendation intentions, job acceptance intentions, 
and purchasing intentions were entered into three models to obtain clean factor structures for three factors including test-taking motivation (Figure 7), organizational intentions (comprised of recommendation intentions, job acceptance intentions, and purchasing intentions) (Figure 8), litigation intentions (Figure 9), and CFA was also used to assess the structure of the fairness perceptions variable (Figure 10,11) and the Concern for Information Privacy (CFIP) scale (Figure 12).

First, test-taking motivation, as measured by VIEMS scale, was expected to be comprised of three factors as found in previous scale-development research (Sanchez et al., 2000). CFA was conducted using both a first order and second order factor structure (Figure 7). The second order factor was named “Test-Taking Motivation,” which predicted three first-order factors that measured "Valence," "Instrumentality," and "Expectancy." This structure was consistent with that described by Sanchez et al. (2000). Chi-Square results were significant $\left(\chi^{2}=241.60, d f=87, p=.00\right)$, but values for the Goodness of Fit Indices were close 1.00, indicating an acceptable fit, with NFI $=.93$, RFI $=.91$, and $\mathrm{CFI}=.96$. The RMSEA value was also below .10 , with $\mathrm{RMSEA}=.08$. Based on these results, the test-taking motivation scale was used as originally intended in this study. Coefficient alpha for the overall scale was $\alpha=.93$.

A second CFA was used to determine the fit of a three first-order factor model predicted by a second-order factor named "Organizational Intentions" (Figure 8). Organizational Intentions was believed to be comprised of first order factors that were comprised of the recommendation intentions scale (3 items), the job acceptance intentions scale ( 2 items), and the purchasing intentions scale ( 3 items). Chi-Square 
results were significant $\left(\chi^{2}=40.20, d f=17, p<.01\right)$, but values for the Goodness of Fit Indices were close 1.00 , indicating an acceptable fit, with NFI $=.99, \mathrm{RFI}=.97$, and CFI $=.99$. The RMSEA value was also below .10 , with RMSEA $=.07$. Based on these results, an "Organizational Intentions" variable was created through the addition of the recommendation intentions scale, the job acceptance intentions scale, and the purchasing intentions scale. This new variable was used in subsequent analyses. Coefficient alpha for this new scale was $\alpha=.91$.

The final distal outcome scale measuring litigation intentions was analyzed using CFA with a single factor "Litigation Intentions" predicting the four litigation intentions items (Figure 9). Chi-Square results were non-significant $\left(\chi^{2}=5.82, d f=2, p=.06\right.$ ), and values for the Goodness of Fit Indices were close 1.00, indicating an acceptable fit, with $\mathrm{NFI}=1.00, \mathrm{RFI}=.99$, and $\mathrm{CFI}=1.00$. The RMSEA value was also below .10 , with RMSEA $=.08$. Based on these results, the litigation intentions scale was used as it was originally intended in this study. Coefficient alpha for this scale was $\alpha=.94$.

As presented in Chapter 7, three measures of fairness perceptions were used in this study, including a measure of procedural justice (Leventhal, 1980; Thibaut \& Walker, 1975), a measure of distributive justice (Leventhal 1976), and selected items from the Selection Procedural Justice Scale (Bauer et al., 2001). After using Exploratory Factor Analysis to review the factor structure of the partial SPJS used in this study, it was clear that the abridged version of the measure did not reflect the three-factor structure of the original scale. Many individual items demonstrated by Bauer et al. 2001 to cluster with certain factors failed to align with those factors. For example, the item that read "the 
content of the test did not appear to be prejudiced" loaded on the Structure Fairness Factor rather than the Social Fairness Factor, and the item that read "I knew what to expect on the test" loaded on the Social Fairness Factor rather than the Structure Fairness Factor. Given these findings, the SPJS was excluded, and CFA was used to determine the factor structure of an Overall Fairness Measure comprised of items from the procedural justice and distributive justice scales. Previous research demonstrated that multiple justice variables function as one variable in the prediction of distal outcome variables (Colquitt, 2001). Therefore, procedural justice items and distributive justice items were placed into a factor model where the first-order factors of "procedural justice" and "distributive justice" were predicted by a second-order overall "Fairness" factor (Figure 10). Results indicated poor model fit, as the Chi-Square results were significant $\left(\chi^{2}=\right.$ 299.40, $d f=43, p=.00$ ), and values for the Goodness of Fit Indices were NFI $=.85$, RFI $=.78$, and CFI $=.87$. The RMSEA value was also above $.10($ RMSEA $=.14)$.

Given the poor fit of the initial model, modification indices were used to obtain a model with better fit (Figure 11). Correlations between error terms for the fourth procedural justice item and the fifth and seventh procedural justice items, the error terms of the fifth procedural justice item and the seventh procedural justice item, and the error terms of the first and second distributive justice items were added to the model. With these modifications, the resulting model achieved better, acceptable fit. Though the ChiSquare results were significant $\left(\chi^{2}=139.17, d f=39, p=.00\right)$, values for the Goodness of Fit Indices were acceptable at NFI $=.95, \mathrm{RFI}=.90$, and CFI $=.95$. The RMSEA value was also below $.10($ RMSEA $=.09)$. Based on these results, an "Overall Fairness 
Perceptions" was created via the addition of the procedural justice and distributive justice scale scores and subsequently used as the mediating variable in the proposed model. Coefficient alpha for this overall justice scale was $\alpha=.90$.

Another scale of interest in this study was the Concern for Information Privacy Scale (CFIP) which was purported to be comprised of a 4 factors including collection, improper access, errors, and unauthorized secondary use. Though the majority of analyses in this study use the CFIP as an overall measure of privacy concerns, one hypothesis proposed that the individual dimensions of the CFIP may differentially predict fairness perceptions. As such, Confirmatory Factor Analysis was used to determine if there were four separate factors contributing to Privacy Concerns. CFIP items were placed into a factor model where the first-order factors of "collection," "improper access," "errors," and "unauthorized secondary use" were predicted by a second-order overall "Privacy Concerns" factor (Figure 12) and analyzed for goodness of fit. Based on results, this model had good fit. Though the Chi-Square results were significant $\left(\chi^{2}=\right.$ 171.32, $d f=84, p=.00)$, values for the Goodness of Fit Indices were acceptable at NFI = $.95, \mathrm{RFI}=.93$, and CFI $=.98$. The RMSEA value was also below $.10(\mathrm{RMSEA}=.06)$. Therefore, the individual dimensions could be used as separate predictors of fairness during hypothesis testing. Coefficient alphas for each of the CFIP subscales as well as the overall subscale are presented in Table 7.

Following the findings of the CFAs, the proposed model was refined as demonstrated in Figure 3. As shown in the figure, Hypotheses 4a and 5a remained unchanged. Hypotheses 4b, 4c, 4d, along with Hypotheses 5b, 5c, and 5d, were collapsed 
into Hypothesis $4 \mathrm{~b}$ and Hypothesis $5 \mathrm{~b}$ in order to reflect the new "Organizational Intentions" variable. Hypotheses 4e and 5e were reindentified as Hypotheses 4c and 5c. Scale Analysis

Reliabilities, means, and intercorrelations for study scales, including the revised scales for "Organizational Intentions" and "Overall Fairness Perceptions," can be found in Table 6. As demonstrated in the table, scale internal consistency estimates all demonstrated good internal consistency with all study scales achieving $\alpha>.80$. Furthermore, scale means for fairness perceptions were below 3.00, as expected in a study where all participants receive a negative notification of rejection.

\section{Hypothesis Tests}

Hypothesis 1, Hypothesis 2, and Hypothesis 3 were tested using hierarchical regression. Hypothesis 1 posited that overall privacy concerns would predict fairness perceptions such that applicants with lower levels of privacy concerns would have significantly greater perceptions of fairness than applicants with higher levels of privacy concerns. Hypotheses 2 and 3 sought to determine if two variables (i.e., the study conditions), explanations (H2) and control (H3), moderated the relationship between overall privacy concerns and overall fairness perceptions. As such, control variables (i.e., Dichotomous Ethnicity, Internet Experience) were entered into the first step of the regression equation. Then, dummy-coded study conditions (i.e., Control vs. No Control, Excuse Explanation vs. No Explanation, Privacy-Justification Explanation vs. No Explanation,) were entered into the second step of the regression equation. Third, overall privacy concerns were entered into the regression equation. Finally, the interaction 
between the control condition and privacy concerns, as well as the interactions between the explanation conditions and privacy concerns, were entered into the fourth and final step of the regression equation. The overall model was significant $F(9,271)=2.36, p<$ .05 with a significant $R^{2}$ change between the second and third models, but no significant change in $R^{2}$ in the final model. Results are shown in Table 8.

As can be seen in Table 8, results of the hierarchical regression for Hypotheses 1 , 2 , and 3 indicated that overall privacy concerns was a significant predictor of fairness perceptions such that higher overall privacy concerns predicted lower overall fairness perceptions in the third regression equation $(\beta=-.14, t=-2.43, p<.05)$, with a significant amount of variance uniquely predicted by privacy concerns $\left(\mathrm{R}^{2}\right.$ change $=.02, \mathrm{p}$ $<.05)$, in support of Hypothesis 1 . The main effects of control $(\beta=.11, t=1.78, p=$ $.08)$, the excuse explanation $(\beta=.05, t=.67, p=.50)$, and the privacy-justification explanation $(\beta=.11, t=1.66, p=.10)$ on fairness perceptions were not significant in the second regression equation. The main effects of control $(\beta=.11, t=1.89, p=.06)$, the excuse explanation $(\beta=.05, t=.75, p=.46)$ and the privacy-justification explanation $(\beta$ $=.10, t=1.47, p=.14$ ) on fairness perceptions were not significant in the third regression equation, although it is important to note that the effects of control approached significance in this third step. Lastly, the fourth and final regression equation was examined in order to determine if control and explanations served as moderators of the privacy concerns-fairness perceptions relationship. The interactions between privacy concerns and control $(\beta=.05, t=.10, p=.92)$, between privacy concerns and excuse explanations $(\beta=-.47, t=-.84, p=.40)$, and between privacy concerns and privacy- 
justification explanations $(\beta=-.76, t=-1.52, p=.13)$, were also not significant, indicating that both control and explanations failed to moderate the relationship between privacy concerns and fairness perceptions. Therefore, Hypotheses 2 and 3 were not supported.

Hypothesis 4 proposed that applicant reactions in the form of overall fairness perceptions would predict distal applicant reactions outcomes such that more positive overall perceptions of fairness would relate to (a) decreased intentions to litigate, (b) increased purchasing intentions, (c) increased job acceptance intentions, (d) increased willingness to recommend employment opportunities with the company to others (recommendation intentions), and (e) higher levels of test taking motivation. Following the results of the Confirmatory Factor Analysis established three higher-order distal outcome variables (Litigation Intentions [H4a], Organizational Intentions [H4b], and Test-Taking Motivation [H4c]) and H4 was tested accordingly. First, correlation analysis was used to determine whether or not fairness perceptions were significantly related to litigation intentions, organizational intentions, and test-taking motivation. Then, hierarchical regression equation where the study control variables and study conditions were entered on the first step of the regression equation, and overall fairness perceptions were entered on the second step of the regression equation, was used to determine if overall fairness perceptions predicted each of the three distal outcomes. The overall models predicting litigation intentions $F(6,280)=3.62, p<.01$, organizational intentions $F(6,272)=5.43, p<.01$, test-taking motivation $F(6,279)=8.04, p<.01$ were 
significant. The regression equations are presented in Tables $10(\mathrm{H} 4 \mathrm{a}), 11(\mathrm{H} 4 \mathrm{~b})$, and 12 $(\mathrm{H} 4 \mathrm{c})$.

Results of the correlation analysis indicated that overall fairness perceptions were significantly related to litigation intentions $(r=-.15, p<.05)$, organizational intentions $(r$ $=.29, p<.01)$, and test-taking motivation $(r=.36, p<.01)$, lending support to Hypothesis 4a-c at the correlational level. Results from the hierarchical regression analysis indicated that higher overall perceptions of fairness predicted lower litigation intentions in the final equation $F(6,280)=3.62, p<.01$ with a significant $R^{2}$ change $=.02$, $\mathrm{p}<.05$, in support of Hypothesis $4 \mathrm{a}$. The hierarchical regression analysis indicated that higher overall perceptions of fairness predicted higher organizational intentions $F(6,272)$ $=5.43, p<.01$ with a significant $R^{2}$ change $=.08, p<.01$, in support of Hypothesis $4 \mathrm{~b}$. Finally, the hierarchical regression analysis indicated that higher overall perceptions of fairness predicted higher test-taking motivation $F(6,279)=8.04, p<.01$ with a significant $R^{2}$ change $=.11, p<.01$, in support of Hypothesis $4 \mathrm{c}$. Given results of the correlation analysis and subsequent hierarchical regression analyses, Hypothesis 4a, 4b, and $4 \mathrm{c}$ were supported.

\section{Tests for Mediation}

Hypothesis 5 proposed that applicant reactions in the form of overall fairness perceptions would mediate the relationship between privacy concerns and each distal applicant reactions outcome such that more positive overall perceptions of fairness would relate to (a) decreased intentions to litigate, (b) increased purchasing intentions, (c) increased job acceptance intentions, (d) increased willingness to recommend employment 
opportunities with the company to others (recommendation intentions), and (e) higher levels of test taking motivation. Confirmatory Factor Analysis previously established three higher-order distal outcome variables (Litigation Intentions [H5a], Organizational Intentions [H5b], and Test-Taking Motivation [H5c]) and H5 was tested accordingly. The results for the mediation hypotheses (Hypotheses 5a-c) can be found in Tables 12 (H5a), 13 (H5b), and 14 (H5c). Both Sobel Tests (Preacher \& Hayes, 2004; Sobel, 1982) and Bootstrapping (Preacher \& Hayes, 2008) were used to test overall perceptions of fairness in the proposed model. Consistent with McKinnon, Warsi, and Dwyer (1995), the Sobel Test z-value was obtained via the following equation where the critical values of the z-test (+/- 1.96) indicated whether or not mediation existed:

$$
z \text {-value }=(a * b) / \sqrt{ }\left(b_{2} * s a_{2}+a_{2} * s b_{2}\right)
$$

Bootstrapping, a more modern approach to testing mediation (Preacher \& Hayes, 2008), was used to confirm the Sobel Tests. The Bootstrapping procedures used a 95\% confidence interval and a total of 5000 samplings.

First, Hypothesis 5a was tested. As presented in Table 13, the final regression equation was consistent with tests of Hypothesis 1, and indicated that higher privacy concerns were associated with lower fairness perceptions $(\beta=-.18, t=-2.43, p<.05)$. Also, consistent with tests of Hypothesis 4, lower fairness perceptions were related to higher litigation intentions $(\beta=-.18, t=-2.15, p<.05)$. Last, privacy concerns had a negative, but non-significant effect on litigation intentions $(\beta=-.01, t=-.05, p=.96)$, suggesting that mediation was not possible. A Sobel test for mediation demonstrated that the indirect effect was non-significant $(z=1.61, p=.11)$. The Bootstrapping procedure 
yielded an indirect effect containing zero $(-.003, .10)$, thereby failing to provide support for Hypothesis $5 \mathrm{a}$.

Next, Hypothesis $5 \mathrm{~b}$ was tested. As presented in Table 14, the final regression equation was consistent with tests of Hypothesis 1, where higher privacy concerns were associated with lower fairness perceptions $(\beta=-.18, t=-2.31, p<.05)$. Consistent with tests of Hypothesis 4, higher fairness perceptions were associated with higher organizational intentions $(\beta=.36, t=5.36, p<.01)$. Finally, privacy concerns were associated with a significant positive effect on organizational intentions $(\beta=.19, t=2.21$, $p<.05)$. A Sobel test for mediation demonstrated that the indirect effect was significant $(z=2.12, \mathrm{SE}=.03, p<.05)$. The Bootstrapping procedure yielded an indirect effect that did not contain zero $(-.14,-.01)$, thereby demonstrating that fairness perceptions partially mediated the relationship between privacy concerns and organizational intentions in support for Hypothesis $5 \mathrm{~b}$.

Lastly, Hypothesis 5c was tested. As presented in Table 15, the final regression equation was consistent with tests of Hypothesis 1, where privacy concerns were negatively related to fairness perceptions in the final regression equation $(\beta=-.18, t=-$ $2.43, p<.05)$. Consistent with tests of Hypothesis 4 , fairness perceptions were positively related to test-taking motivation $(\beta=.45, t=6.30, p<.01)$. Finally, privacy concerns had a significant positive effect on test-taking motivation $(\beta=.18, t=1.97, p=.05)$. A Sobel test for mediation demonstrated that the indirect effect was significant $(z=2.27, \mathrm{SE}=$ $.04, p<.05)$. The Bootstrapping procedure yielded an indirect effect that did not contain zero $(-.16,-.01)$, thereby demonstrating that fairness perceptions partially mediated the 
relationship between privacy concerns and test-taking motivation in support for Hypothesis 5c.

\section{Research Questions}

Several research questions were posed as a part of this dissertation study. Research Question 1 sought to determine if the dimensions of privacy concerns, as measured by the CFIP, predicted fairness perceptions such that participants with (a) greater concerns over data collection, (b) greater concern over improper access, (c) greater concern over errors, and (d) greater concern over unauthorized secondary use experienced more negative overall perceptions of fairness. First, a CFA was conducted in order to determine if the CFIP was comprised of four different factors, including data collection, improper access, errors, and unauthorized secondary use. As previously reported, the CFIP's factor structure fit the data well. Second, a correlation analysis was conducted in order to determine the strength of the relationships between each scale factor and overall fairness perceptions. Lastly, hierarchical regression analysis was conducted where control variables and study conditions were entered on the first step of the regression equation. Then, each of the four factors of the CFIP were entered on the second step of the regression equation predicting overall fairness perceptions (Table 9).

Results of the correlation analysis indicated that concerns over data collection were negatively related to overall fairness perceptions $(r=-.19, p<.01)$, concerns over errors were negatively related to fairness perceptions $(r=-.03, p=.57)$, concerns over improper access were negatively correlated with fairness perceptions $(r=-.11, p<.05)$, and concerns over unauthorized secondary were significantly and negatively related to 
lower fairness perceptions $(r=-.12, p<.05)$. The overall hierarchical regression model was significant $F(9,271)=2.70, p<.01$, but the regression coefficients in the final equation indicated that the only scale that significantly added to the prediction of fairness was the CFIP's collection factor $(\beta=-.18, t=-2.24, p<.05)$. Differences between the relationships found using correlation analysis and those using hierarchical regression analysis may have been due to multicollinearity. Collinearity diagnostics for four factors of privacy concerns in the hierarchical regression model had low Eigen Values (.01 - .03) and high Condition Indices $(16.61-34.63)$, indicating that there were problems with multicollinearity among the variables included in the hierarchical regression. As Farrar and Glauber (1967) asserted, collinearity makes it difficult to "distinguish the independent contribution to explained variance of an explanatory variable" (p. 93). Other common indicators of collinearity among variables, including correlations between each factor, were significant, although not extremely high $(.26<r<.61)$. Though collinearity may have affected the hierarchical regression, the effect was probably not very large given the correlations between variables. As such, it is likely that unique variance in fairness perceptions is actually predicted by each of the four CFIP factors.

Research Question 2 sought to determine if a belief in legal protection predicted overall privacy concerns such that participants who had stronger beliefs that the law protects them would have lower privacy concerns. In order to test this research question, a correlation analysis was conducted in order to determine the strength of the relationship between participants' beliefs in legal protection and privacy concerns. Then, hierarchical regression analysis was conducted where control variables and study conditions were 
entered on the first step of the regression equation. Then, belief in legal protection was entered on the second step of the regression equation predicting overall privacy concerns (Table 16).

Results from the correlation analysis indicated that belief in legal protection was positively but not significantly related to privacy concerns $(r=.06, p=.28)$. Furthermore, the overall model for the hierarchical regression was non-significant $F(6,278)=1.34, p=$ .24. Belief in legal protection did not predict significant variance beyond other predictors in the regression model, as demonstrated in the final equation $(\beta=.08, t=1.40, p=.16)$, indicating that belief in legal protection was not related to privacy concerns. Post Hoc Analyses

Although not originally proposed in the hypotheses presented in this study, the aforementioned results suggested that several direct relationships existed between privacy concerns and the distal outcome variables (i.e., litigation intentions, organizational intentions, test-taking motivation). Therefore, hierarchical regression was used to determine whether or not control and/or explanations moderated these main effects. In order to determine whether or not control and explanations had a moderating effect on the relationship between privacy concerns and litigation intentions, control variables (i.e., Dichotomous Ethnicity, Internet Experience) were entered into the first step of a hierarchical regression equation. Then, the dummy-coded study conditions (i.e., Control vs. No Control, Excuse Explanation vs. No Justification Explanation, PrivacyJustification Explanation vs. No Privacy-Justification Explanation,) were entered into the second step of the regression equation. Third, overall privacy concerns were entered into 
the regression equation. Finally, the interaction between the control condition and privacy concerns, as well as the interactions between the explanation conditions and privacy concerns, were entered into the fourth and final step of the regression equation. The overall model was significant $F(9,277)=1.92, p<.05$.

Results of the hierarchical regression for privacy concerns predicting litigation intentions indicated that overall privacy concerns was not a significant predictor of litigation intentions in the third regression equation $(\beta=.02, t=.26, p=.80)$, and the relationship remained non-significant in the fourth regression equation $(\beta=.12, t=.85, p$ $=.40$ ). The main effects of the study conditions and the interaction terms were also nonsignificant.

Next, in order to determine whether or not control and explanations had a moderating effect on the relationship between privacy concerns and organizational intentions, the two control variables were entered into the first step of a hierarchical regression equation. Then, the dummy-coded study conditions were entered into the second step of the regression equation. Third, overall privacy concerns were entered into the regression equation. Finally, the interaction terms were entered into the fourth and final step of the regression equation. The overall model was non-significant $F(9,270)=$ $1.44, p=.17$

Results of the hierarchical regression for privacy concerns predicting organizational intentions indicated that overall privacy concerns was not a significant predictor of organizational intentions in the third regression equation $(\beta=.09, t=1.43, p$ $=.15)$, and the relationship remained non-significant in the fourth regression equation $(\beta$ 
$=.239, t=1.64, p=.10)$. The main effects of the study conditions and the interaction terms were also non-significant.

Finally, in order to determine whether or not control and explanations had a moderating effect on the relationship between privacy concerns and test-taking motivation, the two control variables were entered into the first step of a hierarchical regression equation. Then, the dummy-coded study conditions were entered into the second step of the regression equation. Third, overall privacy concerns were entered into the regression equation. Finally, the interaction terms were entered into the fourth and final step of the regression equation. The overall model significant $F(9,276)=2.39, p<$ .05 .

Results of the hierarchical regression for privacy concerns predicting test-taking motivation indicated that overall privacy concerns was not a significant predictor of testtaking motivation in the third regression equation $(\beta=.06, t=.98, p=.33)$, and the relationship remained non-significant in the fourth regression equation $(\beta=.20, t=1.40$, $p=.16)$. There was a significant main effect of control on test-taking motivation $(\beta=.12$, $t=2.07, p<.05)$, with control positively related to higher test-taking motivation, in the third regression equation. The fourth regression equation yielded different results. The main effect of control on test-taking motivation was non-significant $(\beta=-.23, t=-.50, p$ $=.62)$, but the main effect of excuse explanations was significant $(\beta=1.50, t=2.68, p=$ $.01)$. The interaction between privacy concerns and excuse explanations in predicting test-taking motivation was also significant $(\beta=-1.44, t=-2.58, p<.05$, indicating that excuse explanations moderated the relationship between privacy concerns and test-taking 
motivation. Participants who had low privacy concerns and received an excuse explanation had higher test taking motivation than those who had low privacy concerns and received no explanation. In contrast, the explanation seemed to have little effect on the test-taking motivation those with high privacy concerns. A graph of this interaction is found in Figure 13.

\section{Summary}

A graphical representation of the hypotheses tested in this dissertation study can be found in Figure 6. Overall, results were consistent with most of the proposed hypotheses. First, support was presented for a negative relationship between privacy concerns and fairness perceptions. Second, there was a lack of support for the proposals that control and explanations moderated the relationship between privacy concerns and fairness perceptions. Third, fairness perceptions were negatively related to litigation intentions, positively related to organizational intentions, and positively related to testtaking motivation. Fourth, fairness perceptions partially mediated the relationship between privacy concerns and organizational intentions as well as partially mediated the relationship between privacy concerns and test-taking motivation. Fairness perceptions did not mediate the relationship between privacy concerns and litigation intentions. Lastly, questions about belief in legal protection and the structure of predictive values of scales used in this study were answered. In all, the model of applicant reactions proposed in this dissertation study was partially supported. 


\section{Chapter 9:}

\section{Discussion}

The present study went beyond previous studies of applicant reactions in several important ways. Building on the work of previous authors from multiple disciplines (Bauer et al., 2006; Harris et al., 2003; Stewart \& Segars, 2002; Thibaut \& Walker, 1975), this study used a high-fidelity laboratory design when testing a model of applicant reactions that incorporated important determinants of applicant perceptions. Specifically, this study examined the role of privacy concerns, control, and explanations and the effect that these variables had on both proximal and distal applicant reactions outcome variables. The study used a valid measure of privacy concerns in an Internet-based selection context. As such, several important contributions were made from the results of this study. A graphical representation of the outcomes of the hypothesis tests are presented in Figure 6.

\section{Hypothesis Tests}

Results of Hypothesis 1, which proposed that privacy concerns were negatively related to fairness perceptions, underscores the importance of privacy concerns. As demonstrated by support of Hypothesis 1, privacy concerns are an important antecedent to fairness perceptions when participants complete an Internet-based selection process. Given that several control variables were used in this study (i.e., ethnic background, Internet experience), it is important to note that privacy concerns contributes to prediction of unique variance in fairness perceptions, regardless of an applicants' experience or knowledge-level with the Internet. 
Support for Hypothesis 1 is also a substantial contribution because the world of hiring has changed since the first comprehensive models of applicant reactions (e.g., Gilliland 1993), with the increase in use of Internet-based selection tools. The link between privacy concerns and fairness perceptions established by the results in this study clearly demonstrate the importance of measuring this variable in Internet-based selection contexts in order to account for more variance in the prediction of both fairness perceptions and important [distal] outcomes that directly affect organizations. Previous research has demonstrated the existence of several antecedents to fairness perceptions in a selection context, including test-type (Kluger \& Rothstein, 1993) and performance expectations (Bennett, Ordonez, \& Gilliland, 2003). This study adds to a list of possible antecedents that affect such perceptions, which provides for a plethora of new hypotheses to be proposed in an effort to identify factors that increase fairness perceptions among job applicants.

Not only does support for Hypothesis 1 contribute to a more comprehensive view of applicant reactions in an Internet-based selection context, but it also establishes an important link between the CFIP measure and commonly studied outcomes like fairness perceptions, litigation intentions, organizational intentions, and test-taking motivation. Relatively few articles have explored privacy concerns in the selection context (i.e., Bauer et al., 2006; Harris et al., 2003). Those few authors who have explored privacy concerns, to-date, have lacked a rigorous measure of privacy concerns designed for use in an Internet-based context. Results show that the CFIP predicts important perceptions and behavioral intentions. 
Gilliland's (1993) original model of applicant reactions (Figure 1) was a substantial contribution to applicant reactions theory not only because it was among the first comprehensive models of applicants reactions ever proposed, but also because it identified specific antecedents to fairness perceptions as well as specific distal reactions that stemmed from such fairness perceptions. In the early 1990s, however, Internet-based selection processes were rare, if non-existent. As such, privacy concerns related to Internet-based testing were unlikely to be salient in the minds of applicants and researchers. With an increase in the use of these tools, as well as an increase in research on applicant reactions, Gilliland's original model continues to be updated to reflect trends in applicant selection. This study demonstrates that privacy concerns are an important, valid addition to the antecedents of applicant reactions proposed in Gilliland's model. A link between privacy concerns and overall fairness perceptions was established through this research, providing for an expansion in the antecedents in Gilliland's model. Whereas past antecedents to justice/fairness perceptions focused on factors such as Human Resources (HR) Personnel, HR policies, and the type of test given to applicants (e.g., personality, cognitive ability), this study shows that new antecedents are related to risks associated with the testing context, such as the privacy concerns that applicants experience while participating in an Internet-based selection process. Hypotheses 2 and 3 were not supported in this study, as the moderating variables used as manipulations in this study (Control vs. No Control, Excuse Explanations vs. Privacy Justification Explanations vs. No Explanations) did not significantly moderate the relationship between privacy concerns and fairness perceptions. Still, these findings may be due to 
the laboratory context. Previous studies, such as the Truxillo et al. (2009) meta-analysis, demonstrated that the effect of moderators on applicant reactions may be attenuated in the laboratory environment. Therefore, the power of such moderators should not be immediately discounted by the results of this study. Even marginal results could still have great impact on an organization that uses Internet-based selection tools. It is important to note that one advantage commonly cited for Internet-based selection tools is the ability to assess a large quantity of applicants for one or more positions. Thousands of applicants participate in these processes every day (Handler \& Hunt, 2003), which means that thousands of peoples' perceptions of organizations may be altered. As such, even if the results of control and explanations in this study are non-significant, it could be that in a large online recruiting system the effects would be significant. In this case, presenting such control (in the form of choice) and/or explanations could be helpful in improving applicant reactions.

It is also important to note that participants had the opportunity to complete the entire selection process and then choose whether or not to release personal information to the "hiring manager", as this was the "control" condition. In this context, the control condition provided an applicant with a choice. By providing participants with a choice, it may have caused participants to ponder the risks involved in an online application, thereby increasing the saliency of a possibly threatening situation - mitigating control's positive effects on privacy concerns. Historically, the concept of control has been study in the context of privacy concerns (e.g., Stone \& Stone, 1990) but not studied in conjunction with fairness perceptions. Though this study proposed that control moderated 
the relationship between privacy concerns and fairness perceptions, this statistical relationship needs further examination. It is possible that control directly affects privacy concerns in the Internet-based selection context. Further research should investigate this idea.

Results demonstrated support for Hypotheses 4a-c, which proposed that fairness perceptions were (a) negatively related to litigation intentions, (b) positively related to organizational intentions, and (c) positively related to test-taking motivation. Support for this set of hypotheses was not surprising given findings from previous studies. For example, Bauer et al.’s (2001) results provided support for a negative relationship between fairness perceptions and litigation intentions. Hausknecht et al.'s (2004) metaanalysis established that multiple studies found positive relationship between fairness perceptions and organizational intentions variables. Finally, Schmit and Ryan (1997) reported a positive relationship between fairness perceptions and test-taking motivation. Support for Hypothesis 4a-c was critical, however, because it reaffirmed the relationships between fairness perceptions and distal outcomes while extending the findings to the Internet-based selection context.

Support for Hypothesis 5b-c, which proposed fairness perceptions mediated the relationship between privacy concerns and (b) organizational intentions and (c) testtaking motivation, was another significant contribution that should be of interest to future researchers for several reasons. First, support for Hypotheses $5 \mathrm{~b}$ and $5 \mathrm{c}$ are consistent with the notion that fairness items cluster together and function as one, higher-order construct, contrary to Colquitt et al. (2001), and then subsequently function as a mediator 
for distal outcomes similar to the event-mediation approach to organizational justice (Ambrose et al., 2007). Therefore, an event (in this case, an Internet-based selection process and resulting heightened privacy concerns) triggers a reaction in the form of decreased fairness perceptions, which results in more negative organizational intentions and decreased test-taking motivation. Hence, both the cognitive reaction (fairness perceptions) and the behavioral-intent reactions (organizational intentions, test-taking motivation) have been supported for privacy concerns in this model.

Interestingly, fairness perceptions did not mediate the relationship between privacy concerns and litigation intentions as proposed in Hypothesis 5a. Perhaps to applicants, some other antecedent influences litigation intentions through the mediating variable of fairness perceptions. Therefore, it may be important to consider other variables related to litigation intentions in order to parse this relationship. For example, particular factors of the privacy concerns variable may be more likely to affect litigation intentions directly or through fairness perceptions. Future research could explore these variables, along with other variables such as whether or not the provision of information about participants' legal rights influences litigation intentions. Despite whether or not the relationships between privacy concerns and litigation intentions in this study were significant, it is important to note the large real-world effect that just one applicant can have on an organization through his/her litigation intentions and resulting litigation behavior. Just one legal claim by an applicant could cost an organization not only monetarily, but also in terms of the organization's reputation. 
Fairness perceptions mediated the relationship between privacy concerns and organizational intentions; however, Organizational Justice Theory may not be the only way to explain this finding. One explanation for this mediating relationship is rooted in Psychological Contract Theory (Argyris, 1960; Rousseau, 1989). Consistent with Psychological Contract Theory, an applicant may perceive an implicit, relational contract where applicants have trust that their privacy is protected by the hiring organization. The contract is then breached by an Internet-based selection procedure that is perceived as threatening. Though belief in legal protection was measured in this study, belief in the implicit contract between the organization and the applicant was not. Therefore, a future direction of research may involve the measurement of beliefs in a hiring organization's responsibility to applicants to safeguard them from harm.

Fairness perceptions mediated the relationship between privacy concerns and testtaking motivation. Increased privacy concerns may place individuals in an arousal state, increasing stress or anxiety. This additional arousal may result in reduced fairness perceptions and decreased test-taking motivation. Such arousal may provide a primacy effect during the selection process that affects fairness perceptions and thereby decreases levels of test-taking motivation. Consistent with Fairness Heuristic Theory (Lind et al., 2001), fairness perceptions remain low without a salient action by the organization. Providing personal information such as giving part of the social security number may act as a negative event from which it is difficult for the organization to recover, especially for applicants with high privacy concerns. This could negatively affect outcome variables including test-taking motivation as well as organizational intentions by placing applicants 
in a state where they feel more negative about the organization. In other words, applicants could feel as though the organization wants to eliminate them rather than select them, resulting in more negative organizational intentions and lower test-taking motivation. Unfortunately, the moderators proposed in this study did not appear to serve as "salient actions" that could change this negative perception, but future research should consider moderators that may positively alter the privacy concerns - fairness perceptions relationship in order subsequently increase participants' test-taking motivation and even organizational intentions. Future research should also consider a measure of test-taking anxiety or stress in relation to privacy concerns in order to more carefully study these relationships.

Post hoc analyses revealed several important findings relevant to the study of applicant reactions to privacy concerns. First, there was a main effect of control on testtaking motivation. Perhaps "control" situations place applicants in a psychological state where they feel as though they also have control over their test-performance. Second, the interaction between privacy concerns and excuse explanations demonstrates that explanations result in higher levels of test-taking motivation for both low and high privacy concerns applicants. This effect may also be due to a psychological state that applicants experience, as they are placed into a situation where they feel as though the best candidate will be selected rather than candidates eliminated, as the excuse explanation used in this study eludes. Finally, it may be that the excuse explanation only works for those in the with low privacy concerns because, for those who are very 
concerned with privacy, no explanation (or other intervention) can really alleviate such high concerns.

This study also presented findings related to research questions and control variables that are important to note. For example, Research Question 2 asked if a belief in legal protection would be related to privacy concerns. As found in this study, belief in legal protection was not related to privacy concerns. These results suggest that privacy concerns may not be viewed by applicants as a situation that can easily solved after-thefact. For example, in the case of identity-theft, damage may be done that is so difficult to repair that legal action is not seen as viable or worth the investment. Given that the notion of "legal protection" may be only important in situations where reparations can be made, applicants may not be affected by their beliefs in legal protection. Still, "belief in legal protection" is a very abstract concept and its measurement is difficult. The abstract nature of the "belief in legal protection" construct and the lack of a strong measure may explain the null results found here.

As demonstrated by analyses that identified control variables for use in this study, ethnic background differences existed in relation to litigation intentions. Individuals who were Non-Caucasian/Non-White had higher litigation intentions then Caucasian/White participants, although this difference was not large. It is possible that individuals belonging to ethnic background minority groups are more familiar with their rights due to legal status as a protected class. For that matter, some members of other protected classes, such a women, may be more likely to litigate. As seen in cases such as Dukes v. Wal-Mart Stores, Inc. (2010), women may also be more likely to litigate, indicating that 
there may be some groups that are more likely to pursue legal action than others when faced with processes perceived as unfair. This could be driven by not only actual events, but also historical trends in discrimination. It is important to note that many variables may intervene in this relationship, and simply belonging to a particular protected class may not make one more likely to litigate. Future research should look at litigation intentions among different protected classes to discern the cognitive reactions that lead to litigation intentions.

\section{Measures}

Confirmation of the structure of scales used in this study was important because it bolstered the integrity of the study findings by establishing the statistical rigor of the measures. Scales used to measure variables that were central to this study, such as "organizational intentions" and "concern for information privacy," were confirmed as meaningful measures of distinct psychological constructs through statistical analysis.

Distal outcomes, such as measures of behavioral intentions, are important to measure with accuracy because they are often the outcomes most meaningful to the practice of organizations. As demonstrated in this study, several distal outcome variables functioned similarly. For example, recommendation intentions, job acceptance intentions, and purchasing intentions functioned as one variable named in this study "Organizational Intentions" - therefore, one may conclude that behavioral intentions focused on an organization are closely related. It also suggests that in the minds of applicants there is a general underlying perception of the organization, with little differentiation among factors such of their intentions to purchase and the attractiveness of the organization. In 
fact, future researchers may choose to measure general perceptions of and intentions toward the organization or develop a scale for general organizational intentions in order to gain more insight into this area while using fewer scale items. Given the difficulty that study participants experience when responding to hundreds of items during the course of a rigorous study, this could prove helpful in helping to reduce error in the measure due to a lack of participant attention.

However, in the measurement realm, perhaps the greatest contribution of the present study come from the primary variable of interest in this study, privacy concerns, which was measured via the Concern for Information Privacy Scale (CFIP). The CFIP appeared to be a valuable, statistically-sound way of measuring privacy concerns in the Internet-based selection process. Although the CFIP was not developed for the applicant reactions context, it held its previously identified structure within the Internet-based selection context, demonstrating that the minor adjustments made to the scale in order to fit this context were successful. Internal consistency for the scale was high $(\alpha=.91)$, and the four-factor structure - which included the factors of collection, improper access, errors, and unauthorized secondary use- established by previous authors (Stewart \& Segars, 2002) was supported via Confirmatory Factor Analysis in the present study. Moreover, although all four of these dimensions were found to relate to applicant reactions, only collection was found to predict unique variance in the regression equations.

Interestingly, as found through CFA and a test of Research Question 1, the CFIP is comprised of four factors, including collection, improper access, errors, and 
unauthorized secondary use. Still, only one factor (collection) uniquely predicted fairness perceptions. Collection items such as (1) "It usually bothers me when Web sites ask me for personal information," (2) "When Web sites ask me for personal information, I sometimes think twice before providing it," (3) "It bothers me to give personal information to so many Web sites," and (4) "I'm concerned that Web sites are collecting too much personal information about me" expose the cognitive nature of privacy concerns, with participants' responses prompted by words such as "bothers" and "concerned." The other three established factors of the CFIP (improper access, errors, and unauthorized secondary use) focus more on organizational responsibility and what participants believe organizations should do. The results suggest that reducing privacy concerns and subsequently increasing fairness perceptions among job applicants may be partially influenced by factors outside of the control of an organization, as these concerns may be driven by general perceptions about Internet protection rather than perceptions of a specific Internet-based selection system or organization. Future studies may want to further refine the measure. Perhaps the collection factor drives the overall CFIP score such that other items could be eliminated.

Another unique finding involved the examination of the pre-test and post-test distal outcome variable means across study conditions. As outlined in the study procedure, litigation intentions, organizational intentions (i.e., job acceptance intentions, purchasing intentions, recommendation intentions), and test-taking motivation were measured both before and after individuals participated in the fictitious Internet-based selection process. As shown in Table 17, an examination of scale means across study 
conditions indicated that litigation intentions increased pre-test to post-test measurement timing. Conversely, organizational intentions and test-taking motivation decreased from pre-test to post-test measurement timing in every condition. Therefore, individuals' reactions were more negative following participation in the fictitious selection procedure. Taken at face-value, this finding may indicate that participation in an Internet-based selection process may result in negative reactions. Still, more research is needed to determine the reason for this effect. For example, this effect may be an artifact of the laboratory context, as participant reactions could be more salient to participants after participation in the fictitious process because the context becomes more "real" to individuals.

\section{Theoretical Implications}

This dissertation study approached the investigation of applicant reactions in the Internet-based selection context from an organizational justice approach while incorporating findings from the information sciences field. As such, several important implications for theory can be derived from the study results.

First, this study lends further support to Ambrose et al.'s (2007) findings that organizational justice variables (i.e., distributive justice, informational justice, interpersonal justice, and procedural justice) similarly predict overall attitudes toward the organization. The structure of hypotheses tested in this model involves reactions to an event (privacy concerns stemming from the Internet-based selection process) triggering a cognitive response (fairness perceptions) which results in behavioral intentions (organizational intentions). Thus, this study lends credence to the idea that organizational 
justice variables may function as a single construct, as proposed in the monistic theory of organizational justice (Cropanzano \& Ambrose, 2001). Still, it is worthy to note that the mediating effect of fairness perceptions may be an effect unique to the context of applicant selection, as selection contexts present salient opportunities for individuals to obtain a position with an organization that would provide financial security for obtaining and maintaining the most basic resources (such as food and shelter). For this reason, selection contexts have been referred to as "high stakes" by researchers such as Sackett, Schmitt, Ellingson, and Kabin (2001, p. 302). Research that compares the function of justice variables across different contexts may be helpful in further specifying monistic versus four-factor conceptualizations of justice.

Second, this dissertation study demonstrates the benefit to an interdisciplinary approach to research in the field of industrial/organizational psychology. Though interdisciplinary approaches may require additional time and energy due to a lack of familiarity with the literature in other fields (Thagard, 2010), valuable contributions from literature outside of the field exist. For example, the contribution of the Concern for Information Privacy scale to this study, which originates from the information sciences field, provided both a prima facie and statistically sound way to conceptualize applicant privacy concerns. Researchers should continue to look beyond the boundaries of their own fields, as there are valuable findings originating from many different theoretical orientations that can be applied to pioneering research. 
Future Research

Several proposals for future research are suggested from the results of the present dissertation study. Such proposals include calls for research on variables not studied in this dissertation, the study of beliefs about organizations' responsibilities, the study of anxiety and arousal in the Internet-based selection context, the study of ethnic background variables in relation to Internet-based selection processes, and further study of the CFIP measure. In order to investigate these proposals further, it is necessary to contemplate how future research on this topic area should be designed.

First, future research should seek to conduct a similar study with not only a larger participant sample, but also a sample of participants who are applying for jobs in a realworld context. This would assist with the engagement level of participants such that they would be more aware of the study conditions and would provide the opportunity to compare the reactions of participants from varying ethnic backgrounds. Furthermore, real world applicants could receive pre-test privacy concern measures weeks before participation in order to fully separate the antecedent (privacy concerns) and mediator (fairness perceptions). Participants could also receive follow-up notifications of rejection that provide explanations several days after the post-test, rather than immediately following participation, as in the present study. This may not only be more realistic to applicants, but would allow for follow-on surveys to measure long-term litigation intentions, organizational intentions, and test-taking motivation. Such measurements could be compared over time, which may help to determine if variables such as job scarcity affect privacy concerns and applicant reactions. Two-way interactions (e.g., 
interactions between control and explanations) as well as three-way interactions (e.g., interactions between privacy concerns, control, and explanations) could also be further investigated, hypothesized, and tested in supporting analyses when conducting future studies.

Other study designs should also consider alternative measures and new variables of interest. For example, future studies could include measures of privacy concerns that are less obvious, so as to reduce the chances that participants are primed. Such measures could include word association tests or may even involve measuring the amount of time individuals spend reading privacy notifications. Related to the measurement of privacy, future studies may explore whether or not fairness mediates relationships between each of the 4 dimensions of privacy (as measured by the CFIP) and the distal outcome variables. In terms of studies that may measure other concepts related to privacy concerns and Internet-based selection, other studies could explore the type of data collected from applicants and how the different types of data (e.g., cognitive ability, contact information, social security number) related to the different dimensions of applicant privacy concerns explored in this study. Future researchers may also be interested in individuals' willingness to participate in Internet-based procedures, as it may be related to individual difference variables such as openness to experience (i.e., personality variables). Finally, other research could be designed to measure whether or not providing some types of information about privacy protection, as is common on many Internet shopping sites, could actually frighten participants, as some descriptions may unveil issues that applicants may have never considered. 
Second, future research could eliminate items that measure belief in legal protection and further refine measures of concern for information privacy (the CFIP scale), organizational intentions, and fairness perceptions. For example, several items were used to measure fairness in this study that were not used during analysis, including a short-form of the Selection Procedural Justice Scale (SPJS). With the elimination of these items, additional measures could be used in future research to measure test-taking anxiety, beliefs about organizational responsibilities, and actual behavior without significantly lengthening the process for participants.

Finally, future research should seek to measure actual behavior of applicants. If a similar study is performed in a real-world context, participants could be asked to provide information about the purchase of goods from the organization, the number of people to whom they recommended the organization for employment, and the actual acceptance of job offers. Legal action against the organization could also be tracked with a large sample size.

\section{Implications for Practice}

The findings of this study indicate that privacy concerns are important; they have the ability to affect applicants' behavioral intentions toward the organization through fairness perceptions, including their intentions to recommend the organization to other applicants, their intentions to accept a job offer, and their intentions to make purchases from the organization. Furthermore, applicants' motivation to perform well on the selection test is affected through fairness perceptions, which could result in otherwise acceptable applicants performing at unacceptable levels, thereby restricting the talent 
available to organizations. Finally, privacy concerns may be directly related to applicants' intentions to make a legal claim against the organization, which could directly affect organizations' reputations and financial-standings.

Given the aforementioned consequences of privacy concerns, organizations should consider ways to reduce privacy concerns among applicants. Two strategies were explored in this study, including providing more control to applicants and providing applicants with explanations for the use of the Internet-based selection test. Though these manipulations did not significantly moderate the relationship between privacy concerns and fairness perceptions as proposed in this study, they may still be good practices. Given that previous research has shown the power of explanations in other selection contexts (Truxillo et al., 2009), this strategy is easy to implement, cost-effective, and worthwhile for organizations to implement. Furthermore, providing applicants with control was helpful at increasing fairness perceptions, although not statistically significant in this laboratory context. Excuse explanations and privacy concerns also appeared to interact to affect test-taking motivation, indicating that explanations could be helpful, in some way, with motivating applicants to do well when they have lower privacy concerns. Organizations should also consider interventions not explored in this study. For example, tests that are highly face valid may be preferable, as a personality-based test was used in a simulated Internet-based selection process designed for this study. Content of the Internet-based selection test (willingness to be drug tested, willingness to have a background check) may also have affected privacy concerns. Organizations may wish to use caution when using these types of measures in an Internet-based selection context, as 
they may be seen as more harmful should results be inadvertently released to a thirdparty. Questions like these could be asked in an interview rather than via Internet-based procedures.

Though the "collection" factor associated with the measure of privacy concerns predicted unique variance in fairness perceptions, it is important to note that participants in this study rated "unauthorized secondary use" as a higher concern than the other three privacy concerns factors $(M=4.62)$. Though not proposed in this study, this factor may be a direct predictor of litigation intentions. Organizations may want to make salient the specific safeguards they have utilized in order to reduce the chances that third-parties would acquire and use data from applicants. Furthermore, providing applicants with "control" may have altered the relationship between privacy concerns regarding collection and overall fairness perceptions. Though this specific statistical relationship was not hypothesized, it could be a future focus of research.

\section{Potential Limitations}

Though much thought and care were used to design a high-fidelity laboratory study, several limitations existed. Such limitations included the study context, participants' attributes, restriction-of-range among study participants, and the variables manipulated.

The laboratory context provided greater control over variables but did not provide the most realistic context for participants, as would a real-world study. As demonstrated in previous meta-analytic research (i.e., Truxillo et al., 2009), effect sizes for laboratory studies in applicant reactions are lower than that of real-world studies, suggesting that 
any effects found in this study may be an underestimate. The proposed study sought to create the most realistic test-taking environment possible through the use of an Internetbased selection test that closely resembles those that are currently used by employers. In addition, the laboratory environment provided for greater control over manipulations, as well as the measurement of variables that were more difficult and therefore rarely measured in real-world applicant selection contexts, such as litigation intentions. Though a $\$ 100$ prize was given to the "participant who was hired for the job," this prize may not have been enough to provide motivation for laboratory participants to do well on the test, and they may not have cared about the risks associated with Internet-based selection procedure in the same way as a real-world applicant. Still, the laboratory setting is probably the best setting for such a study, as it would be difficult to perform these manipulations among an actual sample of applicants, assigning them to different conditions, for legal and ethical reasons. Furthermore, a true experimental design with random assignment allows researchers to test causal hypotheses (Chambliss \& Schutt, 2006), as performed in this study.

The nature of students applying for a hypothetical job may limit the generalizability of the study results. Student participants provide a restricted-range of education, age, and socio-economic status. Also, all participants were currently enrolled in college, inferring a restriction in range in socio-economic status, though this was not measured in this study. However, the average age of the participants (26) was higher than in many other student sample studies and approximately one-third of the participants were "currently seeking" jobs. Thus, the students were generally older than most other 
student samples and the topic of the study was one with which many had experience, providing greater generalizability to real-world contexts than similar studies with student participants.

Further restriction-of-range of study participants, resulting in withdrawal bias, may be a limiting factor to generalizability of the study results. Given that the main variables of the study involve privacy concerns and the Internet, the participants with the highest concern may have selected to do the alternate assignment or have dropped-out of the study before completion. In fact, participants included in the final dataset had a very narrow range of privacy concerns, as demonstrated by a small standard deviation $(\mathrm{SD}=$ .55). This may indicate little variability in privacy concerns and may have influenced the ability to identify differences between participants who received different manipulations. Furthermore, based on the raw number of participants who chose to complete an alternate assignment as well as the number of participants who only completed a portion of the study procedure, it is estimated that approximately 100 participants chose not to participate in the study after viewing the informed consent. Those individuals with the highest of privacy concerns may have dropped-out because they were not only required to use the Internet, but the study also required them to disclose personal information via this mode. However, there were no substantial differences between this group and the other students who remained in the sample, thus reducing concerns about non-response bias.

The moderators explored in this study may not have been salient to the participants. Given the long duration of the study (approximately 30 minutes), in which 
participants had to respond to over 170 items, it is possible that participants may not have noticed the control manipulation or the explanation manipulation(s). Future studies may wish to explore these two variables without measuring the distal outcomes measured in this study in order to reduce the number of items and focus on the two manipulations. Additionally, common method bias may have been present in this study, as participants' concerns about privacy while participating in the Internet-based selection process could have been affected by the manipulations themselves. For example, some participants may not have assumed that there was any risk inherent in submitting private information over the Internet. However, the privacy justification explanation condition could have raised questions in the mind of such participants by prompting them to consider this reality. However, I attempted to address this issue by placing the privacy concerns variable after the selection process, based on my pilot results. This may have mitigated some of these issues with privacy concerns.

Finally, the issue of measuring antecedents, mediators, and outcomes concurrently presents an opportunity for improvement of study design. Ideally, variables such as privacy concerns, fairness perceptions, and the distal outcomes of litigation intentions, organizational intentions, and test-taking motivation would be separated by time in order to most realistically measure behavioral intentions. Still, for reasons of practicality, this study used concurrent measurement, although participants first received the CFIP scale, then the fairness scales, and lastly the distal outcome scales. Future research should seek to incorporate longitudinal measures. 


\section{Conclusion}

This dissertation study tested a model of applicant reactions in the Internet-based selection context, which is a growing context for job applications. The results of the study provided a more comprehensive view of the issues that affect applicant reactions. They thus expand on past models of applicant reactions that were developed prior to Internet testing and make it clear that applicants' privacy concerns are important. From this research, one may conclude that privacy concerns are an important predictor of fairness perceptions as well as distal outcomes important to organizations. Future research should seek to identify moderators that increase fairness perceptions among applicants in realworld contexts. Such research will not only provide a more comprehensive view of applicant reactions, but could also seek to help both applicants and organizations by increasing test-taking motivation, decreasing litigation intentions, and increasing positive organizational intentions. 
Table 1. Table of Experimental Conditions

\begin{tabular}{|c|l|l|}
\hline & \multicolumn{1}{|c|}{ Control Condition } & \multicolumn{1}{c|}{ Explanation Condition } \\
\hline 1 & Control & Excuse Explanation \\
\hline 2 & Control & Privacy Justification Explanation \\
\hline 3 & Control & No Explanation \\
\hline 4 & No Control & Excuse Explanation \\
\hline 5 & No Control & Privacy Justification Explanation \\
\hline 6 & No Control & No Explanation \\
\hline
\end{tabular}


Table 2. Listing of Measures

\begin{tabular}{|c|c|c|}
\hline Measure/Scale & Timing & $\begin{array}{l}\text { Number of } \\
\text { Items }\end{array}$ \\
\hline Concern for Information Privacy (CFIP) & $\begin{array}{l}\text { Pre-Test (Pilot } \\
\text { Study) }\end{array}$ & 15 \\
\hline $\begin{array}{l}\text { Test-Taking Motivation (Valence, Instrumentality, } \\
\text { Expectancy Motivation Scale) (VIEMS) }\end{array}$ & Pre-Test & 10 \\
\hline Belief in Legal Protection & Pre-Test & 3 \\
\hline Electronic App Preference & Pre-Test & 3 \\
\hline Electronic Application Preference & Pre-Test & 3 \\
\hline Recommendation Intentions & Pre-Test & 3 \\
\hline Job Acceptance Intentions & Pre-Test & 2 \\
\hline Purchasing Intentions & Pre-Test & 3 \\
\hline Litigation Intentions & Pre-Test & 4 \\
\hline Internet-Based Job Application & Test Procedure & 30 \\
\hline Personality Test & Test Procedure & 20 \\
\hline CFIP & Post-Test & 15 \\
\hline SPJS & Post-Test & 14 \\
\hline Procedural Justice & Post-Test & 7 \\
\hline Distributive Justice & Post-Test & 4 \\
\hline VIEMS & Post-Test & 10 \\
\hline Belief in Legal Protection & Post-Test & 3 \\
\hline Electronic Application Preference & Post-Test & 3 \\
\hline Recommendation Intentions & Post-Test & 3 \\
\hline Job Acceptance Intentions & Post-Test & 2 \\
\hline Purchasing Intentions & Post-Test & 3 \\
\hline Litigation Intentions & Post-Test & 4 \\
\hline Perceived Knowledge and Experience with the Internet & Post-Test & 2 \\
\hline Employment Status & Post-Test & 3 \\
\hline
\end{tabular}


Table 2. Listing of Measures (Continued)

\begin{tabular}{|l|l|c|}
\hline \multicolumn{1}{|c|}{ Measure/Scale } & \multicolumn{1}{c|}{ Timing } & $\begin{array}{c}\text { Number of } \\
\text { Items }\end{array}$ \\
\hline Facebook Privacy & Post-Test & 2 \\
\hline Demographics & Post-Test & 2 \\
\hline Manipulation Check & Post-Test & 3 \\
\hline Total Number of Scale Measures & & 126 \\
\hline Total Number of Test Procedure Measures & & 50 \\
\hline Grand Total of All Measures & & 176 \\
\hline & & \\
\hline
\end{tabular}


Table 3. Means, Standard Deviations, Correlations, and Internal Consistency Estimates for Pilot Study Variables

\begin{tabular}{|c|c|c|c|c|c|c|c|c|c|c|c|}
\hline & Variable & M & $\mathrm{SD}$ & 1 & 2 & 3 & 4 & 5 & 6 & 7 & 8 \\
\hline 1 & Age & 26.06 & 7.96 & -- & & & & & & & \\
\hline 2 & Internet Experience & 7.56 & 1.62 & .07 & -- & & & & & & \\
\hline 3 & Internet Knowledge & 7.18 & 1.70 & -.01 & $.75 * *$ & -- & & & & & \\
\hline 4 & CFIP (Pre-Test) & 4.22 & 0.35 & $-.43 * *$ & -.06 & .10 & $(.79)$ & & & & \\
\hline 5 & CFIP (Post-Test) & 4.19 & 0.61 & $-.40 * *$ & -.07 & .07 & $.74 * *$ & $(.92)$ & & & \\
\hline 6 & Procedural Justice & 2.53 & 0.86 & -.07 & .24 & .15 & -.02 & .06 & $(.91)$ & & \\
\hline 7 & Distributive Justice & 2.39 & 1.02 & -.03 & .10 & .08 & .31 & .16 & $.73 * *$ & $(.91)$ & \\
\hline 8 & SPJS & 2.42 & 0.77 & -.22 & .00 & -.04 & .16 & .10 & $.84 * *$ & $.72 * *$ & $(.92)$ \\
\hline 9 & VIEMS (Pre-Test) & 4.00 & 0.77 & $.56^{* *}$ & .22 & .13 & $.48 *$ & .10 & .06 & .03 & -.03 \\
\hline 10 & VIEMS (Post-Test) & 3.71 & 0.77 & -.18 & .31 & .23 & .18 & .16 & $.39^{*}$ & .22 & $.41^{*}$ \\
\hline 11 & Litigation Intentions (Pre-Test) & 2.49 & 0.93 & $-.34 *$ & $-.49 * *$ & -.21 & .26 & .22 & .14 & .28 & $.33^{*}$ \\
\hline 12 & Litigation Intentions (Post-Test) & 2.64 & 1.05 & .00 & .04 & .12 & .40 & .12 & .30 & $.49 * *$ & .27 \\
\hline 13 & Job Acceptance Intentions (Pre-Test) & 4.18 & 0.99 & .13 & .26 & .12 & .10 & .13 & -.08 & $-.35 *$ & -.24 \\
\hline 14 & Job Acceptance Intentions (Post-Test) & 3.88 & 1.08 & .28 & .19 & .13 & -.01 & .09 & .23 & -.08 & .08 \\
\hline
\end{tabular}


Table 3. Means, Standard Deviations, Correlations, and Internal Consistency Estimates for Pilot Study Variables (Continued)

\begin{tabular}{|c|c|c|c|c|c|c|c|c|c|c|c|}
\hline & Variable & $\mathrm{M}$ & SD & 1 & 2 & 3 & 4 & 5 & 6 & 7 & 8 \\
\hline 15 & Recommendation Intentions (Pre-Test) & 3.36 & 0.94 & -.04 & .29 & .05 & .11 & .24 & .13 & -.10 & .06 \\
\hline 16 & Recommenation Intentions (Post-Test) & 3.09 & 1.10 & .12 & $.33 *$ & .15 & .07 & .08 & $.47 * *$ & .26 & $.38 *$ \\
\hline 17 & Purchase Intentions (Pre-Test) & 3.68 & 0.72 & .14 & .05 & -.09 & .23 & .27 & -.09 & -.23 & -.16 \\
\hline 18 & Purchase Intentions (Post-Test) & 3.43 & 0.79 & .22 & .02 & -.13 & .09 & .13 & $.34^{*}$ & .21 & $.32 *$ \\
\hline 19 & Belief in Legal Protection (Pre-Test) & 3.38 & 1.00 & -.03 & .19 & .05 & .06 & .02 & .03 & -.18 & -.07 \\
\hline 20 & Belief in Legal Protection (Post-Test) & 3.22 & 1.06 & .02 & .18 & -.04 & -.12 & -.12 & -.01 & -.16 & -.07 \\
\hline 21 & Electronic Application Preference & 3.06 & 1.33 & .16 & .23 & .30 & .14 & .04 & .14 & -.09 & .11 \\
\hline
\end{tabular}


Table 3. Means, Standard Deviations, Correlations, and Internal Consistency Estimates for Pilot Study Variables (Continued)

\begin{tabular}{|c|c|c|c|c|c|c|c|}
\hline & Variable & 9 & 10 & 11 & 12 & 13 & 14 \\
\hline 10 & VIEMS (Post-Test) & .18 & $(.91)$ & & & & \\
\hline 11 & Litigation Intentions (Pre-Test) & $-.33 *$ & -.05 & $(.95)$ & & & \\
\hline 12 & Litigation Intentions (Post-Test) & .15 & .13 & $.51 * *$ & $(.97)$ & & \\
\hline 13 & Job Acceptance Intentions (Pre-Test) & $.55^{* *}$ & .29 & $-.33 *$ & -.16 & -- & \\
\hline 14 & Job Acceptance Intentions (Post-Test) & $.52 * *$ & $.37 *$ & -.22 & -.06 & $.69 * *$ & -- \\
\hline
\end{tabular}


Table 3. Means, Standard Deviations, Correlations, and Internal Consistency Estimates for Pilot Study Variables (Continued)

\begin{tabular}{|c|c|c|c|c|c|c|c|c|c|c|c|c|c|c|}
\hline & Variable & 9 & 10 & 11 & 12 & 13 & 14 & 15 & 16 & 17 & 18 & 19 & 20 & 21 \\
\hline 15 & $\begin{array}{l}\text { Recommendation Intentions (Pre- } \\
\text { Test) }\end{array}$ & $.45^{* *}$ & $.46^{* *}$ & -.27 & -.02 & $.66^{* *}$ & $.48 * *$ & $(.87)$ & & & & & & \\
\hline 16 & $\begin{array}{l}\text { Recommendation Intentions } \\
\text { (Post-Test) }\end{array}$ & $.35 *$ & $.59 * *$ & -.28 & .03 & $.38 *$ & $.65^{* *}$ & $.61 * *$ & $(.92)$ & & & & & \\
\hline 17 & Purchase Intentions (Pre-Test) & .56 & -.10 & -.07 & -.05 & $.59 * *$ & $.39 *$ & $.41^{*}$ & .06 & $(.77)$ & & & & \\
\hline 18 & Purchase Intentions (Post-Test) & $.37 *$ & .29 & -.01 & .07 & $.33 *$ & $.68 * *$ & .24 & $.59 * *$ & $.39 *$ & $(.80)$ & & & \\
\hline 19 & $\begin{array}{l}\text { Belief in Legal Protection (Pre- } \\
\text { Test) }\end{array}$ & $.45^{* *}$ & .32 & -.10 & .06 & $.59 * *$ & $.51 * *$ & $.60 * *$ & .30 & $.39 * *$ & $.37 *$ & $(.92)$ & & \\
\hline 20 & $\begin{array}{l}\text { Belief in Legal Protection (Post- } \\
\text { Test) }\end{array}$ & .32 & .31 & -.13 & .08 & $.45^{* *}$ & $.48 * *$ & $.42 * *$ & .31 & .30 & $.44 * *$ & $.89 * *$ & $(.93)$ & \\
\hline 21 & $\begin{array}{l}\text { Electronic Application } \\
\text { Preference }\end{array}$ & .20 & .30 & -.02 & .05 & .14 & $.48 * *$ & .13 & $0.38^{*}$ & -.02 & $.46^{* *}$ & $.33^{*}$ & $.33^{*}$ & $(.96)$ \\
\hline
\end{tabular}

Note. ${ }^{* *} p<.001 .{ }^{*} p<.05 . n=26-48$. Coefficient alphas found on diagonal. Measures 4 - 21 are on a 5-point scale. Measures 2 - 3 are on a 10 point scale.

VIEMS $=$ Valence, Instrumentality, Expectancy Motivation Scale.

CFIP $=$ Concern for Information Privacy Scale. SPJS = Selection Procedural Justice Scale. 
Table 4. Summary of ANOVA: Differences between Central Florida Sample and Pacific Northwest Sample

\begin{tabular}{|c|c|c|c|c|c|}
\hline Post-Test Measure & & $\begin{array}{l}\text { Sum of } \\
\text { Squares }\end{array}$ & $\mathrm{df}$ & $\begin{array}{l}\text { Mean } \\
\text { Square }\end{array}$ & $F$ \\
\hline \multirow[t]{3}{*}{ Test-Taking Motivation } & Between Groups & 15.40 & 1 & 15.40 & $18.86^{* *}$ \\
\hline & Within Groups & 302.92 & 371 & .82 & \\
\hline & Total & 318.32 & 372 & & \\
\hline \multirow[t]{3}{*}{$\begin{array}{l}\text { Recommendation } \\
\text { Intentions }\end{array}$} & Between Groups & 25.02 & 1 & 25.02 & $22.22 * *$ \\
\hline & Within Groups & 422.20 & 375 & 1.13 & \\
\hline & Total & 447.22 & 376 & & \\
\hline \multirow[t]{3}{*}{ Job Acceptance Intentions } & Between Groups & .56 & 1 & .56 & .66 \\
\hline & Within Groups & 311.33 & 369 & .84 & \\
\hline & Total & 311.89 & 370 & & \\
\hline \multirow[t]{3}{*}{ Purchasing Intentions } & Between Groups & 13.50 & 1 & 13.50 & $18.72 * *$ \\
\hline & Within Groups & 270.40 & 375 & .72 & \\
\hline & Total & 283.90 & 376 & & \\
\hline \multirow[t]{3}{*}{ Litigation Intentions } & Between Groups & 2.38 & 1 & 2.38 & 2.29 \\
\hline & Within Groups & 390.24 & 376 & 1.04 & \\
\hline & Total & 392.61 & 377 & & \\
\hline \multirow[t]{3}{*}{ Procedural Justice } & Between Groups & 46.72 & 1 & 46.72 & $73.36^{* *}$ \\
\hline & Within Groups & 234.37 & 368 & .64 & \\
\hline & Total & 281.09 & 369 & & \\
\hline \multirow[t]{3}{*}{ Distributive Justice } & Between Groups & 66.60 & 1 & 66.60 & $69.57 * *$ \\
\hline & Within Groups & 360.93 & 377 & .96 & \\
\hline & Total & 427.53 & 378 & & \\
\hline
\end{tabular}

Note. ${ }^{* *} p<.01$ 
Table 5. Summary of ANOVA: Differences between White/Caucasian and NonWhite/Non-Caucasian Participants

\begin{tabular}{|c|c|c|c|c|c|}
\hline Post-Test Measures & & $\begin{array}{l}\text { Sum of } \\
\text { Squares }\end{array}$ & df & $\begin{array}{l}\text { Mean } \\
\text { Square }\end{array}$ & $F$ \\
\hline \multirow[t]{3}{*}{ CFIP } & Between Groups & .52 & 1 & .52 & 1.68 \\
\hline & Within Groups & 89.03 & 286 & .31 & \\
\hline & Total & 89.55 & 287 & & \\
\hline \multirow[t]{3}{*}{ Procedural Justice } & Between Groups & .37 & 1 & .37 & .64 \\
\hline & Within Groups & 164.61 & 287 & .57 & \\
\hline & Total & 164.98 & 288 & & \\
\hline \multirow[t]{3}{*}{ Distributive Justice } & Between Groups & .32 & 1 & .32 & .38 \\
\hline & Within Groups & 244.07 & 290 & .84 & \\
\hline & Total & 244.39 & 291 & & \\
\hline \multirow[t]{3}{*}{ Test-Taking Motivation } & Between Groups & .51 & 1 & .51 & .63 \\
\hline & Within Groups & 236.80 & 290 & .82 & \\
\hline & Total & 237.31 & 291 & & \\
\hline \multirow[t]{3}{*}{ Litigation Intentions } & Between Groups & 10.57 & 1 & 10.57 & $11.16^{* *}$ \\
\hline & Within Groups & 276.41 & 292 & .95 & \\
\hline & Total & 286.98 & 293 & & \\
\hline \multirow[t]{3}{*}{ Recommendation Intentions } & Between Groups & 1.72 & 1 & 1.72 & 1.49 \\
\hline & Within Groups & 334.97 & 290 & 1.16 & \\
\hline & Total & 336.69 & 291 & & \\
\hline \multirow[t]{3}{*}{ Job Acceptance Intentions } & Between Groups & 2.78 & 1 & 2.78 & 3.29 \\
\hline & Within Groups & 243.41 & 288 & .85 & \\
\hline & Total & 246.19 & 289 & & \\
\hline \multirow[t]{3}{*}{ Purchasing Intentions } & Between Groups & .72 & 1 & .72 & 1.00 \\
\hline & Within Groups & 207.96 & 290 & .72 & \\
\hline & Total & 208.68 & 291 & & \\
\hline
\end{tabular}

Note. ${ }^{* *} p<.01$ 
Table 6. Means, Standard Deviations, and Intercorrelations of Study Control Variables and Study Scales

\begin{tabular}{|c|c|c|c|c|c|c|c|c|c|c|c|c|}
\hline & & $M$ & $\mathrm{SD}$ & 1 & 2 & 3 & 4 & 5 & 6 & 7 & 8 & 9 \\
\hline \multicolumn{13}{|c|}{ Control Variables } \\
\hline 1 & Age & 25.36 & 7.38 & -- & & & & & & & & \\
\hline 2 & Internet Experience & 7.83 & 1.59 & -.08 & -- & & & & & & & \\
\hline 3 & Internet Knowledge & 7.16 & 1.75 & -.04 & $.76^{* *}$ & -- & & & & & & \\
\hline 4 & $\begin{array}{l}\text { Electronic Application } \\
\text { Preference }\end{array}$ & 3.17 & 1.23 & .08 & .05 & .04 & $(.96)$ & & & & & \\
\hline \multicolumn{13}{|c|}{ Study Scales } \\
\hline 5 & Procedural Justice & 2.45 & .75 & -.07 & .10 & .04 & .04 & $(.86)$ & & & & \\
\hline 6 & Distributive Justice & 2.18 & .92 & -.06 & $.12 *$ & .06 & .01 & $.59 * *$ & $(.91)$ & & & \\
\hline 7 & $\begin{array}{l}\text { Test-Taking } \\
\text { Motivation }\end{array}$ & 3.58 & .89 & .06 & $.13 *$ & $.12 *$ & $.13^{*}$ & $.37 * *$ & $.26^{* *}$ & $(.93)$ & & \\
\hline 8 & Litigation Intentions & 2.51 & .98 & -.09 & .04 & .08 & $-.12 *$ & $-.19 * *$ & -.05 & $-.20 * *$ & $(.94)$ & \\
\hline 9 & $\begin{array}{l}\text { Recommendation } \\
\text { Intentions }\end{array}$ & 3.25 & 1.07 & .07 & $.12 *$ & .09 & $.13^{*}$ & $.28 * *$ & $.19^{* *}$ & $.60 * *$ & $-.27 * *$ & $(.89)$ \\
\hline
\end{tabular}


Table 6. Means, Standard Deviations, and Intercorrelations of Study Control Variables and Study Scales (Continued)

\begin{tabular}{|c|c|c|c|c|c|c|c|c|c|c|c|c|c|c|}
\hline & & $M$ & SD & 1 & 2 & 3 & 4 & 5 & 6 & 7 & 8 & 9 & 10 & 11 \\
\hline 10 & $\begin{array}{l}\text { Job Acceptance } \\
\text { Intentions }\end{array}$ & 4.19 & .93 & .04 & .04 & .04 & $.17 * *$ & $.16^{* *}$ & .09 & $.60 * *$ & $-.27 * *$ & $.59 * *$ & $(.98)$ & \\
\hline 11 & Purchasing Intentions & 3.49 & .85 & -.01 & $.12 *$ & $.16^{* *}$ & $.19 * *$ & $.33 * *$ & $.20 * *$ & $.60 * *$ & $-.27 * *$ & $.62 * *$ & $.61 * *$ & $(.83)$ \\
\hline
\end{tabular}

Note. ${ }^{* *} p<.01 .{ }^{*} p<.05 . n=271-315$. Coefficient alphas found on diagonal. Measures 2 - 3 are on a 10-point scale;

Measures 4 - 11 are on a 5-point scale. 
Table 7. Means, Standard Deviations and Intercorrelations for Study Pre-Test and Post-Test Measures

\begin{tabular}{|c|c|c|c|c|c|c|c|c|c|c|c|c|}
\hline & & $M$ & $S D$ & 1 & 2 & 3 & 4 & 6 & 7 & 8 & 9 & 10 \\
\hline \multicolumn{13}{|c|}{ Pre-Test Measures } \\
\hline 1 & Belief in Legal Protection & 3.71 & .84 & $(.87)$ & & & & & & & & \\
\hline 2 & $\begin{array}{l}\text { Test-Taking Motivation } \\
\text { (VIEMS) }\end{array}$ & 4.13 & .64 & $.30^{* *}$ & $(.90)$ & & & & & & & \\
\hline 3 & Organizational Intentions & 3.97 & .62 & $.37 * *$ & $.55^{* *}$ & $(.86)$ & & & & & & \\
\hline 4 & Litigation Intentions & 2.26 & .78 & $-.26 * *$ & $-.18 * *$ & $-.23 * *$ & $(.89)$ & & & & & \\
\hline \multicolumn{13}{|c|}{ Post-Test Measures } \\
\hline 6 & Belief in Legal Protection & 3.58 & .98 & $.72 * *$ & $.15^{*}$ & $.25^{* *}$ & $-.25 * *$ & $(.93)$ & & & & \\
\hline 7 & $\begin{array}{l}\text { Test-Taking Motivation } \\
\text { (VIEMS) }\end{array}$ & 3.58 & .89 & $.17 * *$ & $.29 * *$ & $.29 * *$ & $-.17 * *$ & $.43 * *$ & $(.93)$ & & & \\
\hline 8 & Organizational Intentions & 3.64 & .81 & $.21^{* *}$ & $.18^{* *}$ & $.56^{* *}$ & $-.28 * *$ & $.47 * *$ & $.65^{* *}$ & $(.91)$ & & \\
\hline 9 & Litigation Intentions & 2.51 & .98 & $-.18 * *$ & -.09 & $-.13^{*}$ & $.64^{* *}$ & $-.25 * *$ & $-.20 * *$ &.$\overline{-}$ & (.94) & \\
\hline 10 & Privacy Concerns (CFIP) & 4.21 & .55 & .06 & $.13^{*}$ & $.13^{*}$ & .00 & .07 & .06 & .07 & .02 & $(.91)$ \\
\hline
\end{tabular}


Table 7. Means, Standard Deviations and Intercorrelations for Study Pre-Test and Post-Test Measures (Continued)

\begin{tabular}{|c|c|c|c|c|c|c|c|c|c|c|c|c|c|}
\hline & & $M$ & $S D$ & 1 & 2 & 3 & 4 & 6 & 7 & 8 & 9 & 10 & 11 \\
\hline 11 & Procedural Justice & 2.45 & .75 & .07 & -.07 & -.03 & -.11 & $.28 * *$ & $.37 * *$ & $.30 * *$ & $-.19 * *$ & -.11 & $(.86)$ \\
\hline 12 & Distributive Justice & 2.18 & .92 & -.02 & $-.16^{* *}$ & -.06 & -.01 & .11 & $.26^{* *}$ & $.20 * *$ & -.05 & $-.18 * *$ & $.59 * *$ \\
\hline 13 & Overall Fairness Perceptions & 2.35 & .67 & .04 & $-.13 *$ & -.05 & -.08 & $.23 * *$ & $.36^{* *}$ & $.29 * *$ & $-.15^{*}$ & $-.15 * *$ & $.93 * *$ \\
\hline 14 & SPJS & 2.34 & .67 & .09 & .01 & .11 & -.05 & $.30 * *$ & $.40 * *$ & $.34 * *$ & $-.14^{*}$ & $-.13 *$ & $.72 * *$ \\
\hline 15 & CFIP Subscale: Collection & 4.19 & .77 & -.06 & .06 & .04 & .03 & -.06 & .04 & -.03 & .03 & $.75^{* *}$ & $.16^{* *}$ \\
\hline 16 & CFIP Subscale: Errors & 3.72 & .82 & .09 & .05 & .00 & .04 & .10 & .01 & .01 & .06 & $.71 * *$ & .01 \\
\hline 17 & $\begin{array}{l}\text { CFIP Subscale: Unauthorized } \\
\text { Secondary Use }\end{array}$ & 4.62 & .65 & $.13 *$ & $.19^{* *}$ & $.29 * *$ & -.11 & $.14^{*}$ & .08 & $.20 * *$ & -.09 & $.69 * *$ & -.09 \\
\hline 18 & $\begin{array}{l}\text { CFIP Subscale: Improper } \\
\text { Access }\end{array}$ & 4.34 & .70 & .06 & $.13 *$ & $.11^{*}$ & .00 & .06 & .09 & .06 & .02 & $.85 * *$ & -.06 \\
\hline
\end{tabular}

Note. ${ }^{* * p}<.001 .{ }^{*} p<.05 . n=271-315$. Coefficient alphas found on diagonal. All measures are on a 5-point scale.

VIEMS $=$ Valence, Instrumentality, Expectancy Motivation Scale.

CFIP $=$ Concern for Information Privacy Scale. SPJS = Selection Procedural Justice Scale. 
Table 7. Means, Standard Deviations and Intercorrelations for Study Pre-Test and Post-Test Measures

\begin{tabular}{|c|c|c|c|c|c|c|c|c|}
\hline & & 12 & 13 & 14 & 15 & 16 & 17 & 18 \\
\hline 12 & Distributive Justice & $(.91)$ & & & & & & \\
\hline 13 & Overall Fairness Perceptions & $.85 * *$ & $(.90)$ & & & & & \\
\hline 14 & SPJS & $.57 * *$ & $.74 * *$ & $(.90)$ & & & & \\
\hline 15 & CFIP Subscale: Collection & $-.18 * *$ & $-.19 * *$ & $-.21 * *$ & $(.91)$ & & & \\
\hline 16 & CFIP Subscale: Errors & -.08 & -.03 & .09 & $.26^{* *}$ & $(.85)$ & & \\
\hline 17 & $\begin{array}{l}\text { CFIP Subscale: Unauthorized } \\
\text { Secondary Use }\end{array}$ & $-.14^{*}$ & $-.12 *$ & $-.15 * *$ & $.36^{* *}$ & $.26^{* *}$ & $(.91)$ & \\
\hline 18 & CFIP Subscale: Improper Access & $-.15 * *$ & $-.11 *$ & $-.15 * *$ & $.61 * *$ & $.50 * *$ & $.52 * *$ & $(.92)$ \\
\hline \multicolumn{9}{|c|}{$\begin{array}{l}\text { Note. }{ }^{* *} p<.001 .{ }^{*} p<.05 . n=271-315 . \text { Coefficient alphas found on diagonal in parentheses. All measures are on a 5-point } \\
\text { scale. }\end{array}$} \\
\hline
\end{tabular}


Table 8. Summary of Hierarchical Regression Results for Hypotheses 1-3

\begin{tabular}{|c|c|c|c|c|c|c|c|c|c|}
\hline Model & Variable & Adjusted $R^{2}$ & $R^{2}$ Change & F Change & $B$ & $S E B$ & $\beta$ & $t$ & $p$ \\
\hline \multirow[t]{4}{*}{1} & & .02 & .03 & $3.64 *$ & & & & & \\
\hline & (Constant) & & & & 1.75 & .23 & & 7.80 & .00 \\
\hline & Ethnic Background & & & & .08 & .10 & .05 & .81 & .42 \\
\hline & Internet Experience & & & & .07 & .03 & .15 & 2.57 & .01 \\
\hline \multirow[t]{7}{*}{2} & & .03 & .02 & 1.75 & & & & & \\
\hline & (Constant) & & & & 1.58 & .24 & & 6.68 & .00 \\
\hline & Ethnic Background & & & & .08 & .10 & .05 & .77 & .44 \\
\hline & Internet Experience & & & & .07 & .03 & .15 & 2.58 & .01 \\
\hline & Control Condition & & & & .16 & .09 & .11 & 1.78 & .08 \\
\hline & Excuse Explanation Condition & & & & .07 & .10 & .05 & .67 & .50 \\
\hline & Privacy Justification Condition & & & & .17 & .10 & .11 & 1.66 & .10 \\
\hline \multirow[t]{8}{*}{3} & & .04 & .02 & $5.89^{*}$ & & & & & \\
\hline & (Constant) & & & & 2.38 & .40 & & 5.90 & .00 \\
\hline & Ethnic Background & & & & .06 & .10 & .03 & .58 & .56 \\
\hline & Internet Experience & & & & .07 & .03 & .15 & 2.54 & .01 \\
\hline & Control Condition & & & & .16 & .09 & .11 & 1.89 & .06 \\
\hline & Excuse Explanation Condition & & & & .08 & .10 & .05 & .75 & .46 \\
\hline & Privacy Justification Condition & & & & .15 & .10 & .10 & 1.47 & .14 \\
\hline & Privacy Concerns & & & & -.18 & .08 & -.14 & -2.43 & .02 \\
\hline
\end{tabular}

Note. Dependent variable $=$ Overall Fairness Perceptions. $n=281 .{ }^{*} p<.05$. 
Table 8. Summary of Hierarchical Regression Results for Hypotheses 1-3 (Continued)

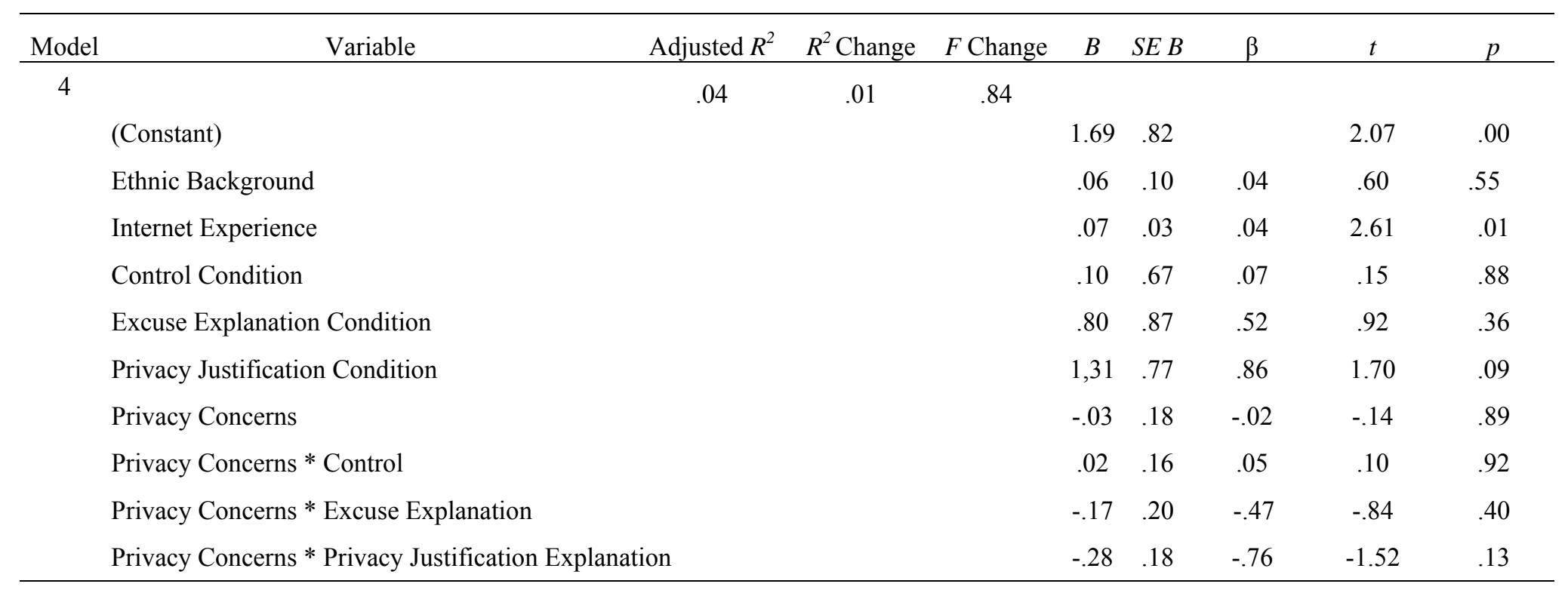

Note. Dependent variable $=$ Overall Fairness Perceptions. $n=281 .{ }^{*} p<.05$. 
Table 9. Summary of Hierarchical Regression Results for Research Question 1

\begin{tabular}{|c|c|c|c|c|c|c|c|c|c|}
\hline Model & Variable & Adjusted $R^{2}$ & $R^{2}$ Change & $F$ Change & $B$ & $S E B$ & $\beta$ & $t$ & $p$ \\
\hline \multirow[t]{4}{*}{1} & & .02 & .03 & $3.64 *$ & & & & & \\
\hline & (Constant) & & & & 1.75 & .23 & & 7.80 & .00 \\
\hline & Ethnic Background & & & & .08 & .10 & .05 & .81 & .42 \\
\hline & Internet Experience & & & & .07 & .03 & .15 & 2.57 & .01 \\
\hline \multirow[t]{7}{*}{2} & & .03 & .02 & 1.75 & & & & & \\
\hline & (Constant) & & & & 1.58 & .24 & & 6.68 & .00 \\
\hline & Ethnic Background & & & & .08 & .10 & .05 & .77 & .44 \\
\hline & Internet Experience & & & & .07 & .03 & .15 & 2.58 & .01 \\
\hline & Control Condition & & & & .16 & .09 & .12 & 1.78 & .08 \\
\hline & Excuse Explanation Condition & & & & .07 & .10 & .05 & .67 & .50 \\
\hline & Privacy Justification Condition & & & & .17 & .10 & .11 & 1.66 & .10 \\
\hline \multirow[t]{11}{*}{3} & & .05 & .04 & $2.84^{*}$ & & & & & \\
\hline & (Constant) & & & & 2.48 & .42 & & 5.98 & .00 \\
\hline & Ethnic Background & & & & .09 & .10 & .06 & .92 & .36 \\
\hline & Internet Experience & & & & .07 & .03 & .15 & 2.51 & .01 \\
\hline & Control Condition & & & & .15 & .09 & .10 & 1.74 & .08 \\
\hline & Excuse Explanation Condition & & & & .07 & .10 & .04 & .65 & .51 \\
\hline & Privacy Justification Condition & & & & .12 & .10 & .08 & 1.20 & .23 \\
\hline & CFIP: Collection & & & & -.16 & .07 & -.18 & -2.42 & .02 \\
\hline & CFIP: Errors & & & & .03 & .06 & .03 & .46 & .65 \\
\hline & CFIP: Unauthorized Secondary Us & & & & -.10 & .08 & -.09 & -1.24 & .22 \\
\hline & CFIP: Improper Access & & & & .04 & .09 & .04 & .43 & .67 \\
\hline
\end{tabular}

Note. Dependent variable $=$ Overall Fairness Perceptions. $n=281 .{ }^{*} p<.05$. CFIP $=$ Concern for Information Privacy Scale 
Table 10. Summary of Hierarchical Regression Results for Hypothesis $4 a$

\begin{tabular}{|c|c|c|c|c|c|c|c|c|c|}
\hline Model & Variable & Adjusted $R^{2}$ & $R^{2}$ Change & $F$ Change & $B$ & SE B & $\beta$ & $t$ & $p$ \\
\hline \multirow[t]{7}{*}{1} & & .06 & .02 & 1.49 & & & & & \\
\hline & (Constant) & & & & 2.71 & .32 & & 8.42 & .00 \\
\hline & Ethnic Background & & & & -.45 & .14 & -.19 & -3.32 & .00 \\
\hline & Internet Experience & & & & .035 & .036 & .057 & .983 & .33 \\
\hline & Control Condition & & & & -.05 & .12 & -.02 & -.40 & .69 \\
\hline & Excuse Explanation Condition & & & & -.28 & .14 & -.13 & -1.99 & .05 \\
\hline & Privacy Justification Condition & & & & -.06 & .14 & -.03 & -.43 & .67 \\
\hline \multirow[t]{8}{*}{2} & & .07 & .02 & $5.22 *$ & & & & & \\
\hline & (Constant) & & & & 3.02 & .35 & & 8.70 & .00 \\
\hline & Ethnic Background & & & & -.43 & .13 & -.19 & -3.24 & .00 \\
\hline & Internet Experience & & & & .05 & .04 & .07 & 1.27 & .21 \\
\hline & Control Condition & & & & -.02 & .12 & -.01 & -.15 & .88 \\
\hline & Excuse Explanation Condition & & & & -.27 & .14 & -.13 & -1.91 & .06 \\
\hline & Privacy Justification Condition & & & & -.03 & .14 & -.02 & -.24 & .81 \\
\hline & Overall Fairness Perceptions & & & & -.18 & .08 & -.13 & -2.28 & .02 \\
\hline
\end{tabular}

Note. Dependent variable $=$ Litigation Intentions. $n=287 .{ }^{*} p<.05 .{ }^{* *} p<.01$. 
Table 11. Summary of Hierarchical Regression Results for Hypothesis $4 b$

\begin{tabular}{|c|c|c|c|c|c|c|c|c|c|}
\hline Model & Variable & Adjusted $R^{2}$ & $R^{2}$ Change & $F$ Change & $B$ & $S E B$ & $\beta$ & $t$ & $p$ \\
\hline \multirow[t]{7}{*}{1} & & .11 & .01 & 1.14 & & & & & \\
\hline & (Constant) & & & & 3.10 & .27 & & 11.40 & .00 \\
\hline & Ethnic Background & & & & .16 & .11 & .09 & 1.42 & .16 \\
\hline & Internet Experience & & & & .05 & .03 & .09 & 1.47 & .14 \\
\hline & Control Condition & & & & .09 & .10 & .05 & .90 & .37 \\
\hline & Excuse Explanation Condition & & & & .13 & .12 & .08 & 1.11 & .27 \\
\hline & Privacy Justification Condition & & & & -.05 & .12 & -.03 & -.42 & .67 \\
\hline \multirow[t]{8}{*}{2} & & .09 & .08 & $23.70 * *$ & & & & & \\
\hline & (Constant) & & & & 2.58 & .28 & & 9.12 & .00 \\
\hline & Ethnic Background & & & & .14 & .11 & .07 & 1.29 & .20 \\
\hline & Internet Experience & & & & .03 & .03 & .05 & .88 & .38 \\
\hline & Control Condition & & & & .04 & .10 & .02 & .41 & .68 \\
\hline & Excuse Explanation Condition & & & & .10 & .11 & .06 & .91 & .37 \\
\hline & Privacy Justification Condition & & & & -.10 & .11 & -.06 & -.85 & .39 \\
\hline & Overall Fairness Perceptions & & & & .31 & .07 & .28 & 4.87 & .00 \\
\hline
\end{tabular}


Table 12. Summary of Hierarchical Regression Results for Hypothesis $4 \mathrm{C}$

\begin{tabular}{|c|c|c|c|c|c|c|c|c|c|}
\hline Model & Variable & Adjusted $R^{2}$ & $R^{2}$ Change & F Change & $B$ & $S E B$ & $\beta$ & $t$ & $p$ \\
\hline \multirow[t]{7}{*}{1} & & .02 & .02 & 2.13 & & & & & \\
\hline & (Constant) & & & & 2.83 & .30 & & 9.49 & .00 \\
\hline & Ethnic Background & & & & .10 & .12 & .05 & .79 & .43 \\
\hline & Internet Experience & & & & .07 & .03 & .12 & 1.99 & .05 \\
\hline & Control Condition & & & & .21 & .12 & .11 & 1.89 & .06 \\
\hline & Excuse Explanation Condition & & & & .15 & .13 & .08 & 1.15 & .25 \\
\hline & Privacy Justification Condition & & & & -.05 & .13 & -.02 & -.36 & .72 \\
\hline \multirow[t]{8}{*}{2} & & .15 & .12 & $35.08 * *$ & & & & & \\
\hline & (Constant) & & & & 2.16 & .30 & & 7.11 & .00 \\
\hline & Ethnic Background & & & & .06 & .12 & .03 & .49 & .63 \\
\hline & Internet Experience & & & & .04 & .03 & .07 & 1.23 & .22 \\
\hline & Control Condition & & & & .13 & .10 & .07 & 1.28 & .20 \\
\hline & Excuse Explanation Condition & & & & .12 & .12 & .06 & 1.02 & .31 \\
\hline & Privacy Justification Condition & & & & -.11 & .12 & -.06 & -.91 & .36 \\
\hline & Overall Fairness Perceptions & & & & .42 & .07 & .34 & 5.92 & .00 \\
\hline
\end{tabular}


Table 13. Mediation Tests: Litigation Intentions

\begin{tabular}{|c|c|c|c|c|}
\hline Variable & $\beta$ & $S E$ & $t$ & $p$ \\
\hline $\begin{array}{l}\text { Fairness Perceptions regressed on } \\
\text { Privacy Concerns }\end{array}$ & -.18 & .08 & -2.43 & .02 \\
\hline $\begin{array}{l}\text { Litigation Intentions regressed on } \\
\text { Fairness Perceptions }\end{array}$ & -.18 & .08 & -2.15 & .03 \\
\hline $\begin{array}{l}\text { Litigation Intentions regressed on } \\
\text { Privacy Concerns* (Total Effect) }\end{array}$ & .03 & .11 & .26 & .80 \\
\hline $\begin{array}{l}\text { Litigation Intentions regressed on } \\
\text { Privacy Concerns* (Direct Effect) }\end{array}$ & -.01 & .12 & -.05 & .96 \\
\hline & & $S E$ & $z$ & $p$ \\
\hline
\end{tabular}

Indirect effect and significance using normal distribution

\begin{tabular}{ccccc} 
Sobel & & .02 & 1.61 & .11 \\
\hline & $M$ & $S E$ & LL 95\%CI & UL 95\%CI \\
\hline
\end{tabular}

Bootstrap Results for indirect effect

Effect
.03 .03 $-.002$ .10

Note. $n=281$. Unstandardized regression coefficients are reported. Bootstrap sample size $=5,000 . \mathrm{LL}=$ lower limit; $\mathrm{CI}=$ confidence interval; $\mathrm{UL}=$ upper limit.

*Analysis performed while controlling for previously identified control variables and study conditions. 
Table 14. Mediation Tests: Organizational Intentions

\begin{tabular}{lcccc}
\hline Variable & $\beta$ & $S E$ & $t$ & $p$ \\
\hline & & & & \\
& & & & \\
$\begin{array}{l}\text { Fairness Perceptions regressed on } \\
\text { Privacy Concerns }\end{array}$ & -.17 & .08 & -2.31 & .02 \\
$\begin{array}{l}\text { Organizational Intentions regressed on } \\
\text { Fairness Perceptions }\end{array}$ & .36 & .07 & 5.36 & .00 \\
$\begin{array}{l}\text { Organizational Intentions regressed on } \\
\text { Privacy Concerns* (Total Effect) }\end{array}$ & .12 & .09 & 1.40 & .16 \\
$\begin{array}{l}\text { Organizational Intentions regressed on } \\
\text { Privacy Concerns* (Direct Effect) }\end{array}$ & .19 & 0.08 & 2.21 & .03 \\
\hline & & & & \\
\hline
\end{tabular}

Indirect effect and significance using normal distribution

Sobel

\begin{tabular}{ccccc} 
Sobel & & .03 & 2.12 & .03 \\
\hline$M$ & SE & LL 95\%CI & UL 95\%CI \\
\hline
\end{tabular}

Bootstrap Results for indirect effect

Effect $-.06$ .03 $-.14$ $-.01$

Note. $n=274$. Unstandardized regression coefficients are reported. Bootstrap sample size $=5,000 . \mathrm{LL}=$ lower limit; $\mathrm{CI}=$ confidence interval; $\mathrm{UL}=$ upper limit.

*Analysis performed while controlling for previously identified control variables and study conditions. 
Table 15. Mediation Tests: Test-Taking Motivation

Variable

$\beta$

$S E$

$t$

$p$

Fairness Perceptions regressed on

Privacy Concerns

$-.18 \quad .08$

$-2.43$

.02

Test-Taking Motivation regressed on

Fairness Perceptions

.45

.07

6.30

.00

Test-Taking Motivation regressed on Privacy Concerns* (Total Effect)

Test-Taking Motivation regressed on Privacy Concerns* (Direct Effect) .18 .09

1.97

.05

\begin{tabular}{lllll}
\hline$S E$ & $z$ & $p$ \\
\hline
\end{tabular}

Indirect effect and significance using normal distribution

Sobel

\begin{tabular}{ccccc} 
& .04 & 2.27 & .02 \\
\hline & $M$ & $S E$ & LL 95\%CI & 95\% \\
\hline
\end{tabular}

Bootstrap Results for indirect effect

Effect $-.83$ .04 $-.16$ $-.01$

Note. $n=281$. Unstandardized regression coefficients are reported. Bootstrap sample size $=5,000 . \mathrm{LL}=$ lower limit; $\mathrm{CI}=$ confidence interval; UL $=$ upper limit.

*Analysis performed while controlling for previously identified control variables and study conditions. 
Table 16. Summary of Hierarchical Regression Results for Research Question 2

\begin{tabular}{|c|c|c|c|c|c|c|c|c|c|}
\hline Model & Variable & Adjusted $R^{2}$ & $R^{2}$ Change & F Change & $B$ & $S E B$ & $\beta$ & $t$ & $p$ \\
\hline \multirow[t]{7}{*}{1} & & .004 & .02 & 1.22 & & & & & \\
\hline & (Constant) & & & & 4.33 & .19 & & 23.46 & .00 \\
\hline & Ethnic Background & & & & -.11 & .08 & -.08 & -1.35 & .18 \\
\hline & Internet Experience & & & & -.01 & .02 & -.02 & -.29 & .77 \\
\hline & Control Condition & & & & .05 & .07 & .04 & .72 & .47 \\
\hline & Excuse Explanation Condition & & & & .04 & .08 & .03 & .49 & .62 \\
\hline & Privacy Justification Condition & & & & -.11 & .08 & -.09 & -1.36 & .18 \\
\hline \multirow[t]{8}{*}{2} & & .01 & .01 & 1.95 & & & & & \\
\hline & (Constant) & & & & 4.12 & .24 & & 17.35 & .00 \\
\hline & Ethnic Background & & & & -.11 & .08 & -.08 & -1.40 & .16 \\
\hline & Internet Experience & & & & -.01 & .02 & -.02 & -.30 & .76 \\
\hline & Control Condition & & & & .05 & .07 & .04 & .68 & .50 \\
\hline & Excuse Explanation Condition & & & & .04 & .08 & .03 & .50 & .62 \\
\hline & Privacy Justification Condition & & & & -.11 & .08 & -.10 & -1.42 & .16 \\
\hline & Belief in Legal Protection & & & & .06 & .04 & .08 & 1.40 & .16 \\
\hline
\end{tabular}


Table 17. Pre-Test and Post-Test Means by Study Condition for Distal Outcome Variables

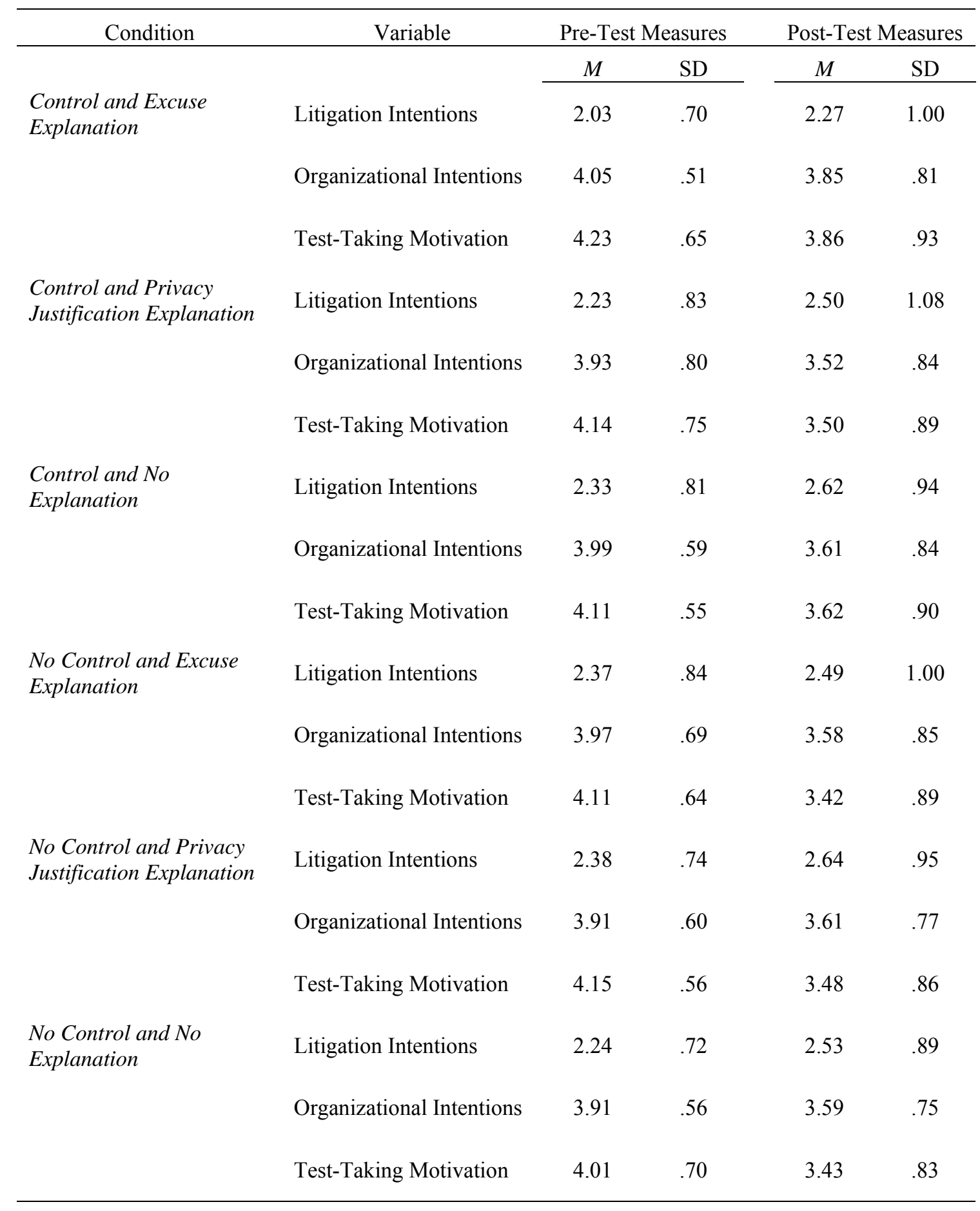

Note. $n=36-71$. 
Figure 1. Model of Applicants' Reactions to Employment Selection Systems

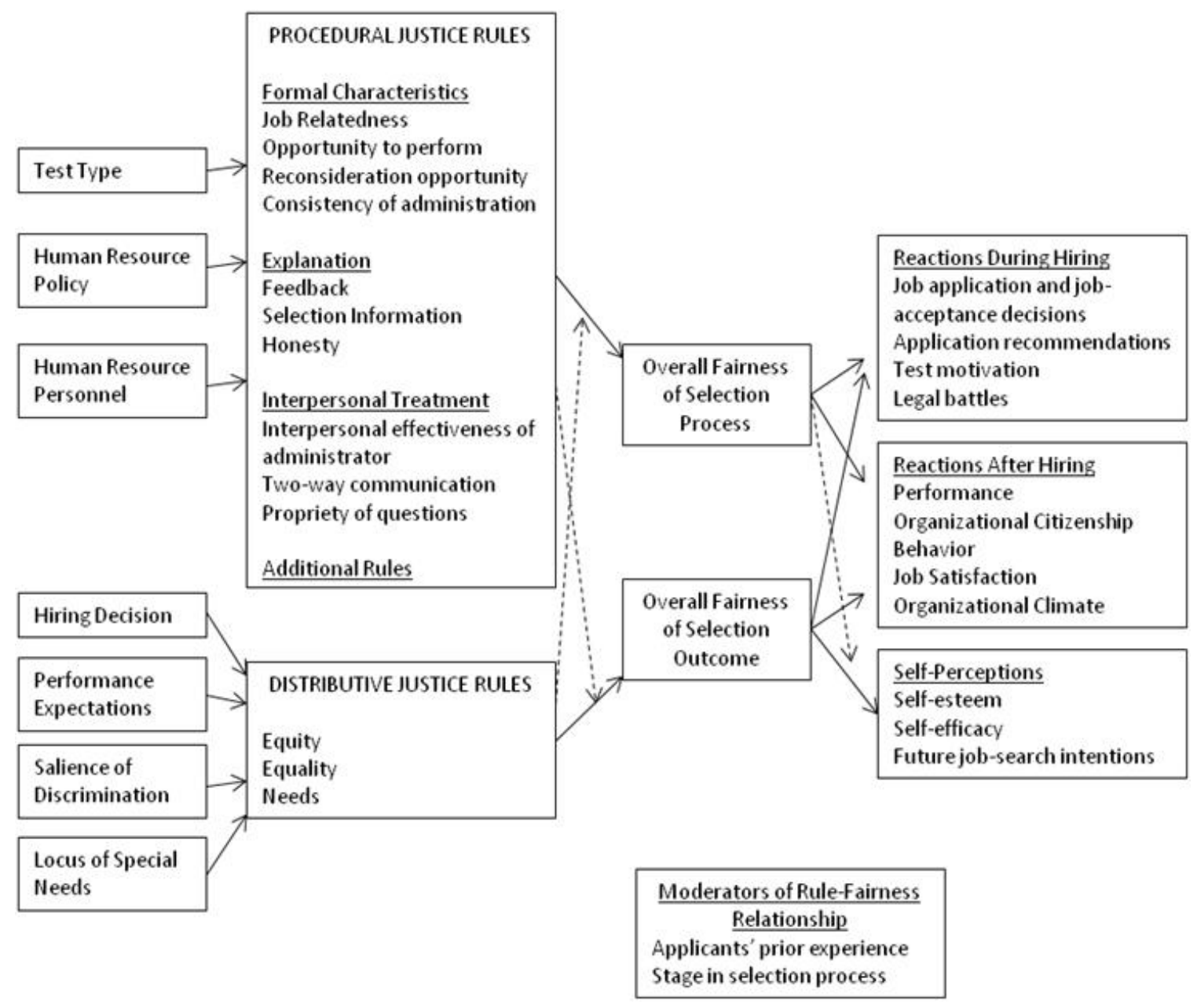

Gilliland (1993, p.700) 
Figure 2. Hypothesized Overall Model of Applicant Reactions to Privacy Concerns

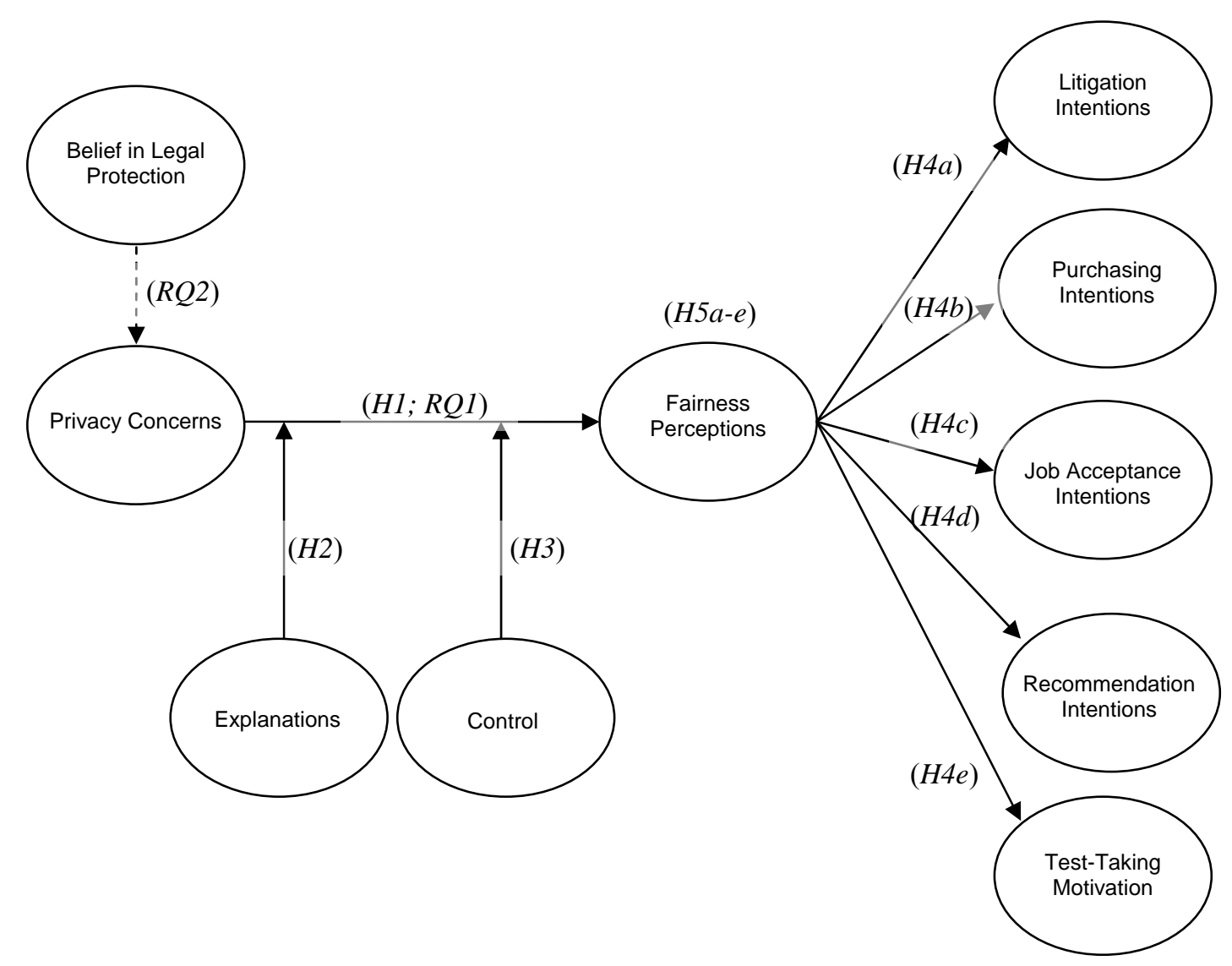


Figure 3. Hypothesized (Revised) Overall Model of Applicant Reactions to Privacy

\section{Concerns}

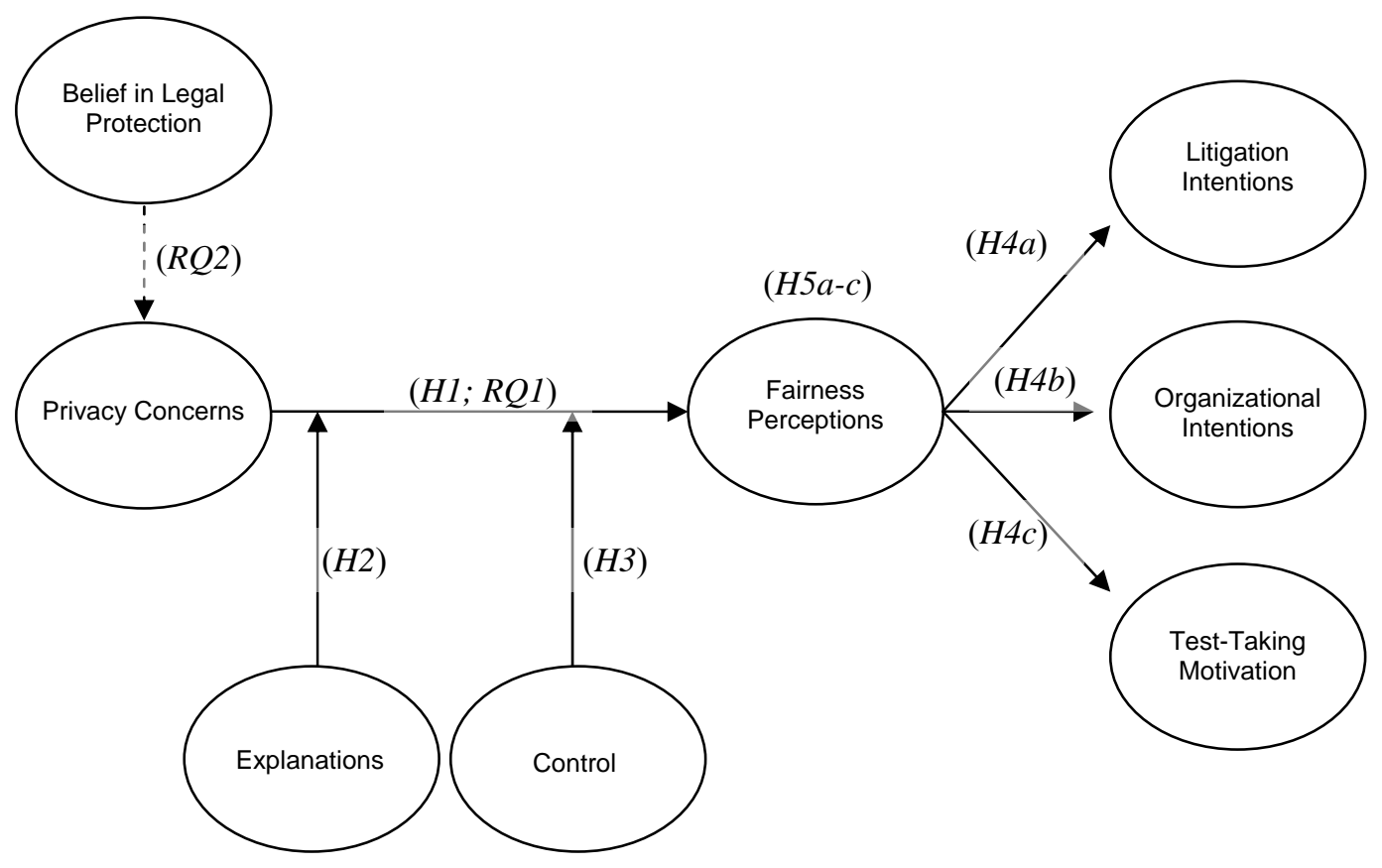


Figure 4. Hypothesized Relationship between Privacy Concerns and Explanations in Predicting Fairness Perceptions

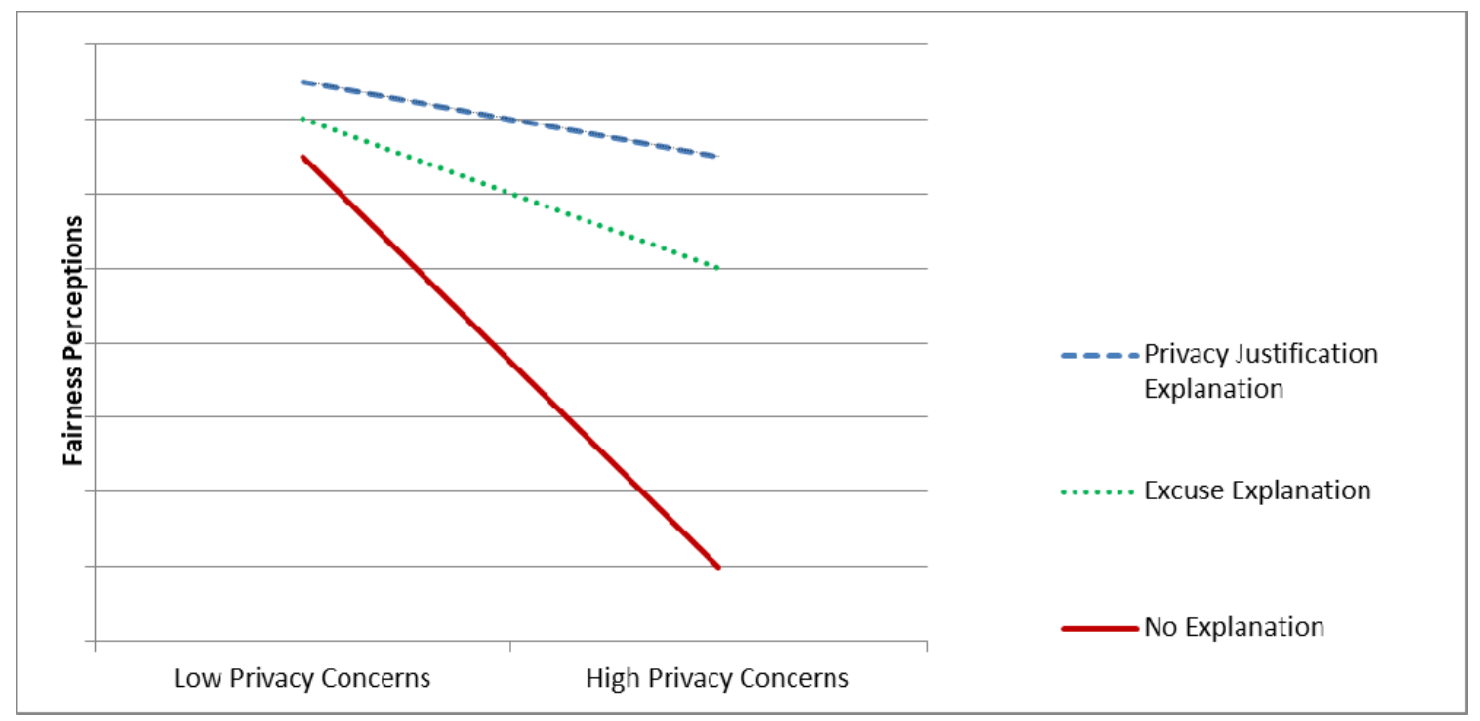


Figure 5. Hypothesized Relationship between Privacy Concerns and Control in Predicting Fairness Perceptions

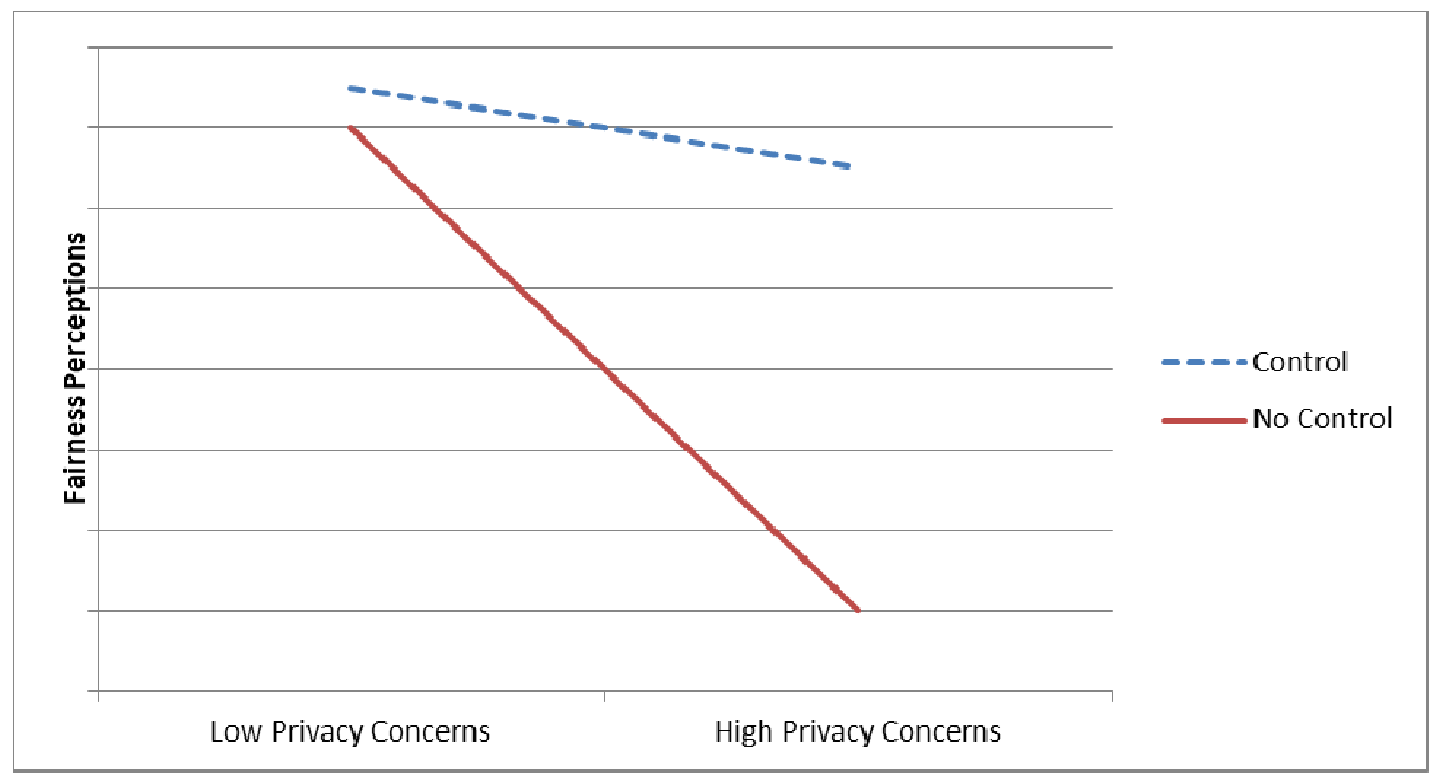


Figure 6. Results of Hypothesis Tests

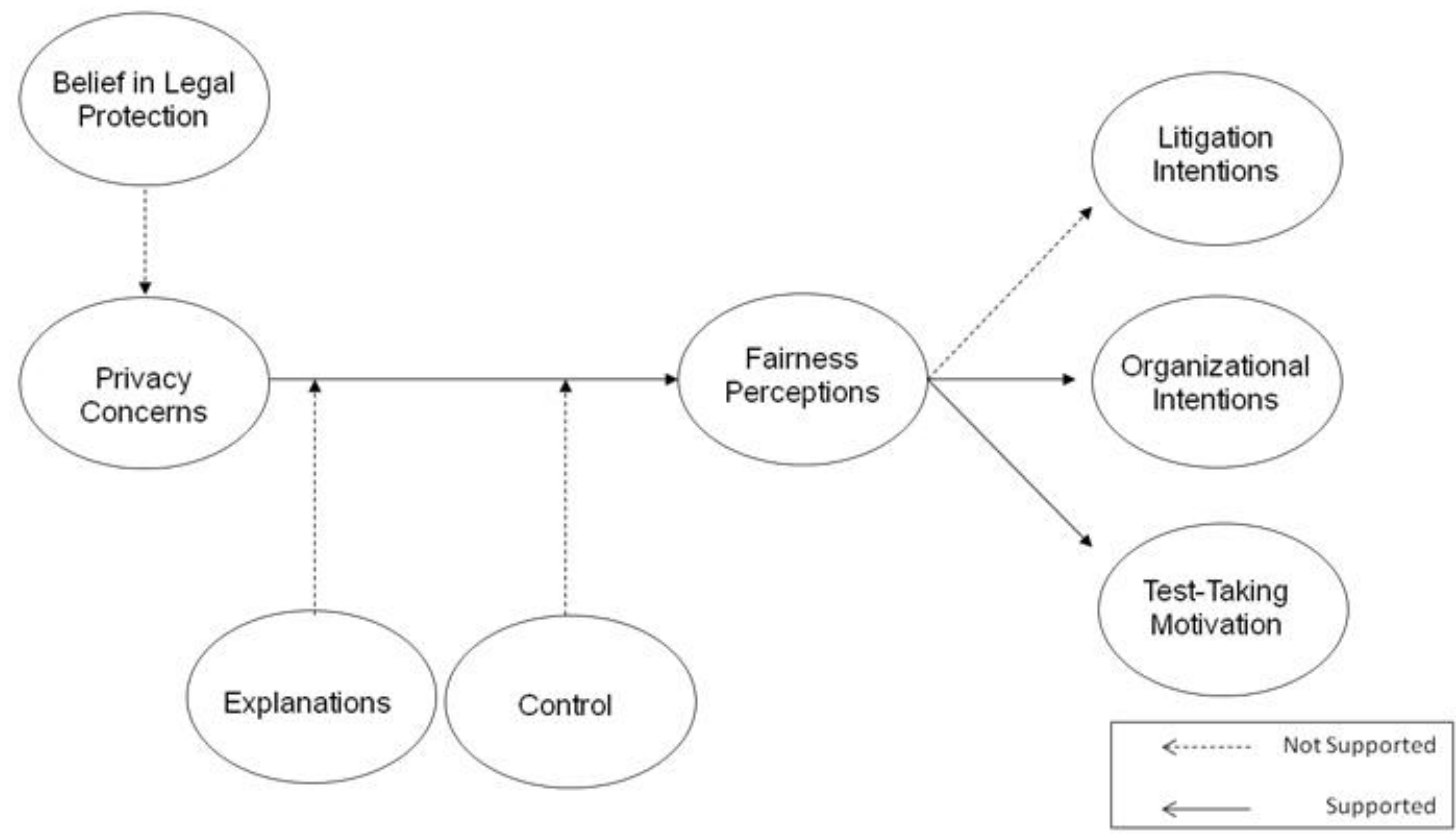


Figure 7. Confirmatory Factor Model for Test-Taking Motivation

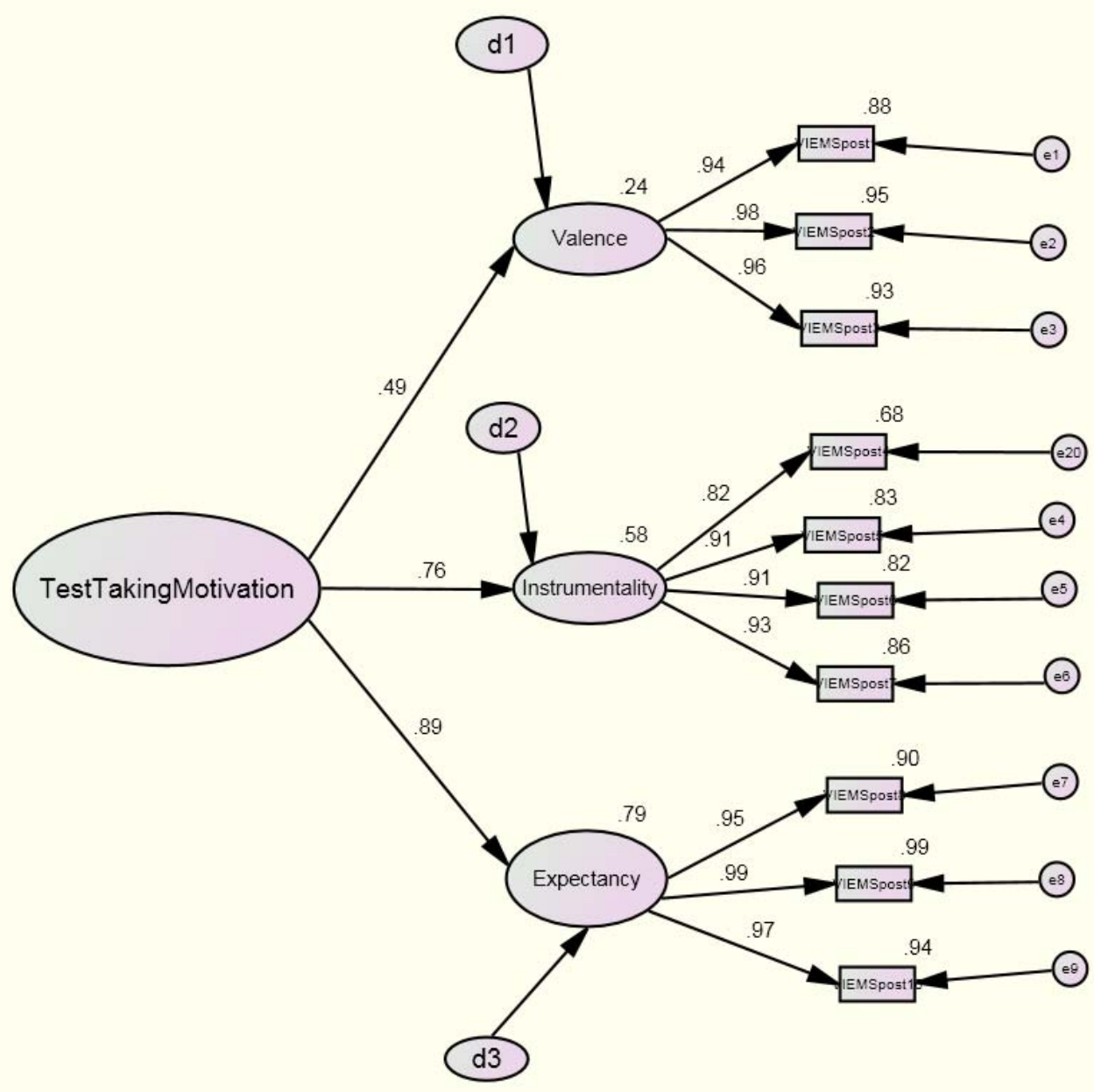


Figure 8. Confirmatory Factor Model for Organizational Intentions

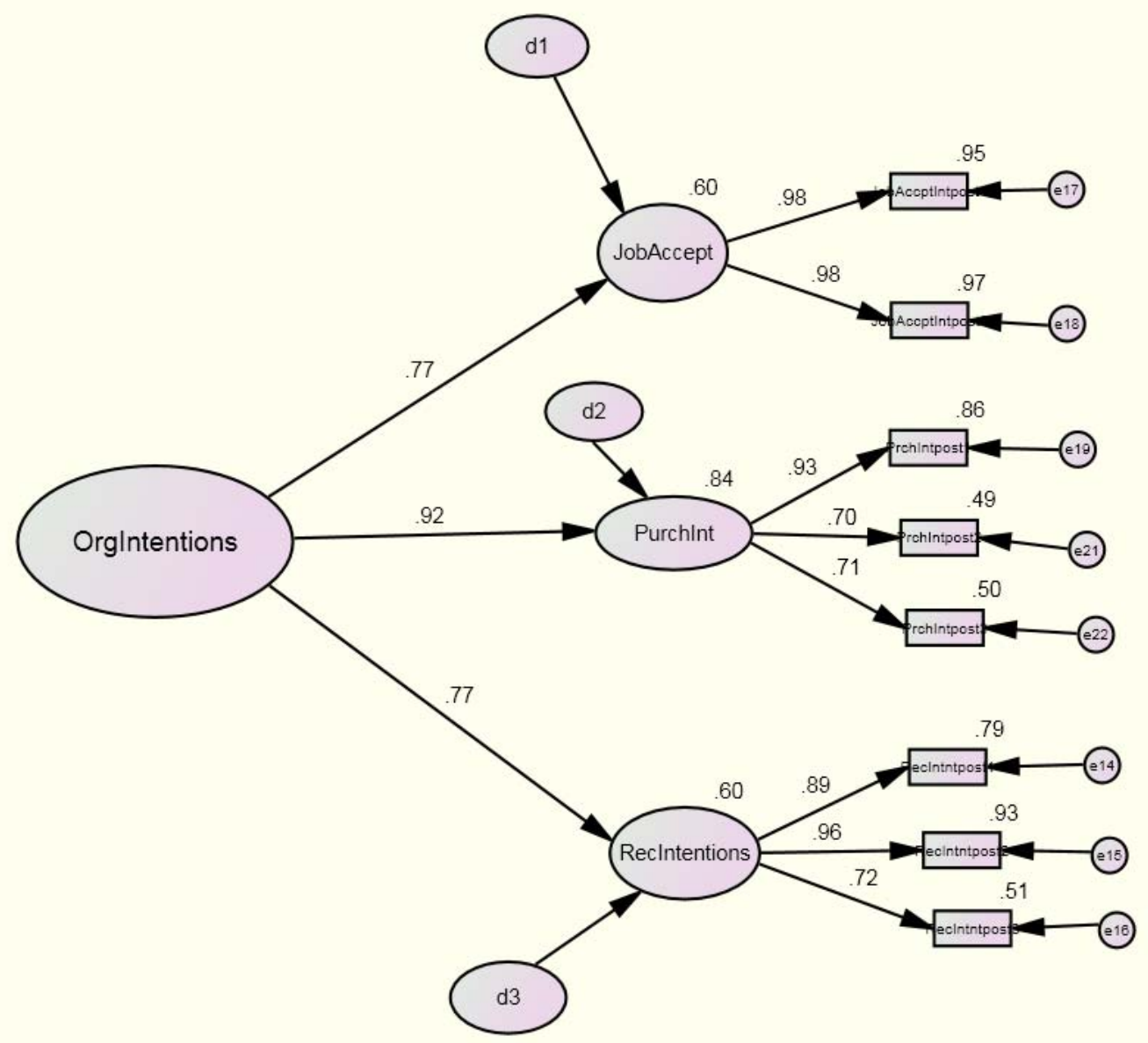


Figure 9. Confirmatory Factor Model for Litigation Intentions

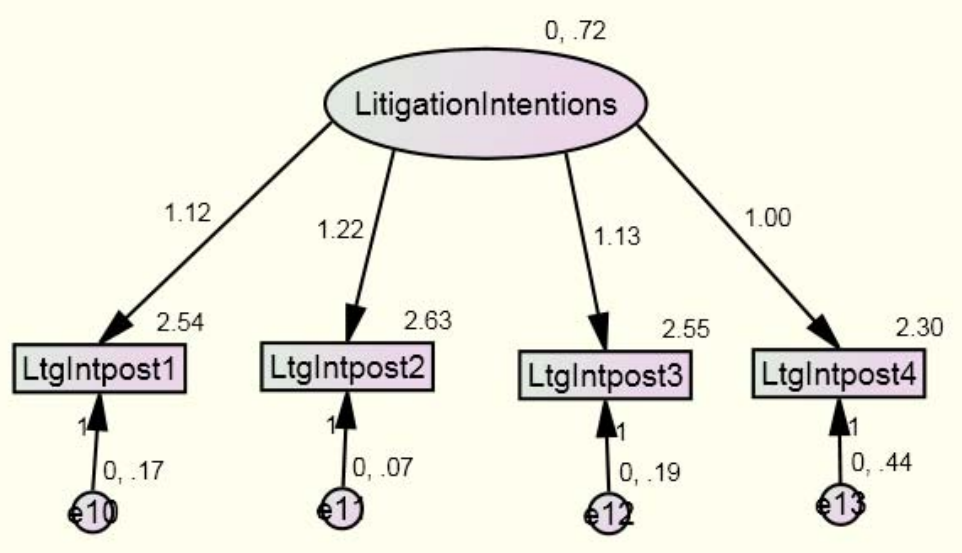


Figure 10. Initial Confirmatory Factor Model for Overall Fairness Perceptions

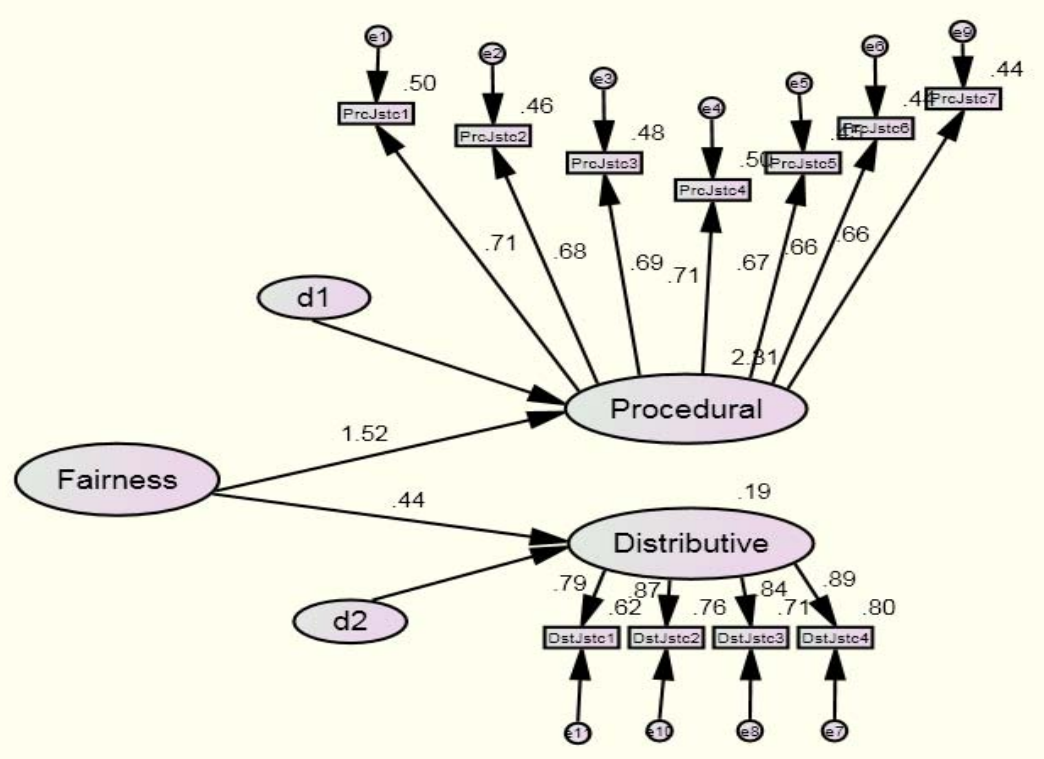


Figure 11. Final Confirmatory Factor Model for Overall Fairness Perceptions

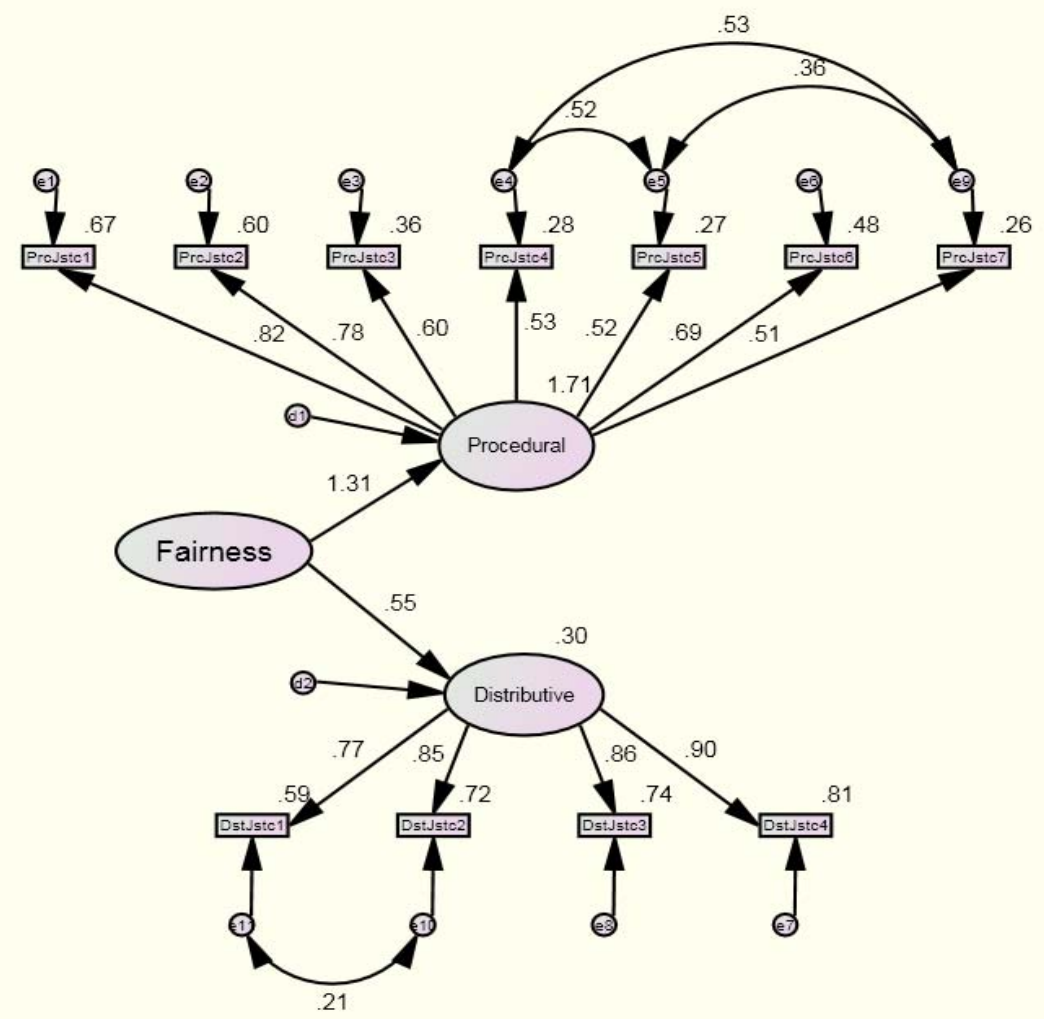


Figure 12. Confirmatory Factor Model for Dimensions of the Concerns for Information Privacy Scale

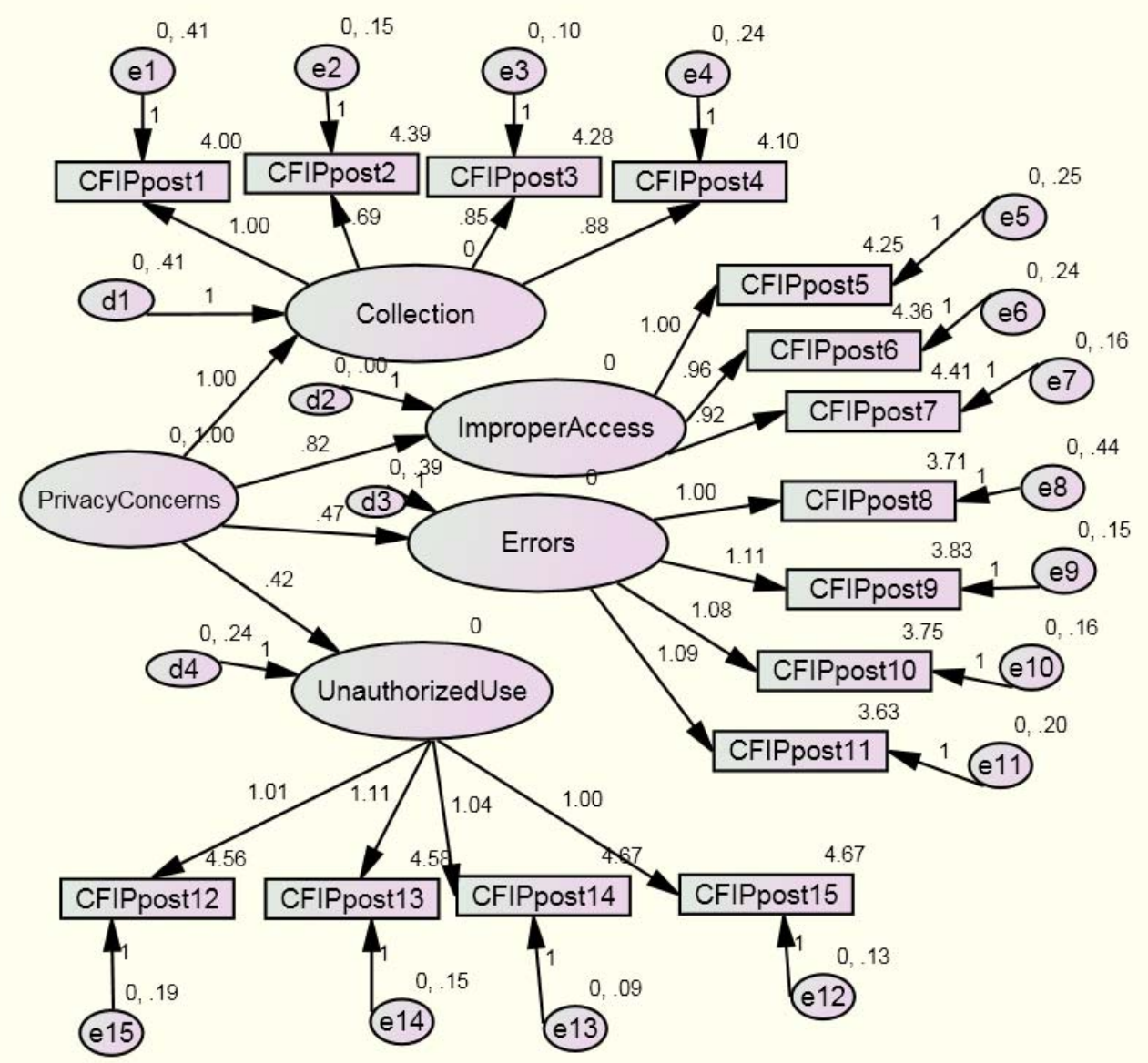


Figure 13. Relationship between Privacy Concerns and Explanations in Predicting Test-

\section{Taking Motivation}

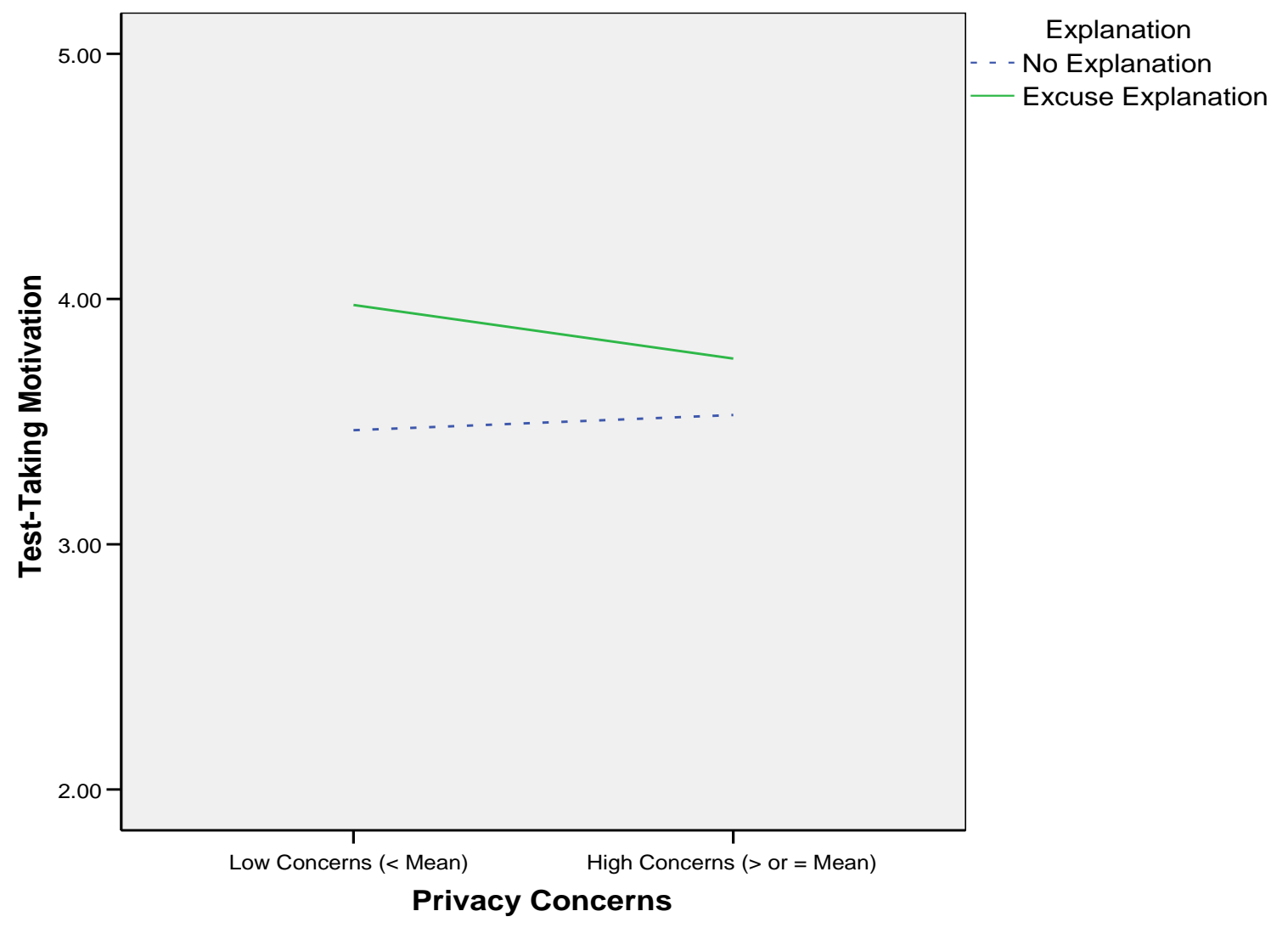




\section{REFERENCES}

Acohido, B. (2009, January 28). Hackers hit Monster.com's customer data again; Second time in 6 months that job seekers' info stolen. USA Today, p. B3.

Adams, J.S. (1965). Inequity in social exchange. In L. Berkowitz (Ed.), Advances in experimental social psychology (Vol. 2, pp. 267-299). New York, NY: Academic Press.

Aguinas, H., Beaty, J.C., Boik, R.J., \& Pierce, C.A. (2005). Effect size and power in assessing moderating effects of variables using multiple regression: A 30-year review. Journal of Applied Psychology, 90, 94-107.

Alge, B.J. (2001). Effects of computer surveillance on perceptions of privacy and procedural justice. Journal of Applied Psychology, 86, 797-804.

Alder, G.S., \& Ambrose, M.L. (2005). An examination of the effect of computerized performance monitoring feedback on monitoring fairness, performance, and satisfaction. Organizational Behavior and Human Decision Processes. 97, 161177.

Alder, G.S., Noel, T.W, \& Ambrose, M.L. (2006). Clarifying the effects of internet monitoring on job attitudes: The mediating role of employee trust. Information \& Management, 43, 894-903.

Ambrose, M.L., Hess, R.L., \& Ganesan, S. (2007). The relationship between justice and attitudes: An examination of justice efects on event and system-related attitudes. Organizational Behavior and Human Decision Processes, 103, 21-36. 
Ambrose, M.L., \& Cropanzano, R. (2003). A Longitudinal analysis of organizational fairness: An examination of reactions to tenure and promotion decisions. Journal of Applied Psychology, 88, 266-275.

Ambrose, M.L., \& Alder, G.S. (2000). Designing, implementing, and utilizing computerized performance monitoring: Enhancing organizational justice. Research in Personnel and Human Resources Management, 18, 187-219.

Argyris, C. (1960). Understanding organisational behaviour. Homewood, Illinois: Dorsey Press.

Arvey, R.D., \& Sackett, P.R. (1993). Fairness in selection: Current developments and perspectives. In N. Schmitt, \& W. Borman (Eds.), Personnel Selection, (pp. 171202). San Francisco: Jossey-Bass.

Arvey, R.D., Strickland, W., Drauden, G., \& Martin, C. (1990). Motivational components of test taking. Personnel Psychology, 43, 695-716.

Bauer, T.N., Maertz, C.P., Dolen, M.R., \& Campion, M.A. (1998). Longitudinal assessment of applicant reactions to employment testing and test outcome feedback. Journal of Applied Psychology, 83, 892-903.

Bauer, T.N., Truxillo, D.M., Tucker, J.S., Weathers, V., Bertolino, M., Erdogan, B., \& Campion, M.A. (2006). Selection in the information age: The impact of privacy concerns and computer experience on applicant reactions. Journal of Management, 32, 601-621. 
Bauer, T.N., Truxillo, D.M., Sanchez, F.J., Craig, J.M., Ferrara, P., \& Campion, M.A. (2001). Applicant Reactions to Selection: Development of the selection procedural justice scale (SPJS). Personnel Psychology, 54, 387-419.

Bellman, S., Johnson, E., Kobrin, S., \& Lohse, G. (2004). International differences in information privacy concerns: A global survey of consumers. Information Society, 20, 313-324.

Bennett, C., Ordonez, L.D., \& Gilliland, S.W. (2003). Grade expectations: The effects of expectations on fairness and satisfaction perceptions. Journal of Behavioral Decision Making, 16, 375-395.

Bertolino, M., \& Steiner, D.D. (2007). Fairness reactions to selection methods: An Italian study. International Journal of Selection and Assessment, 15, 197-205.

Bies, R.J. (2001). Interactional (in)justice: The sacred and the profane. In R. Cropanzano \& J. Greenberg (Eds.), Advances in organizational justice (pp. 89-118). Stanford, CA: Stanford University Press.

Bies, R.J., \& Moag, J.S. (1986). Interactional justice: Communication criteria for fairness. In B. Sheppard (Ed.), Research on negotiation in organizations (Vol. 1, pp. 43-55). Greenwich, CT: JAI Press.

Bies, R.J., \& Shapiro, D.L. (1988). Voice and justification: Their influence on procedural fairness judgments. Academy of Management Journal, 31, 676-685.

Brockner, J., Paruchuri, S., Idson, L.C., \& Higgins, E.T. (2002). Regulatory focus and the probability estimates of conjunctive and disjunctive events. Organizational Behavior and Human Decision Processes, 87, 5-24. 
Bordonaro, G. (2009, June 9). Aetna named in security-breach lawsuit. Hartford Business Journal Online. Retrieved from http://www.hartfordbusiness.com/news9190.html Bureau of Labor Statistics (BLS). (2009). Labor force statistics from the current population survey. Retrieved October 2, 2009 from http://www.bls.gov/cps/demographics.htm

Cawley, B., Keeping, L., \& Levy, P. (1998). Participation in the performance appraisal process and employee reactions: A meta-analytic review of field investigations. Journal of Applied Psychology, 83, 615-633.

Chan, D., Schmitt, N., DeShon, R.P., Clause, C.S., \& Delbridge, K. (1997). Reactions to cognitive ability tests: The relationships between race, test performance, face validity perceptions, and test-taking motivation. Journal of Applied Psychology, $82,300-310$.

Chambliss, D.F., \& Schutt, R.K. (2006). Making sense of the social world: Methods of investigation. Thousand Oaks, CA: Pine Forge Press.

Civil Rights Act of 1964, Title VII, 42 U.S.C. $§ 2000 \mathrm{e}-2(\mathrm{~h})$.

Cleeton, G.U., Kraft, M.A., \& Royster, R.F. (1946). Selection and employment of transit operating personnel. New York: The American Transit Association.

Colquitt, J.A., Conlon, D.E., Wesson, M.J., Porter, C.O.L.H., \& Yee NG, K. (2001). Justice at the millennium: A meta-analytic review of 25 years of organizational justice research. Journal of Applied Psychology, 86, 245-445. 
Connerley, M.L., Arvey, R.D., Gilliland, S.W., Mael, F.A., Paetzold, R.L., \& Sackett, P.R. (2001). Selection in the workplace: Whose rights prevail? Employee Responsibilities \& Rights Journal, 13, 1-13

Cropanzano, R., \& Ambrose, M.L. (2001). Procedural and distributive justice are more similar than you think: A monistic perspective and research agenda. In J. Greenberg \& R. Cropanzano (Eds.), Advance in Organizational Justice, (pp. 119151). Stanford, CA: Stanford University Press.

Cropanzano, R., \& Schminke, M. (2001). Using social justice to build effective work groups. In M. Turner (Ed), Groups at work: Advances in theory and research (pp.143-171). Hillsdale, NJ: Erlbaum.

Dinev, T. \& Hart, P. (2004). Internet privacy concerns and their antecedents measurement validity and a regression model. Behaviour \& Information Technology, 6, 413-422.

Directive on Privacy and Electronic Communications. (2002). Office Journal of the European Committees, DIRECTIVE 2002/58/EC.

Downs, C.W. (1969). Perceptions of the selection interview. Personnel Administration, $32,8-23$.

Donovan, J.J. (2001). Work motivation. In N. Anderson, D.S. Ones, H.K. Sinangil, \& C. Viswesvaran (Eds.), Handbook of industrial, work, and organizational psychology (Vol. 2, pp. 53-76). Thousand Oaks, CA: Sage Publications.

Dukes v. Wal-Mart Stores, Inc., 04-16688, 04-16720 (9 $9^{\text {th }}$ Cir. 2010). 
Eddy, E.R., Stone, D.L., \& Stone-Romero, E.F. (1999). The effects of information management policies on reactions to human resource systems: An integration of privacy and procedural justice perspectives. Personnel Psychology, 52, 335-358.

Electronic Privacy Information Center. (2011). Facebook privacy. Retrieved January 31, 2011 from http://epic.org/privacy/facebook/

Equal Employment Opportunity Commission. (2010). EEO reports / surveys. Retrieved from http://www.eeoc.gov/employers/reporting.cfm

Farrar, D.E., \& Glauber, R.R. (1967). Multicollinearity in regression analysis: The problem revisited. The Review of Economics and Statistics, 49, 92-107.

Faul, F., Erdfelder, E., Buchner, A., \& Lang, A.G. (2009). Statistical power analyses using $\mathrm{G}^{*}$ Power 3.1: Tests for correlation and regression analyses. Behavior Research Methods, 41, 1149-1160.

Festinger, L. (1954). A theory of social comparison processes. Human Relations, 7, 117140.

Folger, R. (1987). Relative deprivation and referent cognitions: Reformulating the preconditions of resentment. In J.C. Masters, \& W.P. Smith (Eds.), Social comparison, social justice, and relative deprivation: Theoretical, empirical, and policy perspectives (pp. 183 -215). Hillsdale, NJ: Erlbaum.

Folger, R., \& Cropanzano, R. (2001). Fairness theory: Justice as accountability. In J. Greenberg \& R. Cropanzano (Eds.), Advances in organizational justice (pp. 155). Stanford, CA: Stanford University Press.

Frank, T. (2007, May 8). Probe launched into missing TSA computer drive; 
Data include information on 100,000 agency employees. USA Today, p. A13. Gatewood, R.D., Feild, H.S., \& Barrick, M.R. (2007). Human resource selection (6 ${ }^{\text {th }}$ ed.). Cincinnati, $\mathrm{OH}$ : Southwestern College Publishing Company. Gilliland, S.W. (1993). The perceived fairness of selection systems: An organizational justice perspective. Academy of Management Review, 18, 694-734.

Gilliland, S.W. (1994). The effects of procedural and distributive justice on reactions to a selection system. Journal of Applied Psychology, 79, 691-701.

Gilliland, S.W., Groth, M., Baker IV, R.C., Dew, A.F., Polly, L.M., \& Langdon, J.C. (2001). Improving applicants' reactions to rejection letters: An application of fairness theory. Personnel Psychology, 54, 669-703.

Goldman, B.M. (2003). The application of referent cognitions theory to legal-claiming by terminated employees. Journal of Management, 29, 705-728.

Goldman, B.M., Paddock, E., \& Cropanzano, R. (2004). A transformational model of legal-claiming. Journal of Managerial Issues, 16, 417.

Greenberg, J. (1993). The social side of fairness: Interpersonal and informational classes of organizational justice. In R. Cropanzano (Ed.), Justice in the workplace, (pp. 79-106). Hillsdale, NJ: Erlbaum.

Greenberg, J., \& Cropanzano, R. (2001). (Ed.). Advances in organizational justice. Stanford, CA: Stanford University Press.

Guion, R.M. (1965). Personnel testing. New York: McGraw-Hill. Handler, C.A., \& Hunt, S.T. (2003). Rocket-hire buyer's guide to web-based screening \& staffing assessment systems. United States: Rocket-Hire. 
Harris, M.M. (1989). Reconsidering the employment interview: A review of recent literature and suggestions for future research. Personnel Psychology, 42, 691-726.

Harris, M.M., Van Hoye, G., \& Lievens, F. (2003). Privacy and attitudes towards internet-based selection systems: A cross-cultural comparison. International Journal of Selection and Assessment, 11, 230-236.

Hausknecht, J.P., Day, D.V., \& Thomas, S.C. (2004). Application reactions to selection procedures: An updated model and meta-analysis. Personnel Psychology, 57, 636683.

Horvath, M., Ryan, A.M., \& Stierwalt, S.L., (2000). The influence of explanation for selection test use, outcome favorability, and self-efficacy on test-taker perceptions. Organizational Behavior and Human Decision Processes, 83, 310 330.

Is your privacy important? Are you losing it? (2006). Privacy Lost. Retrieved July 15, 2007, from http://www.msnbc.msn.com/id/14850268.

Jensen, C., Potts, C., \& Jensen, C. (2005). Privacy practices of internet users: Self-reports versus observed behavior. International Journal of Computer Studies, 63, 203227.

Johnson, C. (2001, September 11). Report criticizes monster.com; privacy advocates say job-search site misuses data. The Washington Post, p. E04.

Kappauf, W.E. (1948). Review of the publication: Selection and employment of transit operating personnel. Psychological Bulletin, 45, 95-96. 
Kenny, D.A., Kashy, D.A., \& Bolger, N. (1998). Data analysis in social psychology. In D. Gilbert, S. Fiske, \& G. Lindzey (Eds.), The handbook of social psychology $\left(4 t^{h}\right.$ Ed.). (Vol. 1, pp. 233-265). Boston, MA: McGraw-Hill.

Kernan, M.C., \& Hanges, P.J. (2002). Survivor reactions to reorganization: Antecedents and consequences of procedural, interpersonal, and informational justice. Journal of Applied Psychology, 87, 916-928.

Kluger, A.N., \& Rothstein, H. (1993). The influence of selection test type on applicant reactions to employment testing. Journal of Business and Psychology, 8, 3-25.

LaHuis, D.M., Perreault , N.E., \& Ferguson, M.W. (2003). The effects of legitimizing explanations on applicants' perceptions of fairness. Journal of Applied Social Psychology, 33, 2198-2215.

Lauer, T., \& Deng, X. (2007). Building online trust through privacy practices. International Journal of Information Security, 6, 323-331.

Leventhal, G.S. (1980). What should be done with equity theory? New approaches to the study of fairness in social relationships. In K. Gergen, M. Greenberg, \& R. Willis (Eds.), Social exchange: Advances in theory and research (pp. 27-55). New York: Plenum Press.

Lind, E.A., \& Tyler, T.R. (1988). The social psychology of procedural justice. New York: Plenum.

Lind, E.A., Kray, L., \& Thompson, L. (2001). Primacy effects in justice judgments: Testing predictions from fairness heuristic theory. Organizational Behavior and Human Decision Processes, 85, 189-210. 
Lind, E.A., Kanfer, R., \& Earley, P.C. (1990). Voice, control, and procedural justice: Instrumental and noninstrumental concerns in fairness judgments. Journal of Personality and Social Psychology, 59, 952-959.

Macan, T.H., Avedon, M., Paese, M., \& Smith, D.E. (1994). The effects of applicants' reactions to cognitive ability tests and an assessment center. Personnel Psychology, 47, 715-738.

Mael, F.A. (1998). Privacy and personnel selection: Reciprocal rights and responsibilities. Employee Responsibilities and Rights Journal, 11, 187-214.

Malhotra, N.K., Kim, S.S., \& Agarwal, J. (2004). Internet users' information privacy concerns (IUIPC): The construct, the scale, and a causal model. Information Systems Research, 15, 336-355.

Masterson, S., Lewis, K., Goldman, B.M., \& Taylor, M.S. (2000). Integrating justice and social exchange: The differing effects of fair procedures and treatment on work relationships. Academy of Management Journal, 43, 738-748.

Matarazzo, J., Wiens, A., Jackson, R., \& Manaugh, T. (1970). Interviewee speech behavior under different content conditions. Journal of Applied Psychology, 54, $15-26$.

Maurer, S.D., \& Fay, C.H. (1988). The effect of situational interviews, conventional structured interviews and training on interview rating agreement. Personnel Psychology, 41, 329-344.

Mayer, D.M., \& Ployhart, R.E. (2007). Explaining away injustice: When, why, and for whom are explanations most effective? Working paper. 
MacKinnon, D.P., Warsi, G., \& Dwyer, J.H. (1995). A simulation study of mediated effect measures. Multivariate Behavioral Research, 30, 41-62.

McNall, L.A., \& Roch, S.G. (2007). Effects of electronic monitoring types on perceptions of procedural justice, interpersonal justice, and privacy. Journal of Applied Social Psychology, 37, 658-682.

Milberg, S.J., Smith, H.J., \& Burke S.J. (2000). Information privacy: Corporate management and national regulation. Organization Science, 11, 35-57.

Miniwatts Marketing Group. (2009). World internet users and population stats. Accessed September 12, 2009: http://www.internetworldstats.com/stats.htm

Nikolaou, I., \& Judge, T.A. (2007). Fairness reactions to personnel selection techniques in Greece: The role of core self-evaluations. International Journal of Selection and Assessment, 15, 206-219.

Office of Strategic Service Assessment Staff. (1948). Assessment of men: Selection of personnel for the Office of Strategic Services. New York, NY: Rinehart \& Company.

Pillai, R., Schriesheim, C.A., \& Williams, E.S. (1999). Fairness perceptions and trust as mediators for transformational and transactional leadership: A two-sample study. Journal of Management, 25, 897-933.

Ployhart, R.E., Ryan, A.M., \& Bennett, M. (1999). Explanations for selection decisions: Applicants' reactions to informational and sensitivity features of explanations. Journal of Applied Psychology, 84, 87-106. 
Ployhart, R.E., \& Ryan, A.M. (1997). Toward an explanation of applicant reactions: An examination of organizational justice and attribution frameworks. Organizational Behavior and Human Decision Processes. 72, 308-335.

Ployhart, RE., \& Ryan, A. M. (1998). Applicants' reactions to the fairness of selection procedures: The effects of positive rule violations and time of measurement. Journal of Applied Psychology, 83, 3-16.

Preacher, K.J., \& Hayes, A.F. (2008). Asymptotic and resampling strategies for assessing and comparing indirect effects in multiple mediator models. Behavior Research Methods, 40, 879-891.

Rosenbaum, B.L. (1973). Attitude toward invasion of privacy in the personnel selection process and job applicant demographic and personality correlates. Journal of Applied Psychology, 58, 333-338.

Rousseau, D. M. (1989). Psychological and implicit contracts in organizations. Employee Responsibilities and Rights Journal, 2, 121-139.

Russell, G.W. (1999). Tuning in to the conversation: Twenty-five years later. Journal of Popular Culture, 33, 123-130.

Ryan, A. M., \& Ployhart, R. E. (2000). Applicants' perceptions of selection procedures and

decisions: A critical review and agenda for the future. Journal of Management, $26,565-606$.

Rynes, S.L., \& Barber, A.E. (1990). Applicant attraction strategies: An organizational perspective. Academy of Management Review, 15, 286-310. 
Rynes, S.L., Heneman, H.G., \& Schwab, D.P. (1980). Individual reactions to organizational recruiting: A review. Personnel Psychology, 33, 529-542.

Sackett, P.R., Schmitt, N., Ellingson, J.E., \& Kabin, M.B. (2001). High-stakes testing in employment, credentialing, and higher education: Prospects in a post-affirmativeaction world. American Psychologist, 56, 302-318.

Sanchez, R.J., Truxillo, D.M., \& Bauer, T.N. (2000). Development and examination of an expectancy-based measure of test-taking motivation. Journal of Applied Psychology, 85, 339-750.

Scarborough, D., \& Somers, M.J. (2006). Neural networks in organizational research: Applying pattern recognition to the analysis of organizational behavior. Washington, DC: American Psychological Association.

Schleicher, D.J., Venkataramani, V., Morgeson, F.P., \& Campion, M.A. (2006). So you didn't get the job ... now what do you think? Examining opportunity-to-perform fairness perceptions. Personnel Psychology, 59, 559-590.

Schmitt, N., \& Coyle, B.W. (1976). Applicant decisions in the employment interview. Journal of Applied Psychology, 61, 184-192.

Schmit, M.J., \& Ryan, A.M. (1992). Test-taking dispositions: A missing link? Journal of Applied Psychology, 77, 629-637.

Schmit, M.J., \& Ryan, A.M. (1997). Applicant withdrawal: The role of test-taking attitudes and racial differences. Personnel Psychology, 50, 855-876.

Scott, M.B., \& Lyman, S.M. (1968). Accounts. American Sociological Review, 33, 4662. 
Shaw, J.C., Wild, E., \& Colquitt, J.A. (2003). To justify or excuse? A meta-analytic review of the effects of explanations. Journal of Applied Psychology, 88, 444458.

Simons, T., \& Roberson, Q. (2003). Why managers should care about fairness: The effects of aggregate justice perceptions on organizational outcomes. Journal of Applied Psychology, 88, 432-443.

Sinar, E.F., \& Reynolds, D.H. (2001). Applicant reactions to internet-based selection techniques. Paper presented at the Annual Conference for the Society of Industrial Organizational Psychology, San Diego, California.

Smith, M. (1948). Cautions concerning the use of the Taylor-Russell tables in employee selection. Journal of Applied Psychology, 32, 595-600.

Smither, J.W., Reilly, R.R., Millsap, R.E., Pearlman, K., \& Stoffey, R.W. (1993). Applicant reactions to selection procedures. Personnel Psychology, 46, 49-75.

Sobel, M.E. (1982). Asymptotic intervals for indirect effects in structural equations models. In S. Leinhart (Ed.), Sociological methodology 1982 (pp.290-312). San Francisco: Jossey-Bass.

Society for Industrial/Organizational Psychology (2003). Principles for the Validation and Use of Personnel Selection Procedures ( $4^{\text {th }}$ ed.). Bowling Green, OH: Society for Industrial/Organizational Psychology.

Soroka v. Dayton Hudson Corp., I Cal.Rptr.2d 77, 1991 Cal.App. LEXIS 1241 (Cal.Ct.App. 1st Dist.1991). 
Steiner, D.D., \& Gilliland, S.W. (1996). Fairness reactions to personnel selection techniques in France and the United States. Journal of Applied Psychology, 81, $134-141$.

Stewart, K. A., \& Segars, A. H. (2002). An empirical examination of the concern for information privacy instrument. Information Systems Research 13, 36-49.

Stone, E.F., Gueutal, H.G., Gardner, D.G., \& McClure, S. (1983). A field experiment comparing information-privacy values, beliefs, and attitudes across several types of organizations. Journal of Applied Psychology, 68, 459-468.

Stone, E.F., \& Stone, D.L. (1990). Privacy in organizations: Theoretical issues, research findings, and protection mechanisms. Research in Personnel and Human Resources Management, 8, 349-411.

Stone-Romero, E.F., Stone, D.L., \& Hyatt, D. (2003). Personnel selection procedures and invasion of privacy. Journal of Social Issues, 59, 343-368.

Sullivan, B. (2006). 'La difference' is stark in EU, U.S. privacy laws. Retrieved July 15, 2007, from http://www.msnbc.msn.com/id/15221111.

Sullivan, B. (2006). Privacy under attack, but does anybody care? Retrieved July 15, 2007, from http://www.msnbc.msn.com/id/15289066.

Sullivan, L. \& Arnold, D.W. (2000). Invasive questions lead to legal challenge, settlement and use of different test. The Industrial-Organizational Psychologist, 38, 142143. 
Taylor, S.T. (2008). Security breaches, labyrinth of domestic \& international laws heating up privacy law. Of Counsel, 27, 1-19.

Taylor, M.S., \& Bergman, T.J. (1987). Organizational recruitment activities and applicants' reactions at different stages of the recruitment process. Personnel Psychology, 40, 231-285.

Taylor, M.S., \& Sniezek, J.A. (1984). The college recruitment interview: Topical content and applicant reactions. Journal of Occupational Psychology, 57, 157-168,

Thagard, P. (2010). Cognitive science. In R. Frodeman, J.T. Klein, \& C. Mitcham (Eds.), The Oxford handbook of interdisciplinarity. (pp. 234-245). New York, NY: Oxford University Press.

Thibaut, J., \& Walker, L. (1975). Procedural justice: A psychological analysis. Hillsdale, NJ: Erlbaum.

Thibodeaux, H.F., \& Kudisch, J.D. (2003). The relationship between applicant reactions, the likelihood of complaints, and organization attractiveness. Journal of Business and Psychology, 15, 247-257.

Thronson, H., \& Thomas, W. (1968). Student opinions of the placement process. Journal of College Placement, 29, 80-84.

Truxillo, D.M., Bodner, T., Bertolino, M., Bauer, T.N., \& Yonce, C.A. (2009). Effects of explanations on applicant reactions: A meta-analytic review. International Journal of Selection and Assessment, 17, 346-361. 
Truxillo, D.M., Bauer, T.N., Campion, M.A., \& Paronto, M.E. (2002). Selection fairness information and applicant reactions: A longtitudinal field study. Journal of Applied Psychology, 87, 1020-1031.

Tyler, T.R. (1987). Conditions leading to value expressive effect in judgments of procedural justice: A test of four models. Journal of Personality and Social Psychology, 52, 333-344.

Tyler, T.R., \& Bies, R.J. (1990). Beyond formal procedures: The interpersonal context of procedural justice. In J. Carroll (Ed.), Applied social psychology and organizational settings (pp. 77-98). Hillsdale, NJ: Lawrence Erlbaum.

Tyler, T.R., Rasinski, K.A., \& Spodick, N. (1985). Influence of voice on satisfaction with leaders: Exploring the meaning of process control. Journal of Personality and Social Psychology, 48, 72-81.

Tyler, T.R., \& Lind, E.A. (1992). A relational model of authority in groups. In L. Berkowitz (Ed.), Advances in experimental social psychology (pp. 115-192). San Diego, CA: Academic Press.

van den Bos, K., Vermunt, R., \& Wilke, H.A.M. (1997). Procedural and distributive justice: What is fair depends more on what comes first than on what comes next. Journal of Applied Psychology, 72, 95-104.

Vroom, V.H. (1964). Work and motivation. New York, NY: Wiley.

Waung, M., \& Brice, T.S. (2000). Communicating negative hire decisions to applicants: Fulfilling psychological contracts. Journal of Business and Psychology, 15, 247263. 
Yonce, C.A. (2005). Rejection in the job selection process: The effects of information and sensitivity. Unpublished Master's Thesis, Orlando, FL: University of Central Florida.

Zhang, N., \& Zhao, W. (2008). Privacy protection against malicious adversaries in distributed information sharing systems. IEEE Transactions on Knowledge \& Data Engineering, 20, 1028-1033. 


\section{APPENDIX A}

Measures

Pre-Test Measures

Test-taking Motivation, Valence, Instrumentality, and Expectancy Motivation Scale (VIEMS)

Valence

1. I would like to be hired for this job.

2. It would be good to have a job with The Smith Company.

3. I want to get a job with The Smith Company. Instrumentality

4. If you do well on this test, you have a good chance of being hired.

5. I think you will be hired if you get a high test score.

6. How well you do on this test will affect whether you are hired.

7. The higher your test score, the better your chances of getting hired. Expectancy

8. You can get a good score on this test if you put some effort into it.

9. If you try to do your best on this test, you can get a high score.

10. If you concentrate and try hard you can get a high test score. Belief in Legal Protection (Privacy Regulations)

1. I believe that laws and regulations protect my privacy.

2. I believe that laws and regulations that protect my privacy are enforced. 
3. If the privacy of my personal information was in question, I believe that laws and regulations would help me.

\section{Electronic Application Preference}

1. I would prefer to complete an Internet-based job application rather than a paperbased job application.

2. Applying for a job using the Internet is more appealing to me than completing a paper- based application.

3. Using the Internet to apply for a job is better than completing a paper-based application.

\section{Recommendation Intentions}

1. I intend to encourage others to apply for a job with this company.

2. I intend to tell others that this is a good company to apply for a job.

3. I intend to apply for a job here again if I am not offered a job today.

\section{Job Acceptance Intentions}

1. If selected, I intend to accept a position with The Smith Company.

2. I would accept a position with The Smith Company.

\section{Purchasing Intentions}

1. I would shop at a store owned by the Smith Company.

2. Following my participation in the Internet-based selection process, I would be more likely to shop at the Smith Company.

3. Most shoppers would make purchases from a store owned by The Smith Company 


\section{Litigation Intentions}

1. An organization that uses an Internet-based application and test like this would likely be sued by applicants.

2. I think applicants might sue a company that used a selection process like this.

3. If Internet-based applications and tests become more widely used with job applicants, there will be an increase in the number of lawsuits against employers.

4. I would be more likely to sue an organization that used Internet-based applications and tests than one that did not.

Post-Test Measures

Concern for Information Privacy (CFIP) Scale - Adapted

\section{Collection}

1. It usually bothers me when Web sites ask me for personal information.

2. When Web sites ask me for personal information, I sometimes think twice before providing it.

3. It bothers me to give personal information to so many Web sites.

4. I'm concerned that Web sites are collecting too much personal information about me.

Improper access

5. Web sites should devote more time and effort to preventing illegal access to personal information.

6. Databases that contain personal information should be protected from illegal access - no matter how much it costs. 
7. Web sites and other companies should take more steps to make sure that hackers cannot access the personal information in their computers.

Errors

8. All the information received on Web sites should be double-checked for accuracy - no matter how much this costs.

9. Web sites should take more steps to make sure that the personal information in their files is accurate.

10. Web sites should have better procedures to correct errors in personal information.

11. Web sites should devote more time and effort to verifying the accuracy of the personal information in their databases.

Unauthorized Secondary Use

12. Web sites should not use personal information for any purpose unless it has been authorized by the individuals who provide the information.

13. When people give personal information to a Web site for some reason, the Web site should never use the information for any other reason.

14. Web sites should never sell the personal information they have collected to other Web sites.

15. Web sites should never share personal information with other Web sites or companies unless it has been authorized by the individuals who provided the information.

Selection Procedural Justice Scale (SPJS)

Structure Fairness Factor $(\alpha=.88)$ 
Job-relatedness-Predictive

1. Doing well on this test means a person can do the sales job well.

2. A person who scored well on this test will be a good salesperson. Information Known

3. I understood in advance what the testing processes would be like.

4. I knew what to expect on the test.

5. I had ample information about what the format of the test would be.

\section{Chance to Perform}

6. I could really show my skills and abilities through this test.

7. This test allowed me to show what my job skills are.

8. This test gives applicants the opportunity to show what they can really do.

9. I was able to show what I can do on this test.

Social Fairness Factor $(\alpha=.93)$

Propriety of Questions

10. The content of the test did not appear to be prejudiced.

11. The test itself did not seem too personal or private.

12. The content of the test seemed appropriate.

Job-relatedness Content Factor $(\alpha=.88) \mathrm{Th}$

Job-relatedness-Content

13. It would be clear to anyone that this test is related to the sales job.

14. The content of the test was clearly related to the sales job. 
Overall Perceptions of Procedural Justice (Fairness)

1. I was able to express my views and feelings during the Internet-based selection process.

2. I had influence over the hiring decision that was made based on the Internet-based selection process.

3. The Internet-based selection process was applied consistently.

4. The Internet-based selection process was free of bias.

5. The Internet-based selection process was based on accurate information.

6. I was able to appeal the outcome arrived at by the Internet-based selection process.

7. The Internet-based selection process upheld ethical and moral standards.

Overall Perceptions of Distributive Justice (Fairness)

1. The hiring decision reflects the effort I put into the Internet-based selection process.

2. The hiring decision was appropriate based on the answers I gave during the Internet-based selection process.

3. The hiring decision reflects my qualifications as demonstrated during the Internetbased selection process.

4. The hiring decision was justified given the information I submitted during the Internet-based selection process.

Test-taking Motivation, Valence, Instrumentality, and Expectancy Motivation Scale (VIEMS)

Valence 
1. I would like to be hired for this job.

2. It would be good to have a job with The Smith Company.

3. I want to get a job with The Smith Company. Instrumentality

4. If you do well on this test, you have a good chance of being hired.

5. I think you will be hired if you get a high test score.

6. How well you do on this test will affect whether you are hired.

7. The higher your test score, the better your chances of getting hired.

Expectancy

8. You can get a good score on this test if you put some effort into it.

9. If you try to do your best on this test, you can get a high score.

10. If you concentrate and try hard you can get a high test score.

\section{Belief in Legal Protection (Privacy Regulations)}

1. I believe that laws and regulations protect my privacy.

2. I believe that laws and regulations that protect my privacy are enforced.

3. If the privacy of my personal information was in question, I believe that laws and regulations would help me.

Electronic Application Preference

1. I would prefer to complete an Internet-based job application rather than a paperbased job application.

2. Applying for a job using the Internet is more appealing to me than completing a paper- based application. 
3. Using the Internet to apply for a job is better than completing a paper-based application.

\section{Recommendation Intentions}

1. I intend to encourage others to apply for a job with this company.

2. I intend to tell others that this is a good company to apply for a job.

3. I intend to apply for a job here again if I am not offered a job today.

\section{Job Acceptance Intentions}

1. If selected, I intend to accept a position with [company name].

2. I would accept a position with [company name].

\section{Purchasing Intentions}

1. I would shop at a store owned by the Smith Company.

2. Following my participation in the Internet-based selection process, I would be more likely to shop at the Smith Company.

3. Most shoppers would make purchases from a store owned by The Smith Company

\section{Litigation Intentions}

1. An organization that uses an Internet-based application and test like this would likely be sued by applicants.

2. I think applicants might sue a company that used a selection process like this.

3. If Internet-based applications and tests become more widely used with job applicants, there will be an increase in the number of lawsuits against employers. 
4. I would be more likely to sue an organization that used Internet-based applications and tests than one that did not.

Perceived Experience and Knowledge of the Internet

1. Please indicate the level of experience you have with the Internet on a scale of 110, where 10 indicates that you have more experience with the Internet than most others and 1 indicates that you have less experience with the Internet than most others.

2. Please indicate the level of knowledge you have of the Internet on a scale of 1-10, where 10 indicates that you have more knowledge of the Internet than most others and 1 indicates that you have less knowledge of the Internet than most others.

\section{Employment Status}

1. Please select the option that best describes your employment status:

- Employed (full-time or part-time), not actively seeking new employment

- Employed (full-time or part-time), actively seeking new employment

- Unemployed, not seeking employment

- Unemployed, seeking employment

2. If you are unemployed and seeking employment, for how many months have you been actively searching for a job?

- $\quad$ Months (fill in number)

3. If you are employed and actively seeking new employment, for how many months have you been actively searching for a job?

- $\quad$ Months (fill in number)

\section{Demographics}

1. Please provide your age: 
2. Where did you complete the online assessment?

- At your home/place of residence

- At a friend/family member's home/place of residence

- At work or school

- At a public library

- At a place of business (coffee shop, café, etc.)

- Other

\section{Facebook Questions}

1. Do you have a Facebook or other Internet-Based Social-Networking Tool:

- Yes

- No

2. If you use Facebook or another Internet-Based Social Networking Tool, please describe your level of privacy restrictions:

- $\quad$ Least Restrictive (Everyone Can See/Find Me)

- Moderately Restrictive (Selected People Can See Me/ Find Me)

- Very Restrictive (Few to No People Can See Me/Find Me)

Manipulation Check

1. An explanation was provided to me regarding the purpose or use of the selection procedure.

2. I had the opportunity to decline the release of my personal information to The Smith Company.

3. I was not offered a job with The Smith Company. 


\section{APPENDIX B}

\section{Recruitment Form}

Dear Potential Participant:

We are conducting a study on peoples' reactions to aspects of Internet-based job applications and selection processes. We are offering extra credit points in your class based on your participation. For this 30 minute process, your instructor has agreed to award points of extra credit to your course grade should you choose to participate. Participation is voluntary. Please visit the following website to obtain more information about participation.

www.pdx.edu/websurveyor/surveyXXX $<$ LINK ADVANCES TO INFORMED CONSENT (Appendix C)> 


\title{
APPENDIX C
}

\author{
Informed Consent
}

Dear Potential Participant,

The following study has been designed to capture your reactions to the job selection process. You will be asked to complete a short Internet-based application and employment test. Following the test, you will be asked to answer questions about the testing process. Participation in this study should take no longer than 30 minutes of your time.

Your participation is voluntary and confidential. You do not have to take part in this study, and participation is voluntary. Your participation will not affect your standing as a student at Portland State University. You may discontinue participation in the study at any time. If you feel uncomfortable answering any question, you may skip it. Your name will be kept confidential. Your individual responses will be kept confidential.

If you have any concerns or questions about this study, please contact the Chair of the Human Subjects Research Review Committee, Office of Research and Sponsored Projects, 111 Cramer Hall, Portland State University, Portland, Oregon, 97207, (503) 725-8182. You may also contact Clayton A. Yonce (407) 446-4606 or cyonce@pdx.edu.

If you would like to participate in this survey please click "Yes". If not, simply close this survey on your screen. By clicking "Yes" you state that you agree to and understand the terms and conditions stated above.

I agree to participate: $<$ BUTTON ADVANCES TO NEXT PAGE $>$ 


\section{APPENDIX D}

\section{Letter to Participant}

\section{Dear Participant,}

Thank you for agreeing to participate in this study. Your participation is voluntary, and you may cease participation at any time by closing the webpage.

Please imagine that you are applying for the job of "Business Associate" with a retail store called "The Smith Company." The Smith Company, a well-known retailer that sells a variety of products (including clothing, electronics, and housewares), is rated positively by employees for being a good place to work.

In order to select applicants for positions, The Smith Company uses an Internet-based job application and test.. As you imagine that you are applying for a job with The Smith Company, please be aware that the participant who is selected for the position to which you are applying with The Smith Company will be awarded 100 dollars, so you are encouraged to do your best when completing the Internet-based job application and test.

After clicking NEXT, you will proceed to the Internet-based application and test. Please complete the application and test. You will then be asked to answer questions regarding your experience.

$<$ BUTTON ADVANCES TO NEXT PAGE $>$ 


\section{APPENDIX E \\ Employment Application and Test}

Note. This application is based on the Internet-Based Best Buy Application

Welcome to The Smith Company's employment website. The Smith Company is currently seeking applicants for the position of Business Associate. Please complete the following application and test with care, as these materials will be used to determine whether or not you will be hired by The Smith Company.

Business Associate. You will use your education and experience to ensure that The Smith Company meets its yearly goals related to providing customers with the products they need. In this role, you will have the opportunity to work with members of the sales team to determine what products and services are most desired by our customers. You will then make recommendations to management.

The application process will take approximately 10 to 50 minutes depending on the position you are applying for. Please have your employment history, as well as your personal information, readily available before continuing with this application.

\section{APPLICATION FOR: Sales Associate}

\section{$<$ BUTTON ADVANCE TO NEXT PAGE $>$}

Giving false information of any kind may result in refusal of employment or dismissal.

Applicants with disabilities may be entitled to reasonable accommodation under the terms of the Americans with Disabilities Act and certain state or local laws. A reasonable accommodation is a change in the way things are normally done which will ensure an equal employment opportunity without imposing undue hardship on The Smith Company. Please inform a company management representative if you need assistance completing any forms or to otherwise participate in the application process.

Your completed application will remain active for 45 days.

\section{$<$ BUTTON ADVANCES TO NEXT PAGE $>$}

The Smith Company is committed to a policy of Equal Employment Opportunity (EEO) and maintains an Affirmative Action program. The Smith Company will not discriminate on the basis of age, sex, race, color, creed, religion, ethnicity, sexual orientation, gender 
identity, gender expression, national origin, citizenship, disability, marital status, military or veteran status, or any other legally recognized protected basis under federal, state, or local laws, regulations or ordinances. Our management is dedicated to ensuring the fulfillment of this policy. When necessary, we will reasonably accommodate employees and applicants with disabilities and with religious requirements necessitating accommodation.

The Smith Company is subject to the Worker's Compensation laws of the state of Rhode Island.

By proceeding you are indicating that you have carefully read and fully understand the preceding statements.

$<$ BUTTON ADVANCES TO NEXT PAGE $>$

Applicant page with Explanation Condition (Appendix F) or No Explanation Condition (Appendix G)

$<$ BUTTON ADVANCES TO NEXT PAGE $>$

Personal Identification

IT IS VERY IMPORTANT that you accurately enter the last four digits of your Social Security Number. . You must have a valid Social Security Number to apply for employment at The Smith Company.

Note: Bolded items are required.

- Last Four Digits of Your Social Security Number

$<$ BUTTON ADVANCES TO NEXT PAGE $>$

Personal Information

- Last Name:

- Legal First Name:

- Suffix (ex. Jr, Sr, III):

- Preferred Name:

- Middle Name (leave blank if you do not have a middle name):

$<$ BUTTON ADVANCES TO NEXT PAGE $>$

Contact Information \& Address 
- Home Phone \#:

- Work Phone \#:

- Alternate Phone \#:

- Email Address:

- Mailing Address:

- Apt.:

- City:

- State or Province:

- County:

$<$ BUTTON ADVANCES TO NEXT PAGE $>$

\section{Background Check(s)}

Thank you for your interest in working at The Smith Company. As a part of the hiring process, we require that a Background Check be performed on all applicants who are selected for positions and receive job offers. Therefore, if you are offered a position following this application process, you may be asked to complete authorizations to consent to several types of background checks.

Types of checks which may be performed, and for which we may request your authorizations, include criminal history information reports, private companies' dishonesty, drug offense or violence reports, or credit bureau reports or motor vehicle reports as authorized under the Fair Credit Reporting Act ("FCRA").

If offered a position, would you be willing to submit to a background check, to include a criminal background check?

- Yes

- No

\section{$<$ BUTTON ADVANCES TO NEXT PAGE $>$}

\section{Pre-Criminal Background Check}

Note - The existence of a criminal history will not automatically disqualify you from the job you are applying for.

Have you been convicted of a felony in the past 10 years?

Do not answer "Yes" if your conviction record has been annulled, expunged, or sealed.

- Yes

- No

Have you been convicted of any other crimes in the past 10 years? 
- Yes

- No

$<$ BUTTON ADVANCES TO NEXT PAGE $>$

\section{Business Associate Job Description}

As a Business Associate for The Smith Company, you will use your education and experience to ensure that The Smith Company meets its yearly goals related to providing customers with the products they need. In this role, you will have the opportunity to work with members of the sales team to determine what products and services are most desired by our customers. You will then make recommendations to management. You must be able to work at least 20 hours per work week.

Preferred qualifications:

- Dedicated and reliable

- Some college and/or professional training

Knowledge of psychology, general business, and/or sales

Are you able to perform these essential functions with or without reasonable accommodation?

- Yes

- No

Can you provide documentation establishing your identity and eligibility to be legally employed?

- Yes

- No

$<$ BUTTON ADVANCES TO NEXT PAGE $>$

\section{Work Hours Availability}

The Smith Company's full-time status is classified as a minimum of 32 hours per week, which will include day, evening and weekend hours. The following are the schedules we offer: Full-Time, Part-Time Weekdays, Part-Time Weekends, and Part-Time Flexible, which includes days, evenings and weekends. Please select the schedule which you prefer.

Desired Schedule:

- Full Time

- Part-Time Weekdays 
- Part-Time Weekends

- Part-Time Flexible

- Seasonal/Occasional

$<$ BUTTON ADVANCES TO NEXT PAGE $>$

\section{Voluntary Information}

The following questions are completely voluntary. Refusal to answer questions will not disqualify you from opportunities at The Smith Company. Your answers will not be considered during the hiring process.

What is your gender?

- Male

- Female

What is your Ethnic Background?

- American Indian or Alaska Native (Not Hispanic or Latino)

- Asian (Not Hispanic or Latino)

- Black or African American (Not Hispanic or Latino)

- Hispanic or Latino

- Native Hawaiian or Other Pacific Islander (Not Hispanic or Latino)

- Two or More Races (Not Hispanic or Latino)

- White (Not Hispanic or Latino)

This completes the voluntary question section.

$<$ BUTTON ADVANCES TO NEXT PAGE $>$

Work History

Have you ever been terminated due to attendance problems?

- Yes

- No

Have you ever been formally disciplined for performance problems by an employer?

- Yes

- No

How many jobs have you had in the past 3 years?

- 1

- $2-3$

- $4-5$ 
- 6 or more

Please enter your most recent employer for the employment history information section of this application.

Most Recent Employer

- Company Name:

- City:

- State:

- Phone \#:

What was your starting job title?

- Open Text Box

What was your starting hourly wage?

- Open Text Box

List your major responsibilities at this job:

- Open Text Box

Month \& year when you started (ex: 12/2000):

- Open Text Box

If you are still employed by this employer, what is your current job title?

- Open Text Box

What is your current hourly wage?

- Open Text Box

What is your supervisor's name?

- Last Name:

- First Name:

May we contact this employer?

- Yes

- No

$<$ BUTTON ADVANCES TO NEXT PAGE $>$

Education

Please select the highest education attained or in-progress: 
- GED/High School Diploma

- Trade School

- In-Progress University/College Undergraduate Degree

- Associates Degree

- Bachelor's Degree

- Master's Degree

- Doctoral Degree

- Professional Degree

- Other

Please complete the following information for the highest level of schooling in-progress or completed:

- School Name:

- City:

- State/Province:

- Country:

How many years of college/university have you completed?

- Open Text Box

Have you graduated or received a college/university degree?

- Yes

- No

If so, please list your major or your primary course of study:

- Open Text Box

$<$ BUTTON ADVANCES TO NEXT PAGE $>$

References

Please list one personal or professional reference:

- Last Name:

- First Name:

- City:

- State:

- Relationship

- Occupation:

- Company Name:

$<$ BUTTON ADVANCES TO NEXT PAGE $>$ 
The following are statements about many attitudes and experiences. Read each statement and indicate whether you strongly disagree, disagree, agree, or strongly agree with the statement. Work quickly - choose the answer that comes to mind first.

$<20$ personality items measuring aspects of conscientiousness $>$

$<$ BUTTON ADVANCES TO NEXT PAGE $>$ 


\section{APPENDIX F}

\section{Excuse Explanation Condition}

Every year, we receive many applications for positions with The Smith Company.

We use an Internet-based selection process that includes both an application and an assessment because it allows us to:

- Collect and use the information you provide to The Smith Company in order to select the best candidates

- Provide a secure environment so that your private information is protected.

- Comply with legal obligations related to employment

Cookies: This Internet-based application utilizes cookies to provide a better user experience for job applicants. These cookies are either removed when the user logs out or expire automatically after a specified time period.

IP addresses: This Internet-based application logs IP addresses to facilitate the diagnosis of potential server and/or problems with job applications.

$<$ BUTTON ADVANCES TO NEXT PAGE $>$ 


\section{APPENDIX G}

\section{Privacy Justification Explanation Condition}

Every year, we receive many applications for positions with The Smith Company.

We use an Internet-based selection process that includes both an application and an assessment. You can be confident in the safety of your personal information because:

- We have limited the amount of personal information we request from you.

- We continuously test our Internet-based selection website to ensure that personal information is safeguarded from unauthorized access.

- We have implemented technology and procedures to ensure that the information you provide is captured correctly.

- We do not sell, share, or disclose your personal information to third-parties.

Cookies: This Internet-based application utilizes cookies to provide a better user experience for job applicants. These cookies are either removed when the user logs out or expire automatically after a specified time period.

IP addresses: This Internet-based application logs IP addresses to facilitate the diagnosis of potential server and/or problems with job applications.

$<$ BUTTON ADVANCES TO NEXT PAGE $>$ 


\section{APPENDIX H}

\section{No Explanation Condition}

Every year, we receive many applications for positions with The Smith Company. In order to select employees, The Smith Company e utilizes an Internet-based selection process that includes both an application and an assessment.

Cookies: This Internet-based application utilizes cookies to provide a better user experience for job applicants. These cookies are either removed when the user logs out or expire automatically after a specified time period.

IP addresses: This Internet-based application logs IP addresses to facilitate the diagnosis of potential server and/or problems with job applications.

$<$ BUTTON ADVANCES TO NEXT PAGE $>$ 


\section{APPENDIX I}

\section{Control Over Information Condition}

You have completed the Internet-based job application and assessment for the position of Business Associate with The Smith Company.

Personal Information Release

You now have the option to either:

- Release your personal information (Name, Address, Social Security Number, etc.), application responses, and assessment results to the hiring manager at The Smith Company

or

- Release only application responses, assessment results, and phone number/email, without disclosing any personal information (Name, Address, Social Security Number, etc.)

Your decision will not affect the hiring decision of The Smith Company.

Please indicate your choice below by clicking the box that corresponds to your decision:

- I choose to release my personal information to the hiring manager at The Smith Company at this time.

o I wish to decline the release of my personal information to the hiring manager at The Smith Company at this time.

$<$ BUTTON ADVANCES TO NEXT PAGE $>$

Your application is now complete.

If your qualifications meet our requirements, and a job opening is available, a Hiring Manager will contact you. Your application will remain active for 45 days.

Thank you for your interest in employment with The Smith Company. 


\section{APPENDIX J}

\section{No Control Over Information Condition}

You have completed the Internet-based job application and assessment for the position of Business Associate with The Smith Company.

- Your personal information, application responses, and assessment result will now be forwarded to a hiring manager at The Smith Company.

If your qualifications meet our requirements, and a job opening is available, a Hiring Manager will contact you. Your application will remain active for 45 days.

Thank you for your interest in employment with The Smith Company. 
APPENDIX K

Participant Process

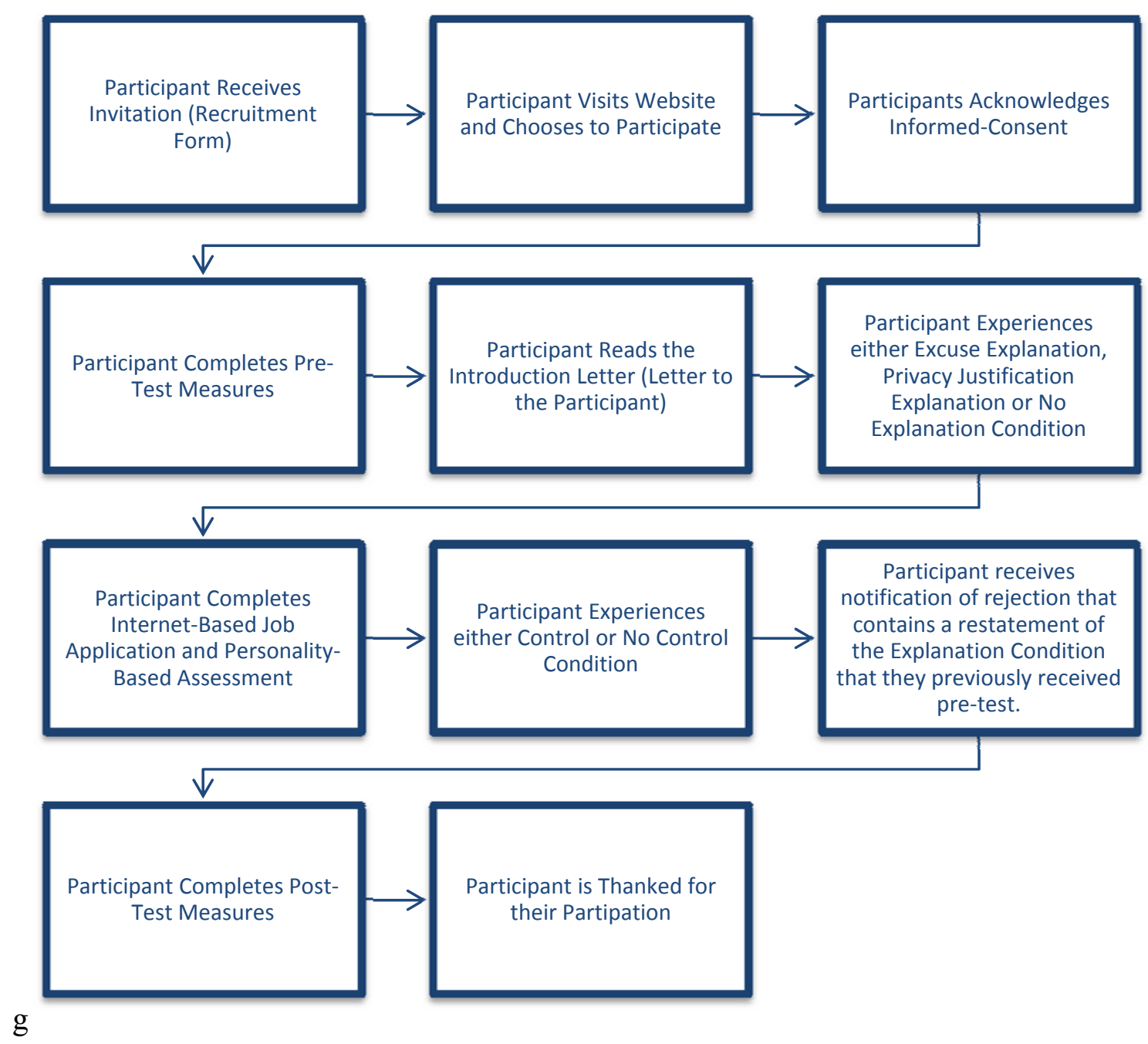




\section{APPENDIX L}

Decision Letter - Post-Test Excuse Explanation Condition

Dear $<$ NAME FROM APPLICATION $>$,

Thank you for completing the Internet-based job application and test for a position with The Smith Company.

Every year, we receive many applications for positions with The Smith Company.

We use an Internet-based selection process that includes both an application and an assessment because it allows us to:

- Collect and use the information you provide to The Smith Company in order to select the best candidates

- Provide a secure environment so that your private information is protected.

- Comply with legal obligations related to employment

The Smith Company has reviewed your responses to the Internet-based application and test. Unfortunately, we are unable to offer you a position at this time.

We wish you the best in your job search.

$<$ BUTTON ADVANCES TO NEXT PAGE $>$ 


\section{APPENDIX M}

\section{Decision Letter - Post-Test Privacy Justification Explanation Condition}

Dear $<$ NAME FROM APPLICATION $>$,

Thank you for completing the Internet-based job application and test for a position with The Smith Company.

Every year, we receive many applications for positions with The Smith Company.

We use an Internet-based selection process that includes both an application and an assessment. You can be confident in the safety of your personal information because:

- We have limited the amount of personal information we request from you.

- We continuously test our Internet-based selection website to ensure that personal information is safeguarded from unauthorized access.

- We have implemented technology and procedures to ensure that the information you provide is captured correctly.

- We do not sell, share, or disclose your personal information to third-parties.

The Smith Company has reviewed your responses to the Internet-based application and test. Unfortunately, we are unable to offer you a position at this time.

We wish you the best in your job search.

$<$ BUTTON ADVANCES TO NEXT PAGE $>$ 


\section{APPENDIX N}

\section{Decision Letter - Post-Test No Explanation Condition}

Dear $<$ NAME FROM APPLICATION $>$,

Thank you for completing the Internet-based job application and test for a position with The Smith Company.

The Smith Company has reviewed your responses to the Internet-based application and test. Unfortunately, we are unable to offer you a position at this time.

We wish you the best in your job search.

$<$ BUTTON ADVANCES TO NEXT PAGE $>$ 


\section{APPENDIX O}

\section{Thank You/Debrief Letter}

Dear Participant,

The study in which you participated was designed to capture your reactions to Internetbased selection procedures. Though every participant is declined a position with The Smith Company, a $\$ 100$ prize will be given to a random participant. You will be contacted via email if you are awarded the prize.

During your participation in this study, we collected personal information from you, including a portion of your social security number. This personal information is kept safe on a password-protected server and will be erased following the final download of the data from the websurvey program.

If you have any concerns or questions about this study, and/or you would like to receive the results of this study, please contact Clayton A. Yonce at (407) 446-4606 or cyonce@pdx.edu.

Thank you for your participation in this study. 


\section{APPENDIX P}

\section{Prize Award}

The $\$ 100$ prize offered in this study was awarded to a single participant who was randomly selected from a listing of eligible participants. Participants were eligible for the drawing if they completed one item in the pre-test section, one item in the fictitious Internet-based selection procedure, and one item in the post-test section. 